Supporting Information

\title{
Regiodivergent Cross-Dehydrogenative Coupling of Pyridines and Benzoxazoles: Discovery of Organic Halides as Regio-Switching Oxidants
}

\author{
Shuya Yamada, Kei Murakami, Kenichiro Itami \\ ${ }^{1}$ Institute of Transformative Bio-Molecules (WPI-ITbM) and Graduate School of Science, \\ Nagoya University, Chikusa, Nagoya 464-8602, Japan. \\ ${ }^{2}$ JST, ERATO, Itami Molecular Nanocarbon Project, Nagoya University, Chikusa, Nagoya \\ 464-8602, Japan \\ E-mail: itami@chem.nagoya-u.ac.jp
}

\section{Table of Contents}

$\begin{array}{ll}\text { 1. General } & \text { S2 }\end{array}$

2. Experimental Procedure $\quad$ S3

3. Optimization of C2-selective Reaction $\quad$ S4

4. Characterization Data for C3-selective Reaction S5-15

5. Characterization Data for C2-selective Reaction S16-18

6. ${ }^{1} \mathrm{H}$ and ${ }^{13} \mathrm{C}$ NMR Spectra $\quad$ S19-39

7. Validation of reduction of aryl/benzyl bromide $\quad$ S40-43 


\section{General}

Unless otherwise noted, all reactants or reagents including dry solvents were obtained from commercial suppliers and used as received. $\mathrm{Pd}(\mathrm{OAc})_{2}$, dehydrated pyridine, benzyl bromide, 1-bromo-2,4,6-triisopropylbenzene, 4-methylpyridine and benzoxazole were purchased from Wako chemicals. $\mathrm{Pd}(\mathrm{OPiv})_{2}$, 3-methylpyridine and 4-trifluoromethylpyridine were purchased from Sigma-Aldrich. 1,3-Dimesitylimidazolium chloride (IMes·HCl) and 3trifluoromethylpyridine were purchased from TCI. $\mathrm{CsOPiv}^{\mathrm{S} 1}$, substituted benzoxazoles ${ }^{\mathrm{S} 2}, \mathrm{~N}$ benzylpyridinium bromide ${ }^{\mathrm{S} 3}$ and benzyl pivalate ${ }^{\mathrm{S} 4}$ were synthesized according to procedures reported in the literature. Benzyl bromide was freshly purified by vacuum distillation before coupling reaction. Substituted pyridines were used as received. All reactions were performed with dry solvents under an atmosphere of $\mathrm{N}_{2}$ gas in flame-dried glassware using standard vacuum-line techniques. All work-up and purification procedures were carried out with reagent-grade solvents in air.

Analytical thin-layer chromatography (TLC) was performed using E. Merck silica gel 60 F254 precoated plates $(0.25 \mathrm{~mm})$. The developed chromatogram was analyzed by UV lamp $(254 \mathrm{~nm})$ or phosphomolybdic acid/sulfuric acid solution. Flash column chromatography was performed with E. Merck silica gel 60 (230-400 mesh). Silica-gel column chromatography was performed on an Isolera Spektra instrument equipped with a Biotage SNAP Ultra $10 \mathrm{~g}$ cartridge for $0.25 \mathrm{mmol}$ scale reactions. Preparative recycling gel permeation chromatography (GPC) was performed with a JAI LC-9260 II NEXT instrument equipped with JAIGEL1H/JAIGEL-2H columns using chloroform as an eluent. Nuclear magnetic resonance (NMR) spectra were recorded on a JEOL JNM-ECA-600 $\left({ }^{1} \mathrm{H} 600 \mathrm{MHz},{ }^{13} \mathrm{C} 150 \mathrm{MHz}\right)$ spectrometer. Chemical shifts for ${ }^{1} \mathrm{H}$ NMR are expressed in parts per million (ppm) relative to tetramethylsilane $(\delta 0.00 \mathrm{ppm})$. Chemical shifts for ${ }^{13} \mathrm{C}$ NMR are expressed in ppm relative to $\mathrm{CDCl}_{3}(\delta 77.2 \mathrm{ppm})$. Data are reported as follows: chemical shift, multiplicity ( $\mathrm{s}=$ singlet, $\mathrm{d}=$ doublet, $\mathrm{dd}=$ doublet of doublets, $\mathrm{t}=$ triplet, $\mathrm{dt}=$ doublet of triplets, $\mathrm{q}=$ quartet, $\mathrm{m}=$ multiplet), coupling constant $(\mathrm{Hz})$, and integration.

\footnotetext{
${ }^{\mathrm{S} 1}$ Campo, M.; Larock, R. Org. Lett. 2000, 2, 3675-3677.

${ }^{\text {S2 }}$ Guo, S.; Qian, B.; Xie, Y.; Xia, C.; Huang, H. Org. Lett. 2011, 13, 522-525.

${ }^{53}$ Peng, W.; Zhu, S. J. Chem. Soc. Perkin Trans. 1 2001, 3204-3210.

${ }^{\mathrm{s} 4}$ Bender, D.; Peterson, J.; McCarthy, J.; Gunaydin, H.; Takano, Y.; Houk, K. Org. Lett. 2008, 10, 509-511.
} 


\section{Experimental Procedure}

\section{C3-selective Reaction}

The reaction in Figure 2a is representative. A $20-\mathrm{mL}$ Schlenk tube equipped with J. Young O-ring tap containing a magnetic stirring bar was dried with a heat gun under reduced pressure and filled with nitrogen gas after cooling to room temperature. To this vessel was added $\mathrm{Pd}(\mathrm{OAc})_{2}(5.6 \mathrm{mg}, 0.025 \mathrm{mmol})$, and then the tube was introduced into an argonatmosphere glovebox. To the reaction tube was added CsOPiv (176 mg, $0.50 \mathrm{mmol})$ and the tube was taken out from the glovebox. To the tube were added benzoxazole $(29.8 \mathrm{mg}, 0.25$ mmol), 1-bromo-2,4,6-triisopropylbenzene (127 $\mu \mathrm{L}, 0.50 \mathrm{mmol})$ and dry pyridine $(1.5 \mathrm{~mL})$ under a stream of nitrogen gas. The vessel was sealed with O-ring tap and then stirred at $170{ }^{\circ} \mathrm{C}$ for $17 \mathrm{~h}$ in an 8-well reaction heat block. After cooling to room temperature, the reaction mixture was passed through a short pad of Celite (EtOAc was used as an eluent.) The filtrate was concentrated in vacuo. After removal of pyridine, the mixture was purified through silica-gel column chromatography by Isolera Spektra instrument equipped with a Biotage SNAP Ultra $10 \mathrm{~g}$ cartridge $(n$-hexane/EtOAc $=19: 1$ to $3: 1)$ to give 1a $(29.0 \mathrm{mg})$ in $59 \%$ yield $(\mathrm{C} 2 / \mathrm{C} 3 / \mathrm{C} 4=17: 74: 9)$.

\section{C2-selective Reaction}

The reaction of pyridine with benzoxazole in Figure 4 is representative. A $20-\mathrm{mL}$ Schlenk tube equipped with $\mathrm{J}_{\text {. Young }}{ }^{\mathrm{O}} \mathrm{O}$-ring tap containing a magnetic stirring bar was dried with a heat gun under reduced pressure and filled with nitrogen gas after cooling to room temperature. To this vessel were added $\mathrm{Pd}(\mathrm{OPiv})_{2}(7.7 \mathrm{mg}, 0.025 \mathrm{mmol})$ and $\mathrm{IMes} \cdot \mathrm{HCl}(8.5$ $\mathrm{mg}, 0.025 \mathrm{mmol}$ ), and then the tube was introduced into an argon-atmosphere glovebox. To the reaction tube was added $\mathrm{CsOPiv}(176 \mathrm{mg}, 0.50 \mathrm{mmol})$ and the tube was taken out from the glovebox. To the tube were added benzoxazole $(29.8 \mathrm{mg}, 0.25 \mathrm{mmol})$, distilled benzyl bromide $(59 \mu \mathrm{L}, 0.50 \mathrm{mmol})$ and dry pyridine $(1.5 \mathrm{~mL})$ under a stream of nitrogen gas. The vessel was sealed with O-ring tap and then stirred at $170{ }^{\circ} \mathrm{C}$ for $17 \mathrm{~h}$ in an 8 -well reaction block. After cooling to room temperature, the reaction mixture was passed through a short pad of Celite ${ }^{\bullet}$ (EtOAc was used as an eluent). The filtrate was concentrated in vacuo. After removal of pyridine, the mixture was purified through silica-gel column chromatography by Isolera Spektra instrument equipped with a Biotage SNAP Ultra $10 \mathrm{~g}$ cartridge (nhexane/EtOAc $=19: 1$ to $3: 1)$ to give $\mathbf{2 a}(36 \mathrm{mg})$ in $74 \%$ yield . 


\section{Optimization of C2-selective Reaction}

Ld source (10 mol\%)
Ligand (10 mol\%)
$\mathrm{PhCH}_{2} \mathrm{Br}(2$ equiv)
$\mathrm{CsOPiv}(3$ equiv)




\section{Characterization Data for C3-Selective Reaction}<smiles>c1cncc(-c2nc3ccccc3o2)c1</smiles>

1a<smiles>c1cncc(-c2nc3ccccc3o2)c1</smiles>

1a-C3 Major isomer<smiles>c1ccc(-c2nc3ccccc3o2)nc1</smiles>

2a<smiles>c1ccc2oc(-c3ccncc3)nc2c1</smiles>

1a-C4

The product 1a (Figure 2b, $33.1 \mathrm{mg}$, light yellow solid) was obtained as a mixture of regioisomers (1a-C3, 2a, and 1a-C4), which were inseparable with silica-gel column chromatography or gel permeation chromatography. The ratio of regioisomers was determined by ${ }^{1} \mathrm{H}$ NMR spectroscopy.

2-(Pyridin-3-yl)benzo[d]oxazole (1a-C3): 1a-C3 is known compound and showed the identical ${ }^{1} \mathrm{H}$ NMR spectra according to the literature. ${ }^{\mathrm{S5}}$

2-(Pyridin-2-yl)benzo[d]oxazole (2a): 2a is known compound and showed the identical ${ }^{1} \mathrm{H}$ NMR spectra according to the literature. ${ }^{\mathrm{S} 5}$

2-(Pyridin-4-yl)benzo[d]oxazole (1a-C4): 1a-C4 is known compound and showed the identical ${ }^{1} \mathrm{H}$ NMR spectra according to the literature. ${ }^{\mathrm{S} 5}$

${ }^{\text {s5 }}$ Amaike, K.; Muto, K.; Yamaguchi, J.; Itami, K. J. Am. Chem. Soc. 2012, 134, 13573 13576. 


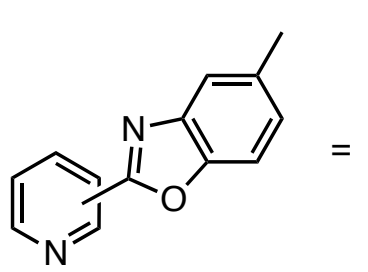

$1 b$<smiles>Cc1ccc2oc(-c3cccnc3)nc2c1</smiles>

1b-C3 Major isomer<smiles>Cc1ccc2oc(-c3ccccn3)nc2c1</smiles>

1b-C2<smiles>Cc1ccc2oc(-c3ccncc3)nc2c1</smiles>

1b-C4

The product 1b (Figure 3, $34.0 \mathrm{mg}$, light yellow solid) was obtained as a mixture of regioisomers (1b-C3, 1b-C2, and 1b-C4), which were inseparable with silica-gel column chromatography or gel permeation chromatography. The ratio of regioisomers was determined by ${ }^{1} \mathrm{H}$ NMR spectroscopy.

5-Methyl-2-(pyridin-3-yl)benzo[d] oxazole (1b-C3): 1b-C3 is known compound and showed the identical ${ }^{1} \mathrm{H}$ NMR spectra according to the literature. ${ }^{\mathrm{S} 6}$

5-Methyl-2-(pyridin-2-yl)benzo[d]oxazole (1b-C2): 1b-C2 is known compound and showed the identical ${ }^{1} \mathrm{H}$ NMR spectra according to the literature. ${ }^{\mathrm{s} 6}$

5-Methyl-2-(pyridin-4-yl)benzo[d]oxazole (1b-C4): The ${ }^{1} \mathrm{H}$ NMR data was assigned by analogy to compound 1a-C4. ${ }^{1} \mathrm{H}$ NMR $\left(\mathrm{CDCl}_{3}\right) \delta 2.49$ (s, 3H), 7.19-7.22 (m, 1H), 7.44-7.49 (m, 1H), 7.57-7.60 (m, 1H), $8.06(\mathrm{~d}, J=1.2 \mathrm{~Hz}, 2 \mathrm{H}), 8.81(\mathrm{~s}, 2 \mathrm{H})$.

HR-MS and ${ }^{13} \mathrm{C}$ NMR data for the mixture of $\mathbf{1 b}-\mathbf{C 3}, \mathbf{1 b}-\mathbf{C 2}$, and 1b-C4: ${ }^{13} \mathrm{C} \mathrm{NMR}\left(\mathrm{CDCl}_{3}\right) \delta$ $21.70,110.29,110.46,110.75,120.32,120.63,121.12,123.52,123.81,123.88,125.59$, $127.04,127.70,134.66,134.80,134.99,137.21,142.18,146.61,148.91,149.20,149.51$, 150.45, 150.87, 152.08, 160.93, 161.71; HR-MS (ESI-MS, positive): $\mathrm{m} / \mathrm{z}=211.0862 \mathrm{calcd}$ for $\mathrm{C}_{13} \mathrm{H}_{11} \mathrm{~N}_{2} \mathrm{O}: 211.08663[M+\mathrm{H}]^{+}$.

\footnotetext{
${ }^{56}$ Shen, X.-B.; Zhang, Y.; Chen, W.-X.; Xiao, Z.-K.; Hu, T.-T.; Shao, L.-X. Org. Lett. 2014, $16,1984-1987$.
} 


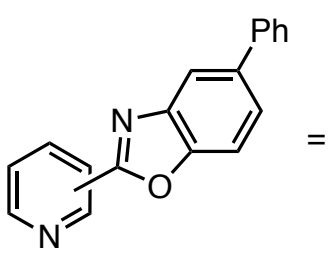

$1 c$

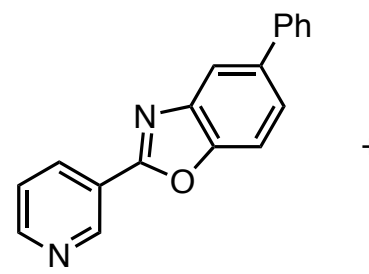

1c-C3 Major isomer
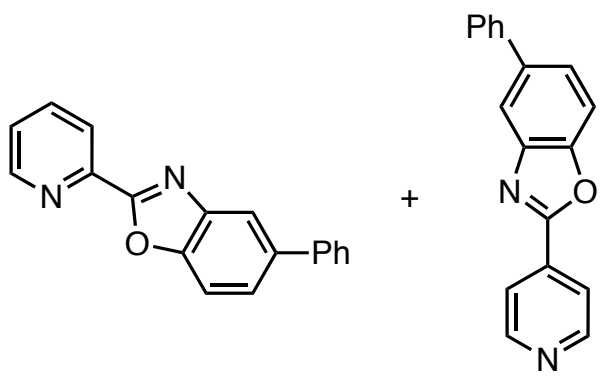

$2 b$

1c-C4

The product 1c (Figure 3, $36.0 \mathrm{mg}$, light yellow solid) was obtained as a mixture of regioisomers (1c-C3, 2b, and 1c-C4), which were inseparable with silica-gel column chromatography or gel permeation chromatography. The ratio of regioisomers was determined by ${ }^{1} \mathrm{H}$ NMR spectroscopy.

5-Phenyl-2-(pyridin-3-yl)benzo[d]oxazole (1c-C3): The ${ }^{1} \mathrm{H}$ NMR data was assigned by analogy to compound 1a. ${ }^{1} \mathrm{H}$ NMR $\left(\mathrm{CDCl}_{3}\right) \delta 7.39(\mathrm{t}, J=7.2 \mathrm{~Hz}, 1 \mathrm{H}), \delta 7.47-7.50(\mathrm{~m}, 3 \mathrm{H})$, 7.62-7.68 (m, 4H), 7.99-8.02 (m, 1H), $8.54(\mathrm{dt}, J=7.2,2.4 \mathrm{~Hz}, 1 \mathrm{H}), 8.78(\mathrm{dd}, J=4.8,1.8$ $\mathrm{Hz}, 1 \mathrm{H}), 9.50(\mathrm{~d}, J=1.2 \mathrm{~Hz}, 1 \mathrm{H})$.

5-Phenyl-2-(pyridin-2-yl)benzo[ $[\boldsymbol{d}]$ oxazole (2b): The compound data was determined from the pure form of $\mathbf{2 b}$, which was synthesized through the C2-selective reaction. ${ }^{1} \mathrm{H}$ NMR $\left(\mathrm{CDCl}_{3}\right) \delta 7.37-7.39(\mathrm{~m}, 1 \mathrm{H}), 7.46-7.49(\mathrm{~m}, 3 \mathrm{H}), 7.64(\mathrm{~d}, J=7.8 \mathrm{~Hz}, 3 \mathrm{H}), 7.71(\mathrm{~d}, J=8.4 \mathrm{~Hz}$, 1H), $7.89(\mathrm{t}, J=1.8 \mathrm{~Hz}, 1 \mathrm{H}), 8.02(\mathrm{~s}, 1 \mathrm{H}), 8.38(\mathrm{~d}, J=7.2 \mathrm{~Hz}, 1 \mathrm{H}), 8.83(\mathrm{~d}, J=4.2 \mathrm{~Hz}, 1 \mathrm{H})$; ${ }^{13} \mathrm{C} \mathrm{NMR}\left(\mathrm{CDCl}_{3}\right) \delta 111.38,119.16,123.68,125.78,125.89,127.52,127.64,129.05,137.27$, 139.04, 141.05, 142.57, 146.17, 150.48, 150.76, 162.19; HR-MS (ESI-MS, positive): $\mathrm{m} / \mathrm{z}=$ 273.1013. calcd for $\mathrm{C}_{18} \mathrm{H}_{13} \mathrm{~N}_{2} \mathrm{O}: 273.1022[M+\mathrm{H}]^{+}$

5-Phenyl-2-(pyridin-4-yl)benzo[d]oxazole (1c-C4): The ${ }^{1} \mathrm{H}$ NMR data was assigned by analogy to compound 1a-C4. ${ }^{1} \mathrm{H}$ NMR $\left(\mathrm{CDCl}_{3}\right) \delta 7.39(\mathrm{t}, J=7.2 \mathrm{~Hz}, 1 \mathrm{H}), 7.47-7.50(\mathrm{~m}, 3 \mathrm{H})$, 7.62-7.68 (m, 3H), 7.99-8.02 (m, 1H), $8.11(\mathrm{~d}, J=5.4 \mathrm{~Hz}, 2 \mathrm{H}), 8.84(\mathrm{~d}, J=5.4 \mathrm{~Hz}, 2 \mathrm{H})$.

${ }^{13} \mathrm{C}$ NMR data for the mixture of $\mathbf{1 c - C 3}, \mathbf{2 b}$, and 1c-C4: ${ }^{13} \mathrm{C} \mathrm{NMR}\left(\mathrm{CDCl}_{3}\right) \delta 110.94,111.11$, $111.39,118.84,119.19,121.18,123.67,123.69$, 123.86, 125.51, 125.78, 125.89, 126.13, $127.54,127.58,127.62,127.66,128.90,129.09$, 134.42, 134.90, 137.26, 139.02, 139.24, $140.86,141.00,141.07,142.62,146.21,149.00,150.48,150.50,150.55,150.78,150.94$, 150.30, 161.37, 161.47. HR-MS (ESI-MS, positive): $m / z=273.1016$. calcd for $\mathrm{C}_{18} \mathrm{H}_{13} \mathrm{~N}_{2} \mathrm{O}$ : $273.1022[M+\mathrm{H}]^{+}$. 
<smiles>CC(C)(C)c1ccc2oc(-c3cccnc3)nc2c1</smiles>

1d<smiles>CC(C)(C)c1ccc2oc(-c3cccnc3)nc2c1</smiles>

1d-C3 Major isomer<smiles>CC(C)(C)c1ccc2oc(-c3ccccn3)nc2c1</smiles><smiles>CC(C)(C)c1ccc2oc(-c3ccncc3)nc2c1</smiles>

2c

The product 1d (Figure 3, $46.7 \mathrm{mg}$, light yellow solid) was obtained as a mixture of regioisomers (1d-C3, 2c, and 1d-C4), which were inseparable with silica-gel column chromatography or gel-permeation chromatography. The ratio of regioisomers was determined by ${ }^{1} \mathrm{H}$ NMR spectroscopy.

5-(tert-Butyl)-2-(pyridin-3-yl)benzo[d]oxazole (1d-C3): 1d-C3 is known compound and showed the identical ${ }^{1} \mathrm{H}$ NMR spectra according to the literature. ${ }^{\mathrm{S} 6}$

5-(tert-Butyl)-2-(pyridin-2-yl)benzo[d]oxazole (2c): 2c is known compound and showed the identical ${ }^{1} \mathrm{H}$ NMR spectra according to the literature. ${ }^{\mathrm{S} 6}$

5-(tert-Butyl)-2-(pyridin-4-yl)benzo[d] $]$ oxazole (1d-C4): The ${ }^{1} \mathrm{H}$ NMR data was assigned by analogy to compound 1a-C4. ${ }^{1} \mathrm{H}$ NMR $\left(\mathrm{CDCl}_{3}\right) \delta 1.41$ (s, 9H), 7.43-7.49 (m, 2H), 7.82-7.84 (m, 1H), 8.06-8.08 (m, 2H), $8.81(\mathrm{~d}, J=2.4 \mathrm{~Hz}, 2 \mathrm{H})$.

HR-MS and ${ }^{13} \mathrm{C}$ NMR data for the mixture of 1d-C2, 2c, and 1d-C4: ${ }^{13} \mathrm{C} \mathrm{NMR}\left(\mathrm{CDCl}_{3}\right) \delta$ $31.88,31.94,35.19,110.09,110.28,110.57,116.94,117.18,117.25,121.11,123.53,123.72$, $123.87,123.93,124.13,124.40,125.60,134.71$, 134.79, 137.25, 141.94, 146.47, 148.76, 148.92, 149.00, 149.32, 150.44, 150.91, 152.09, 160.91, 160.99, 161.78; HR-MS (ESI-MS, positive): $m / z=253.1330$. calcd for $\mathrm{C}_{16} \mathrm{H}_{17} \mathrm{~N}_{2} \mathrm{O}: 253.1335[M+\mathrm{H}]^{+}$. 


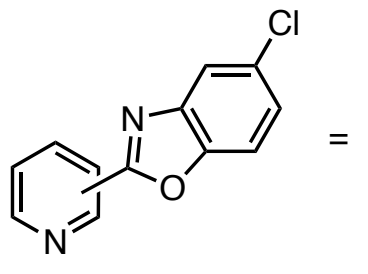

$1 e$<smiles>Clc1ccc2oc(-c3cccnc3)nc2c1</smiles>
1e-C3
Major isomer<smiles>Clc1ccc2oc(-c3ccccn3)nc2c1</smiles><smiles>Clc1ccc2oc(-c3ccncc3)nc2c1</smiles>

1e-C2 1e-C4

The product 1e (Figure 3, $24.1 \mathrm{mg}$, white solid) was obtained as a mixture of regioisomers (1e-C3, 1e-C2, and 1e-C4), which were inseparable with silica-gel column chromatography or gel-permeation chromatography. The ratio of regioisomers was determined by ${ }^{1} \mathrm{H}$ NMR spectroscopy.

5-(Chloro)-2-(pyridin-3-yl)benzo[d]oxazole (1e-C3): 1e-C3 is known compound and showed the identical ${ }^{1} \mathrm{H}$ NMR spectra according to the literature. ${ }^{\mathrm{S} 7}$

5-(Chloro)-2-(pyridin-2-yl)benzo[d]oxazole (1e-C2): The ${ }^{1} \mathrm{H}$ NMR data was assigned by analogy to compound 2c. ${ }^{1} \mathrm{H}$ NMR $\left(\mathrm{CDCl}_{3}\right) \delta$ 7.35-7.40 (m, 1H), 7.46-7.48 (m, 1H), 7.58 (dd, $J=8.4 \mathrm{~Hz}, J=1.8 \mathrm{~Hz}, 1 \mathrm{H}), 7.76-7.80(\mathrm{~m}, 1 \mathrm{H}), 7.90(\mathrm{t}, J=7.8 \mathrm{~Hz}, 1 \mathrm{H}), 8.34(\mathrm{~d}, J=7.8 \mathrm{~Hz}$, $1 \mathrm{H}), 8.82(\mathrm{~d}, J=2.4 \mathrm{~Hz}, 1 \mathrm{H})$.

5-(Chloro)-2-(pyridin-4-yl)benzo[d] oxazole (1e-C4): The ${ }^{1} \mathrm{H}$ NMR data was assigned by analogy to compound 1a-C4. ${ }^{1} \mathrm{H}$ NMR $\left(\mathrm{CDCl}_{3}\right) \delta$ 7.35-7.40 (m, 1H), 7.46-7.48 (m, 1H), 7.76$7.80(\mathrm{~m}, 1 \mathrm{H}), 8.05(\mathrm{t}, J=4.8 \mathrm{~Hz}, 2 \mathrm{H}), 8.82(\mathrm{~d}, J=2.4 \mathrm{~Hz}, 2 \mathrm{H})$.

HR-MS and ${ }^{13} \mathrm{C}$ NMR data for the mixture of 1e-C3, 1e-C2, and 1e-C4: ${ }^{13} \mathrm{C} \mathrm{NMR}\left(\mathrm{CDCl}_{3}\right) \delta$ 110.93 , 111.39, 111.66, 111.85, 112.11, 120.42, 120.73, 120.78, 121.20, 123.88, 125.081, $126.04,126.18,126.54,126.81,130.62$, 130.84, 134.02, 134.86, 135.00, 137.23, 137.31, $141.96,143.00,143.06,143.08,145.82,148.96$, 149.05, 149.50, 150.56, 150.98, 152.22, 152.57, 160.86, 162.08, 162.19, 162.85; HR-MS (ESI-MS, positive): $\mathrm{m} / z=231.0316$. calcd for $\mathrm{C}_{12} \mathrm{H}_{8} \mathrm{ClN}_{2} \mathrm{O}: 231.0320[M+\mathrm{H}]^{+}$.

\footnotetext{
${ }^{7}$ Zhang, W.; Tian, Y.; Zhao, N.; Wang, Y.; Li, J.; Wang, Z. Tetrahedron 2014, 70, 6120_ 6126.
} 
<smiles>Cc1ccncc1-c1nc2ccccc2o1</smiles>

$1 f$<smiles>Cc1ccncc1-c1nc2ccccc2o1</smiles>

1f-C3 Major isomer<smiles>Cc1ccnc(-c2nc3ccccc3o2)c1</smiles>

1f-C2

The product $\mathbf{1 f}$ (Figure 3, $31.4 \mathrm{mg}$, white solid) was obtained as a mixture of regioisomers (1f-C3 and 1f-C2), which were inseparable with silica-gel column chromatography or gel permeation chromatography. The ratio of regioisomers was determined by ${ }^{1} \mathrm{H}$ NMR spectroscopy.

2-(4-Methylpyridin-3-yl)benzo[d] oxazole (1f-C3): ${ }^{1} \mathrm{H}$ NMR $\left(\mathrm{CDCl}_{3}\right) \delta 2.83$ (s, 3H), 7.27 (d, $J=5.4 \mathrm{~Hz}, 1 \mathrm{H}), 7.38-7.40(\mathrm{~m}, 2 \mathrm{H}), 7.61(\mathrm{~d}, J=7.2 \mathrm{~Hz}, 1 \mathrm{H}), 7.80-7.82(\mathrm{~m}, 2 \mathrm{H}), 8.57$ (d, $J=$ $5.4 \mathrm{~Hz}, 1 \mathrm{H}), 9.44(\mathrm{~s}, 1 \mathrm{H})$.

2-(4-Methylpyridin-2-yl)benzo[d]oxazole (1f-C2): The authentic product was synthesized with the reported procedure. ${ }^{\mathrm{s} 8}{ }^{1} \mathrm{H}$ NMR $\left(\mathrm{CDCl}_{3}\right) \delta 2.48(\mathrm{~s}, 3 \mathrm{H}), 7.27$ (s, 1H), 7.38-7.42 (m, 2H), $7.67(\mathrm{dd}, J=7.2,1.8 \mathrm{~Hz}, 1 \mathrm{H}), 7.83(\mathrm{dd}, J=6.6,2.4 \mathrm{~Hz}, 1 \mathrm{H}), 8.22$ (s, 1H), 8.67 (d, $J=$ $4.8 \mathrm{~Hz}, 1 \mathrm{H}) ;{ }^{13} \mathrm{C} \mathrm{NMR}\left(\mathrm{CDCl}_{3}\right) \delta 21.17,111.32,120.64,124.39,124.98,126.03,126.64$, 141.87, 145.98, 148.60, 150.15, 151.16, 161.80; HR-MS (ESI-MS, positive): $m / z=211.0864$. calcd for $\mathrm{C}_{13} \mathrm{H}_{11} \mathrm{~N}_{2} \mathrm{O}: 211.0866[M+\mathrm{H}]^{+}$.

HR-MS and ${ }^{13} \mathrm{C}$ NMR data for the mixture of 1f-C3 and 1f-C2: ${ }^{13} \mathrm{C} \mathrm{NMR}\left(\mathrm{CDCl}_{3}\right) \delta$ 21.22, $21.83,110.82,111.36,120.49,120.69,123.15,124.44,124.83,125.03,125.74,126.08$, $126.47,126.67,141.93,141.99,146.04,141.71,148.64,150.21,150.36,150.63,151.18$, 161.17, 161.86; HR-MS (ESI-MS, positive): $m / z=211.0862$. calcd for $\mathrm{C}_{13} \mathrm{H}_{11} \mathrm{~N}_{2} \mathrm{O}: 211.0866$ $[M+\mathrm{H}]^{+}$.

${ }^{\text {s8 }}$ Yan, X.-M.; Mao, X.-R.; Huang, Z.-Z. Heterocycles 2011, 83, 1371-1376. 


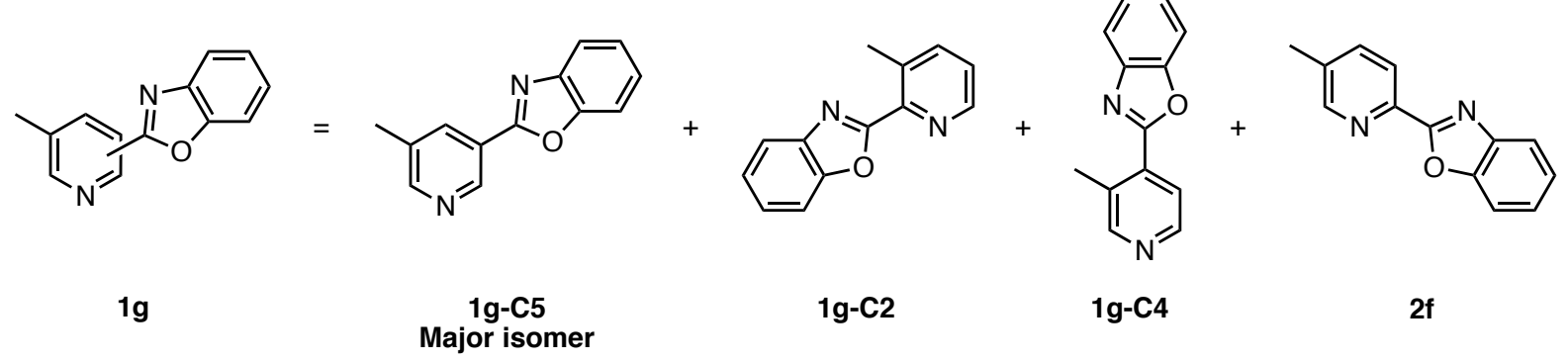

The product $\mathbf{1 g}$ (Figure 3, $32.7 \mathrm{mg}$, white solid) was obtained as a mixture of regioisomers (1g-C5, 1g-C2, 1g-C4, and 2f), which were inseparable with silica-gel column chromatography or gel permeation chromatography. The ratio of regioisomers was determined by ${ }^{1} \mathrm{H}$ NMR spectroscopy.

2-(3-Methylpyridin-5-yl)benzo[d]oxazole (1g-C5): The authentic product was synthesized with the reported procedure. ${ }^{\mathrm{S} 8}{ }^{1} \mathrm{H}$ NMR $\left(\mathrm{CDCl}_{3}\right) \delta 2.46(\mathrm{~s}, 1 \mathrm{H}), 7.38-7.40(\mathrm{~m}, 2 \mathrm{H}), 7.61-7.62$ (m, 1H), 7.79-7.80 (m, 1H), $8.34(\mathrm{~s}, 1 \mathrm{H}), 8.60(\mathrm{~s}, 1 \mathrm{H}), 9.28(\mathrm{~s}, 1 \mathrm{H}),{ }^{13} \mathrm{C} \mathrm{NMR}\left(\mathrm{CDCl}_{3}\right) \delta$ $18.55,110.93,120.41,123.24,125.07,125.82,133.67,135.26,142.00,146.21,150.96$, 152.91, 161.19; HR-MS (ESI-MS, positive): $m / z=211.0863$. calcd for $\mathrm{C}_{13} \mathrm{H}_{11} \mathrm{~N}_{2} \mathrm{O}: 211.0866$ $[M+\mathrm{H}]^{+}$.

2-(3-Methylpyridin-2-yl)benzo[d]oxazole (1g-C2): The authentic product was synthesized with the reported procedure. ${ }^{\mathrm{S} 8}{ }^{1} \mathrm{H}$ NMR $\left(\mathrm{CDCl}_{3}\right) \delta 2.88(\mathrm{~s}, 3 \mathrm{H}), 7.34-7.42(\mathrm{~m}, 3 \mathrm{H}), 7.68-7.71$ (m, 2H), $7.85(\mathrm{~d}, J=7.2 \mathrm{~Hz}, 1 \mathrm{H}), 8.69(\mathrm{~s}, 1 \mathrm{H}) ;{ }^{13} \mathrm{C} \mathrm{NMR}\left(\mathrm{CDCl}_{3}\right) \delta 21.26,111.33,120.84$, $124.77,125.06,126.07,135.57,140.04,142.12$, 144.54, 147.60, 150.48, 161.56; HR-MS (ESI-MS, positive): $m / z=211.0864$. calcd for $\mathrm{C}_{13} \mathrm{H}_{11} \mathrm{~N}_{2} \mathrm{O}: 211.0866[M+\mathrm{H}]^{+}$.

2-(3-Methylpyridin-4-yl)benzo[d]oxazole (1g-C4): The ${ }^{1} \mathrm{H}$ NMR data was assigned by analogy to compound 1a-C4. ${ }^{1} \mathrm{H}$ NMR $\left(\mathrm{CDCl}_{3}\right) \delta$ 7.34-7.44 (m, 1H), 7.61-7.72 (m, 2H), 7.85 $(\mathrm{d}, J=8.4 \mathrm{~Hz}, 1 \mathrm{H}), 7.04(\mathrm{~d}, J=4.8 \mathrm{~Hz}, 1 \mathrm{H}), 8.64(\mathrm{~d}, J=5.4 \mathrm{~Hz}, 1 \mathrm{H}), 8.66(\mathrm{~s}, 1 \mathrm{H})$.

2-(3-Methylpyridin-6-yl)benzo[d]oxazole (2f): The compound data was determined from the pure form of $\mathbf{2 f}$, which was synthesized through the C2-selective reaction. ${ }^{1} \mathrm{H}$ NMR $\left(\mathrm{CDCl}_{3}\right) \delta 2.44(\mathrm{~s}, 3 \mathrm{H}), 7.38-7.40(\mathrm{~m}, 2 \mathrm{H}), 7.66(\mathrm{dd}, J=5.4,2.4 \mathrm{~Hz}, 1 \mathrm{H}), 7.69(\mathrm{dd}, J=8.4$, $1.8 \mathrm{~Hz}, 1 \mathrm{H}), 7.81(\mathrm{dd}, J=7.2,1.8 \mathrm{~Hz}, 1 \mathrm{H}), 8.25(\mathrm{~d}, J=8.4 \mathrm{~Hz}, 1 \mathrm{H}), 8.64(\mathrm{~s}, 1 \mathrm{H}) ;{ }^{13} \mathrm{C} \mathrm{NMR}$ $\left(\mathrm{CDCl}_{3}\right) \delta 18.85,111.35,120.67,123.23,125.02,125.97,136,10,137.67,142.04,143.71$, 151.00, 151.19, 161.93; HR-MS (ESI-MS, positive): $m / z=211.0861$ calcd for $\mathrm{C}_{13} \mathrm{H}_{11} \mathrm{~N}_{2} \mathrm{O}$ : $211.0866[M+\mathrm{H}]^{+}$. 
HR-MS and ${ }^{13} \mathrm{C}$ NMR data for the mixture of 1g-C5, 1g-C2, 1g-C4, and $2 \mathrm{f}:{ }^{13} \mathrm{C}$ NMR $\left(\mathrm{CDCl}_{3}\right) \delta 18.56,18.86,19.23,21.30,110.95,111.01,111.36,120.42,120.68,120.87,120.96$, $122.54,123.24,124.81,125.03,125.08,125.83,125.98,126.10,126.32,132.62,132.20$, $133.67,135.28,135.61,136.11,137.68,140.09$, 142.01, 142.05, 142.14, 143.72, 146.22, $147.64,148.03,150.50,150.96,151.01,151.20,152.92,153.13,161.20,161.94$; HR-MS (ESI-MS, positive): $m / z=211.0861$. calcd for $\mathrm{C}_{13} \mathrm{H}_{11} \mathrm{~N}_{2} \mathrm{O}: 211.0866[M+\mathrm{H}]^{+}$. 


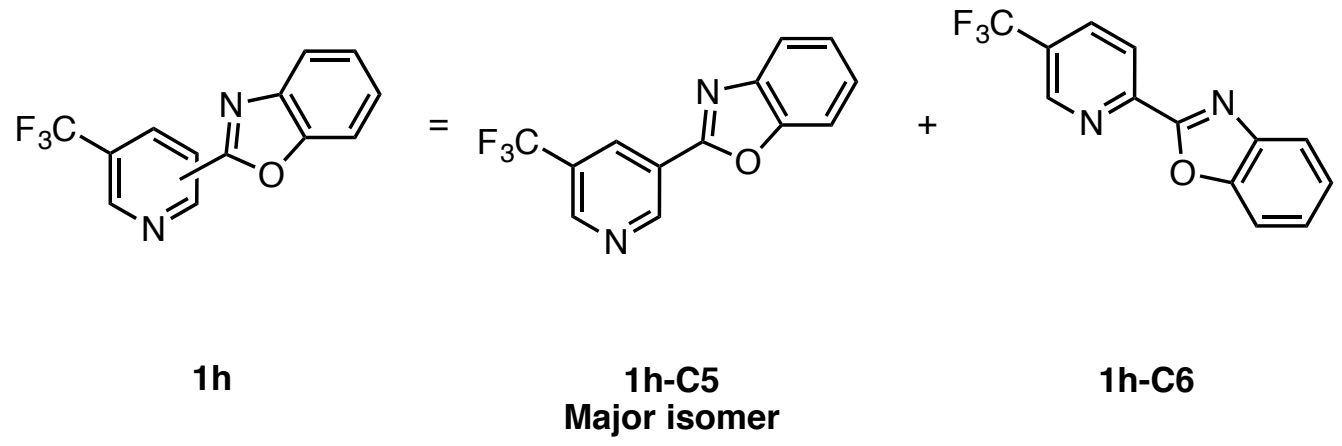

The product $\mathbf{1 h}$ (Figure 3, $20.1 \mathrm{mg}$, white solid) was obtained as a mixture of regioisomers (1h-C5 and 1h-C6), which were inseparable with silica-gel column chromatography or gel permeation chromatography. The ratio of regioisomers was determined by ${ }^{1} \mathrm{H}$ NMR spectroscopy.

2-(3-Trifluoromethylpyridin-5-yl)benzo[d]oxazole (1h-C5): ${ }^{1} \mathrm{H}$ NMR $\left(\mathrm{CDCl}_{3}\right) \delta$ 7.41-7.48 $(\mathrm{m}, 2 \mathrm{H}), 7.65(\mathrm{~d}, J=7.2 \mathrm{~Hz}, 1 \mathrm{H}), 7.83(\mathrm{~d}, J=6.6 \mathrm{~Hz}, 1 \mathrm{H}), 8.76(\mathrm{~s}, 1 \mathrm{H}), 9.03(\mathrm{~s}, 1 \mathrm{H}), 9.64$ (s, $1 \mathrm{H}) ;{ }^{13} \mathrm{C} \mathrm{NMR}\left(\mathrm{CDCl}_{3}\right) \delta 111.02,120.68,123.13\left(\mathrm{q}, J_{\mathrm{C}-\mathrm{F}}=272 \mathrm{~Hz}\right), 123.76,125.37,126.88$, $127.21\left(\mathrm{q}, J_{\mathrm{C}-\mathrm{F}}=33 \mathrm{~Hz}\right), 131.88\left(\mathrm{q}, J_{\mathrm{C}-\mathrm{F}}=3.0 \mathrm{~Hz}\right), 141.66,148.56\left(\mathrm{q}, J_{\mathrm{C}-\mathrm{F}}=4.4 \mathrm{~Hz}\right), 150.92$, $151.54,159.22$.

2-(3-Trifluoromethylpyridin-6-yl)benzo[d]oxazole (1h-C6): The authentic product was synthesized with the reported procedure. ${ }^{\mathrm{S} 8}{ }^{1} \mathrm{H} \mathrm{NMR}\left(\mathrm{CDCl}_{3}\right) \delta 7.42-7.48(\mathrm{~m}, 2 \mathrm{H}), 7.70(\mathrm{~d}, J=$ $7.8 \mathrm{~Hz}, 1 \mathrm{H}), 7.87(\mathrm{~d}, J=7.8 \mathrm{~Hz}, 1 \mathrm{H}), 8.15(\mathrm{dd}, J=8.4,2.4 \mathrm{~Hz}, 1 \mathrm{H}), 8.50$ (d, $J=8.4 \mathrm{~Hz}, 1 \mathrm{H})$, $9.07(\mathrm{~s}, 1 \mathrm{H}) ;{ }^{13} \mathrm{C} \mathrm{NMR}\left(\mathrm{CDCl}_{3}\right) \delta 111.61,121.24,123.23,123.31\left(\mathrm{q}, J_{\mathrm{C}-\mathrm{F}}=272 \mathrm{~Hz}\right), 125.52$, $127.00,128.17\left(\mathrm{q}, J_{\mathrm{C}-\mathrm{F}}=34 \mathrm{~Hz}\right), 134.65\left(\mathrm{q}, J_{\mathrm{C}-\mathrm{F}}=2.9 \mathrm{~Hz}\right), 141.84,147.39\left(\mathrm{q}, J_{\mathrm{C}-\mathrm{F}}=4.4 \mathrm{~Hz}\right)$, 149.25, 151.40, 160.32; HR-MS (ESI-MS, positive): $\mathrm{m} / z=265.0583$. calcd for $\mathrm{C}_{13} \mathrm{H}_{8} \mathrm{~F}_{3} \mathrm{~N}_{2} \mathrm{O}$ : $265.0583[M+\mathrm{H}]^{+}$.

HR-MS data for the mixture of 1h-C5 and 1h-C6: HR-MS (ESI-MS, positive): $\mathrm{m} / \mathrm{z}=$ 265.0578. calcd for $\mathrm{C}_{13} \mathrm{H}_{8} \mathrm{~F}_{3} \mathrm{~N}_{2} \mathrm{O}: 265.0583[M+\mathrm{H}]^{+}$. 


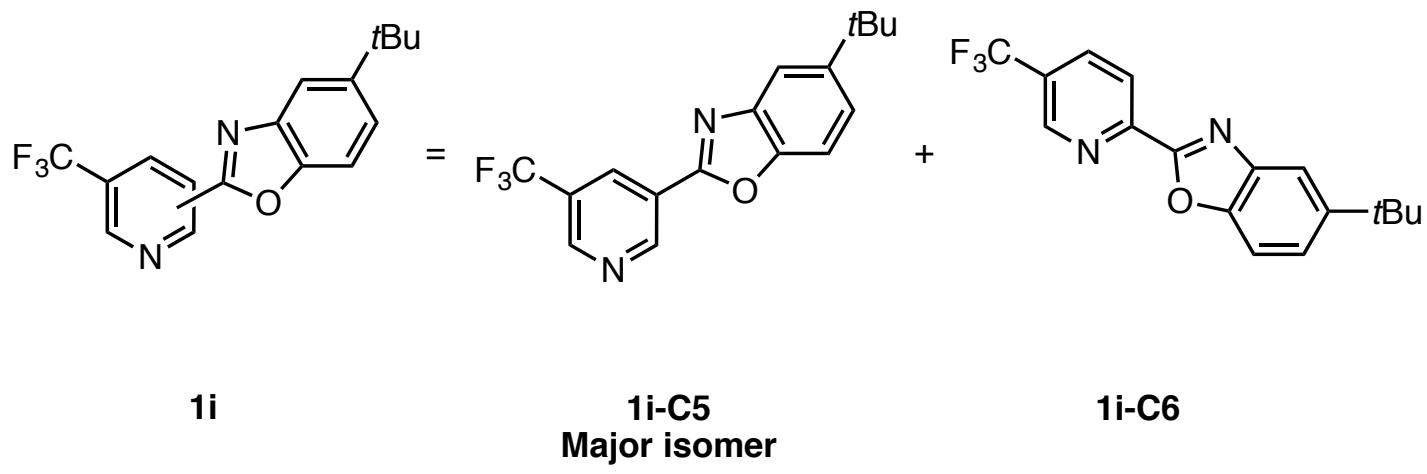

The product 1i (Figure 3, $26.4 \mathrm{mg}$, white solid) was obtained as a mixture of regioisomers (1iC5 and 1i-C6), which were inseparable with silica-gel column chromatography or gel permeation chromatography. The ratio of regioisomers was determined by ${ }^{1} \mathrm{H}$ NMR spectroscopy.

5-(tert-Butyl)-2-(3-trifluoromethylpyridin-5-yl)benzo[d]oxazole (1i-C5): The ${ }^{1} \mathrm{H}$ NMR data was assigned by analogy to compound $\mathbf{1 h} .{ }^{1} \mathrm{H}$ NMR $\left(\mathrm{CDCl}_{3}\right) \delta 1.41(\mathrm{~s}, 9 \mathrm{H}), 7.50-7.56$ $(\mathrm{m}, 1 \mathrm{H}), 7.60(\mathrm{~d}, J=6.6 \mathrm{~Hz}, 1 \mathrm{H}), 7.84-7.90(\mathrm{~m}, 1 \mathrm{H}), 8.13(\mathrm{~d}, J=8.4 \mathrm{~Hz}, 1 \mathrm{H}), 8.48(\mathrm{~d}, J=$ $8.4 \mathrm{~Hz}, 1 \mathrm{H}), 9.05(\mathrm{~s}, 1 \mathrm{H})$.

5-(tert-Butyl)-2-(3-trifluoromethylpyridin-6-yl)benzo[d]oxazole (1i-C6): The ${ }^{1} \mathrm{H}$ NMR data was assigned by analogy to compound 1h-C6. ${ }^{1} \mathrm{H}$ NMR $\left(\mathrm{CDCl}_{3}\right) \delta 1.41(\mathrm{~s}, 9 \mathrm{H}), 7.50$ $7.56(\mathrm{~m}, 2 \mathrm{H}), 7.84-7.90(\mathrm{~m}, 1 \mathrm{H}), 8.74(\mathrm{~s}, 1 \mathrm{H}), 9.01(\mathrm{~s}, 1 \mathrm{H}), 9.63(\mathrm{~s}, 1 \mathrm{H})$.

HR-MS and ${ }^{13} \mathrm{C}$ NMR data for the mixture of 1i-C5 and 1i-C6: ${ }^{13} \mathrm{C} \mathrm{NMR}\left(\mathrm{CDCl}_{3}\right) \delta 31.89$, $35.22,110.26,110.74,117.18,117.48,123.08,123.28\left(\mathrm{q}, J_{\mathrm{C}-\mathrm{F}}=271 \mathrm{~Hz}\right), 123.34\left(\mathrm{q}, J_{\mathrm{C}-\mathrm{F}}=270\right.$ $\mathrm{Hz}), 124.04,124.41,124.97,127.30\left(\mathrm{q}, J_{\mathrm{C}-\mathrm{F}}=35 \mathrm{~Hz}\right), 127.98\left(\mathrm{q}, J_{\mathrm{C}-\mathrm{F}}=33 \mathrm{~Hz}\right), 131.83\left(\mathrm{q}, J_{\mathrm{C}-\mathrm{F}}\right.$ $=4.4 \mathrm{~Hz}), 134.57,141.74,141.78,147.32\left(\mathrm{q}, J_{\mathrm{C}-\mathrm{F}}=4.4 \mathrm{~Hz}\right), 148.50\left(\mathrm{q}, J_{\mathrm{C}-\mathrm{F}}=2.9 \mathrm{~Hz}\right), 149.08$, 149.19, 149.40, 149.44, 151.57, 159.41, 160.43; HR-MS (ESI-MS, positive): $\mathrm{m} / z=321.1202$. calcd for $\mathrm{C}_{17} \mathrm{H}_{16} \mathrm{~F}_{3} \mathrm{~N}_{2} \mathrm{O}: 321.1209[M+\mathrm{H}]^{+}$. 
<smiles>Cc1ccc(-c2nc3cc(C(C)(C)C)ccc3o2)c(C)n1</smiles>

$1 \mathrm{j}$

5-(tert-Butyl)-2-(2,6-dimethylpyridin-3-yl)benzo[d] oxazole: (Figure 3, $20.2 \mathrm{mg}$, colorless liquid) ${ }^{1} \mathrm{H} \mathrm{NMR}\left(\mathrm{CDCl}_{3}\right) \delta 1.41(\mathrm{~s}, 9 \mathrm{H}), 2.61(\mathrm{~s}, 1 \mathrm{H}), 3.03(\mathrm{~s}, 1 \mathrm{H}), 7.15(\mathrm{~d}, J=8.4 \mathrm{~Hz}, 1 \mathrm{H})$, $7.43(\mathrm{dd}, J=8.4,1.2 \mathrm{~Hz}, 1 \mathrm{H}), 7.50(\mathrm{~d}, J=8.4 \mathrm{~Hz}, 1 \mathrm{H}), 7.83(\mathrm{~d}, J=1.2 \mathrm{~Hz}, 1 \mathrm{H}), 8.32(\mathrm{~d}, J=$ $7.8 \mathrm{~Hz}, 1 \mathrm{H}) ;{ }^{13} \mathrm{C} \mathrm{NMR}\left(\mathrm{CDCl}_{3}\right) \delta 24.86,25.38,31.97,35.16,109.81,116.89,119.83,120.91$, 123.23, 137.69, 142.14, 148.37, 148.59, 157.87, 160.17, 162.34; HR-MS (ESI-MS, positive): $m / z=281.1642$. calcd for $\mathrm{C}_{18} \mathrm{H}_{21} \mathrm{~N}_{2} \mathrm{O}: 281.1648[M+\mathrm{H}]^{+}$. 


\section{Characterization Data for C2-Selective Reaction}<smiles>c1ccc(-c2nc3ccccc3o2)nc1</smiles>

$2 a$

2-(Pyridin-2-yl)benzo[d] oxazole (2a): (Figure 4, $36.3 \mathrm{mg}$, white solid) The compound data for $\mathbf{2 a}$ was presented in the $\mathrm{C} 3$-selective reaction section (vide supra).

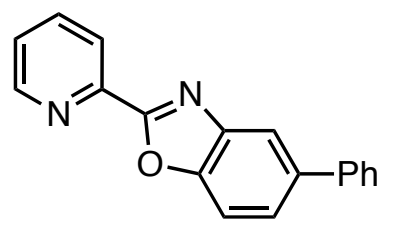

$2 b$

5-Phenyl-2-(pyridin-2-yl)benzo[d] oxazole (2b): (Figure 4, $29.3 \mathrm{mg}$, light yellow solid) The compound data for $\mathbf{2 b}$ was presented in the $\mathrm{C} 3$-selective reaction section (vide supra).<smiles>CC(C)(C)c1ccc2oc(-c3ccccn3)nc2c1</smiles>

2c

5-(tert-Butyl)-2-(pyridin-2-yl)benzo[d]oxazole (2c): (Figure 4, $30.2 \mathrm{mg}$, white solid) The compound data for $\mathbf{2 c}$ was presented in the $\mathrm{C} 3$-selective reaction section (vide supra).

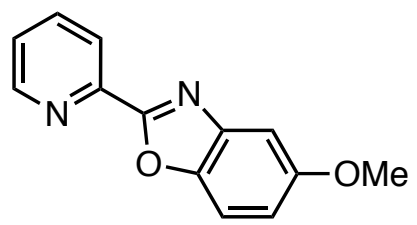

2d

5-Methoxy-2-(pyridin-2-yl)benzo[d] oxazole (2d): (Figure 4, $35.7 \mathrm{mg}$, white solid) ${ }^{1} \mathrm{H}$ NMR $\left(\mathrm{CDCl}_{3}\right) \delta 3.89(\mathrm{~s}, 3 \mathrm{H}), 7.02(\mathrm{~d}, J=9.0 \mathrm{~Hz}, 1 \mathrm{H}), 7.29(\mathrm{~s}, 1 \mathrm{H}), 7.45(\mathrm{t}, J=6.6 \mathrm{~Hz}, 1 \mathrm{H}), 7.55$ $(\mathrm{dd}, J=9.0,1.8 \mathrm{~Hz}, 1 \mathrm{H}), 7.89(\mathrm{t}, J=7.8 \mathrm{~Hz}, 1 \mathrm{H}), 8.34(\mathrm{~d}, J=8.4 \mathrm{~Hz}, 1 \mathrm{H}), 8.82(\mathrm{~d}, J=4.8 \mathrm{~Hz}$, $1 \mathrm{H}) ;{ }^{13} \mathrm{C}$ NMR $\left(\mathrm{CDCl}_{3}\right) \delta 56.16,103.33,111.60,115.22,123.50,125.63,137.25,142.85$, 
145.99, 146.37, 150.485, 157.88, 162.38; HR-MS (ESI-MS, positive): $\mathrm{m} / \mathrm{z}=227.0809$. calcd for $\mathrm{C}_{13} \mathrm{H}_{11} \mathrm{~N}_{2} \mathrm{O}_{2}: 227.0815[M+\mathrm{H}]^{+}$.<smiles>FC(F)(F)c1ccnc(-c2nc3ccccc3o2)c1</smiles>

$2 e$

2-(4-Trifluoromethylpyridin-2-yl)benzo[d] oxazole (2e): (Figure 4, $14.8 \mathrm{mg}$, white solid) ${ }^{1} \mathrm{H}$ NMR $\left(\mathrm{CDCl}_{3}\right) \delta 7.42-7.48(\mathrm{~m}, 2 \mathrm{H}), 7.68-7.71(\mathrm{~m}, 2 \mathrm{H}), 7.87(\mathrm{~d}, J=7.2 \mathrm{~Hz}, 1 \mathrm{H}), 8.61(\mathrm{~s}, 1 \mathrm{H})$, $9.01(\mathrm{~d}, J=4.8 \mathrm{~Hz}, 1 \mathrm{H}) ;{ }^{13} \mathrm{C} \mathrm{NMR}\left(\mathrm{CDCl}_{3}\right) \delta 111.56,119.43\left(\mathrm{q}, J_{\mathrm{C}-\mathrm{F}}=2.9 \mathrm{~Hz}\right), 121.11\left(\mathrm{q}, J_{\mathrm{C}-\mathrm{F}}\right.$ $=4.4 \mathrm{~Hz}), 121.16,122.61\left(\mathrm{q}, J_{\mathrm{C}-\mathrm{F}}=271 \mathrm{~Hz}\right), 125.50,126.87,139.93\left(\mathrm{q}, J_{\mathrm{C}-\mathrm{F}}=35 \mathrm{~Hz}\right), 141.79$, 147.59, 151.34, 151.49, 160.41; HR-MS (ESI-MS, positive): $\mathrm{m} / \mathrm{z}=265.0577$. calcd for $\mathrm{C}_{13} \mathrm{H}_{8} \mathrm{~F}_{3} \mathrm{~N}_{2} \mathrm{O}: 265.0583[M+\mathrm{H}]^{+}$.<smiles>Cc1ccc(-c2nc3ccccc3o2)nc1</smiles>

$2 f$

2-(5-Methylpyridin-2-yl)benzo[d]oxazole (2f): (Figure 4, $19.9 \mathrm{mg}$, white solid) The compound data for $\mathbf{2} \mathbf{f}$ was presented in the $\mathrm{C} 3$-selective reaction section (vide supra).

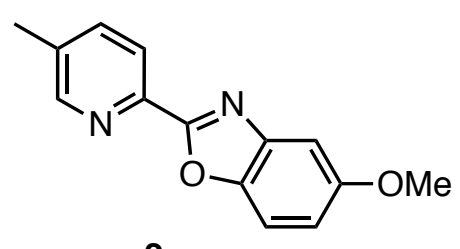

$2 g$

5-Methoxy-2-(5-methylpyridin-2-yl)benzo[d]oxazole (2g): (Figure 4, $23.3 \mathrm{mg}$, pale glay solid) ${ }^{1} \mathrm{H}$ NMR $\left(\mathrm{CDCl}_{3}\right) \delta 2.45(\mathrm{~s}, 1 \mathrm{H}), 3.88(\mathrm{~s}, 1 \mathrm{H}), 7.00(\mathrm{dd}, J=9.0,2.4 \mathrm{~Hz}, 1 \mathrm{H}), 7.28(\mathrm{~d}, J$ $=1.8 \mathrm{~Hz}, 1 \mathrm{H}), 7.53(\mathrm{~d}, J=9.0 \mathrm{~Hz}, 1 \mathrm{H}), 7.69(\mathrm{~d}, J=7.8 \mathrm{~Hz}, 1 \mathrm{H}), 8.23(\mathrm{~d}, J=8.4 \mathrm{~Hz}, 1 \mathrm{H})$, $8.63(\mathrm{~s}, 1 \mathrm{H}) ;{ }^{13} \mathrm{C} \mathrm{NMR}\left(\mathrm{CDCl}_{3}\right) \delta 18.84,56.16,103.27,111.50,114.85,123.07,135.96$, 
137.66, 142.89, 143.77, 145.90, 150.97, 157.80, 162.65; HR-MS (ESI-MS, positive): $\mathrm{m} / z=$ 241.0966. calcd for $\mathrm{C}_{14} \mathrm{H}_{13} \mathrm{~N}_{2} \mathrm{O}_{2}: 241.0972[M+\mathrm{H}]^{+}$. 


\section{6. ${ }^{1} \mathrm{H}$ and ${ }^{13} \mathrm{C}$ NMR Spectra}

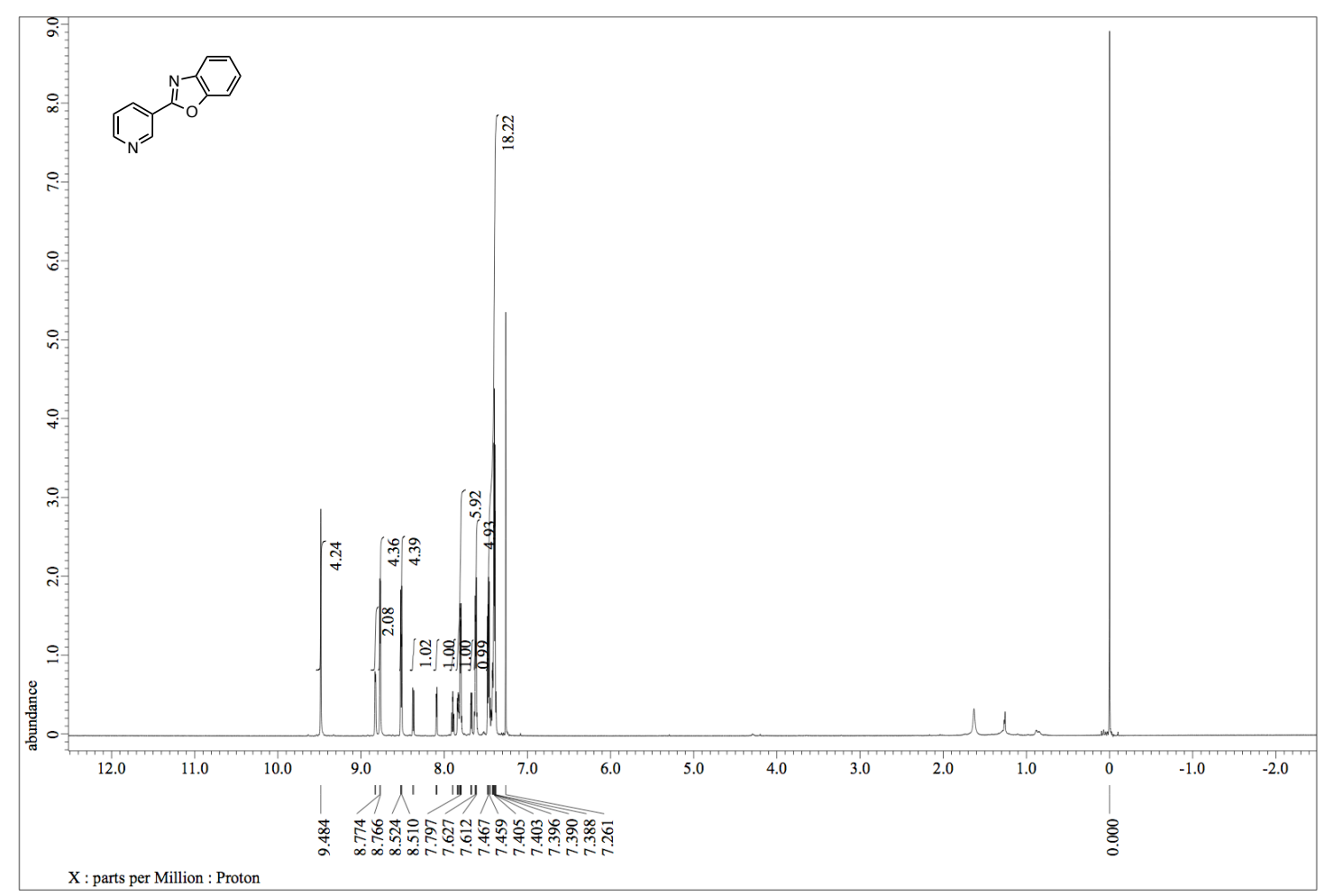

Figure S 1. ${ }^{1} \mathrm{H}$ NMR spectrum of $1 \mathbf{a}(\mathrm{C} 2 / \mathrm{C} 3 / \mathrm{C} 4=17: 74: 9)$.

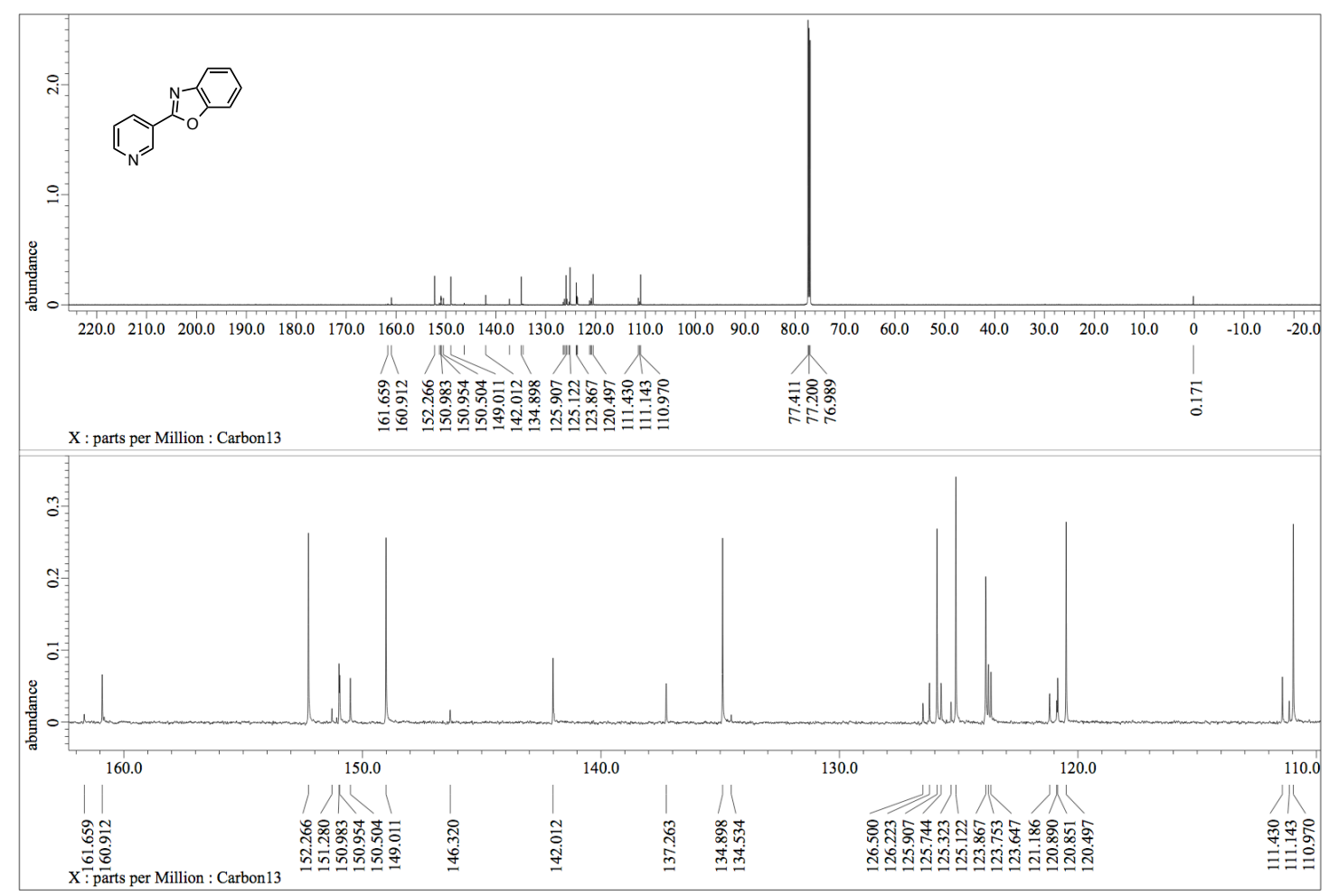

Figure S 2. ${ }^{13} \mathrm{C}$ NMR spectrum of $1 \mathbf{a}(\mathrm{C} 2 / \mathrm{C} 3 / \mathrm{C} 4=17: 74: 9)$. 


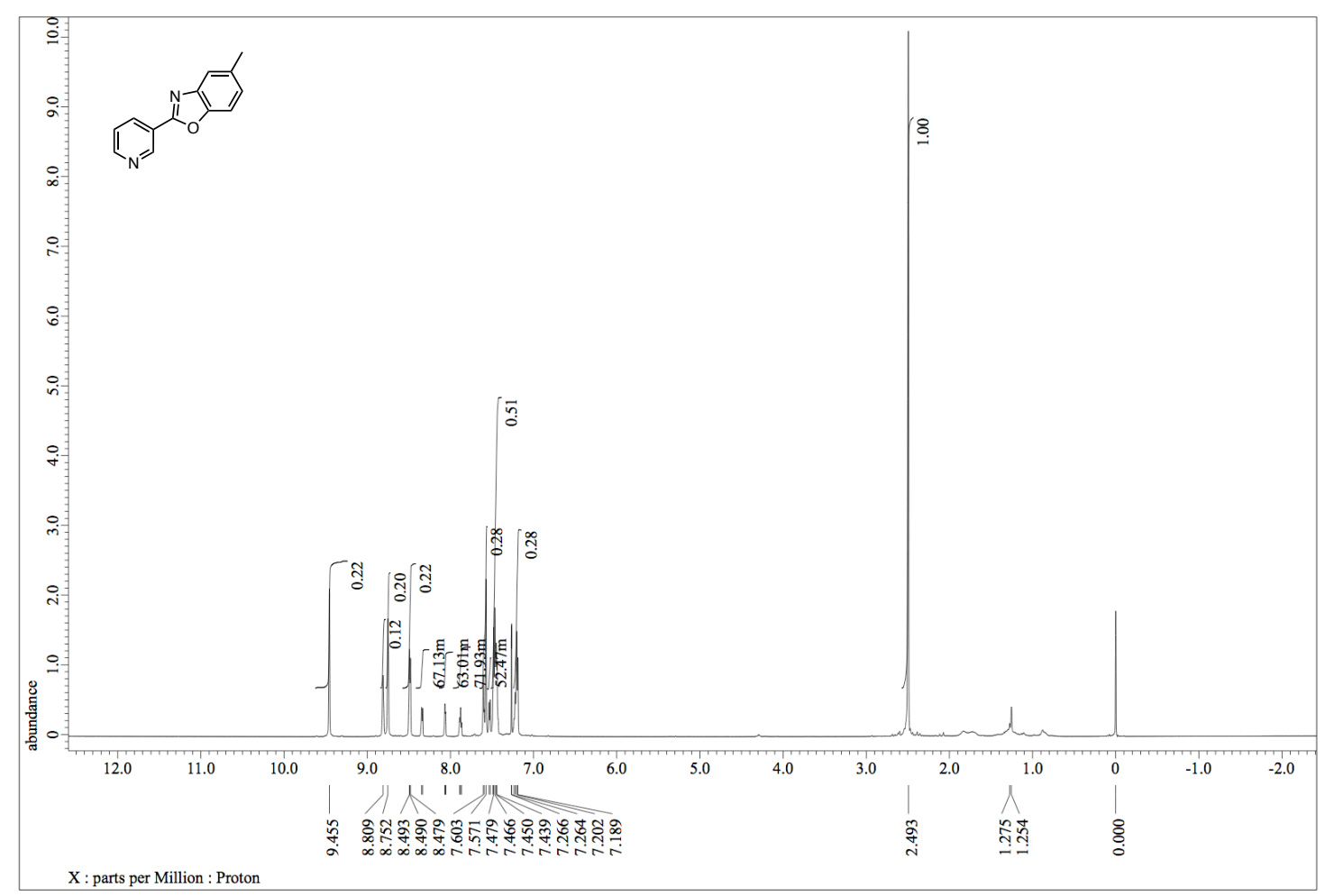

Figure S 3. ${ }^{1} \mathrm{H}$ NMR spectrum of $\mathbf{1 b}(\mathrm{C} 2 / \mathrm{C} 3 / \mathrm{C} 4=20: 65: 8)$.

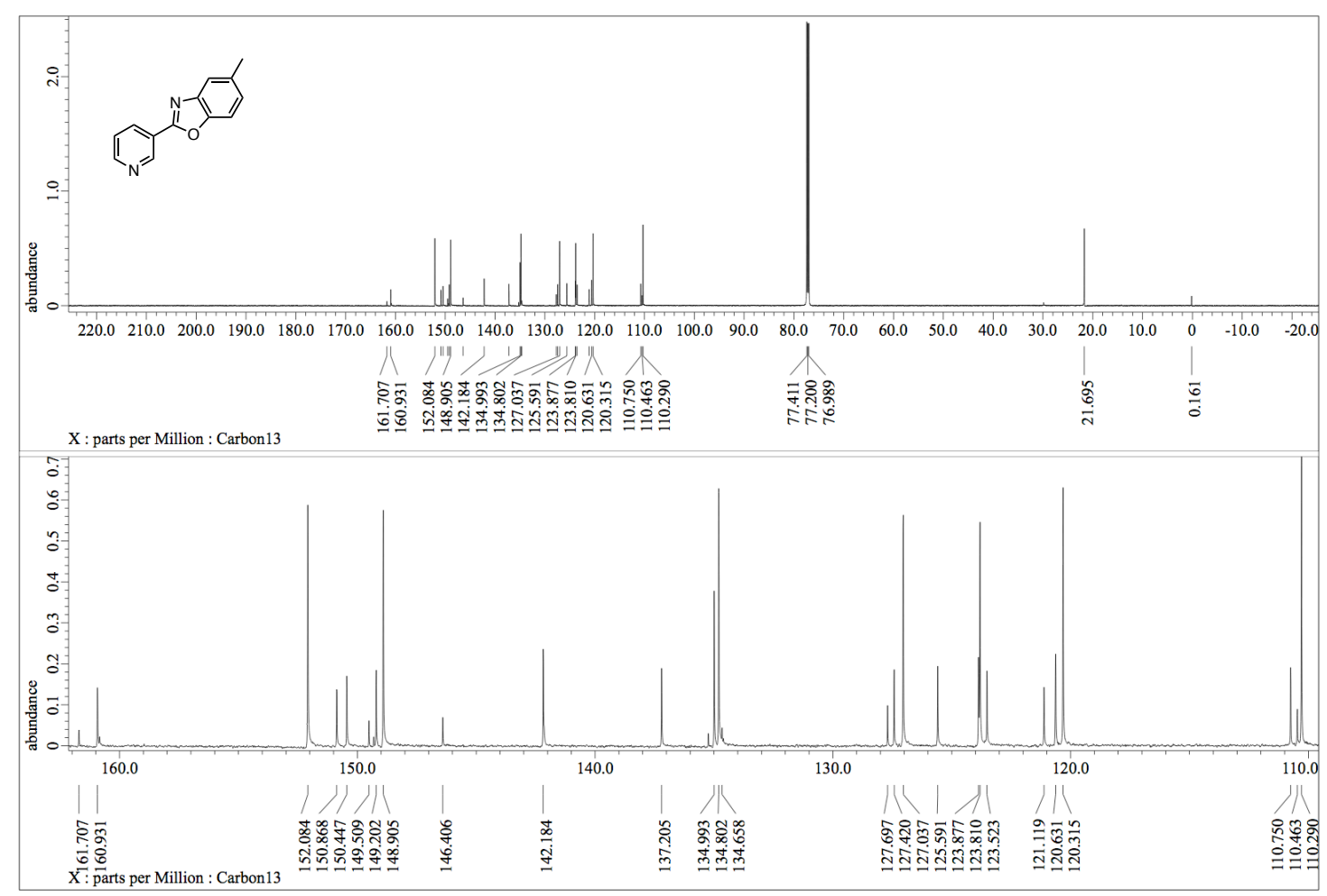

Figure S 4. ${ }^{13} \mathrm{C}$ NMR spectrum of $\mathbf{1 b}(\mathrm{C} 2 / \mathrm{C} 3 / \mathrm{C} 4=20: 65: 8)$. 


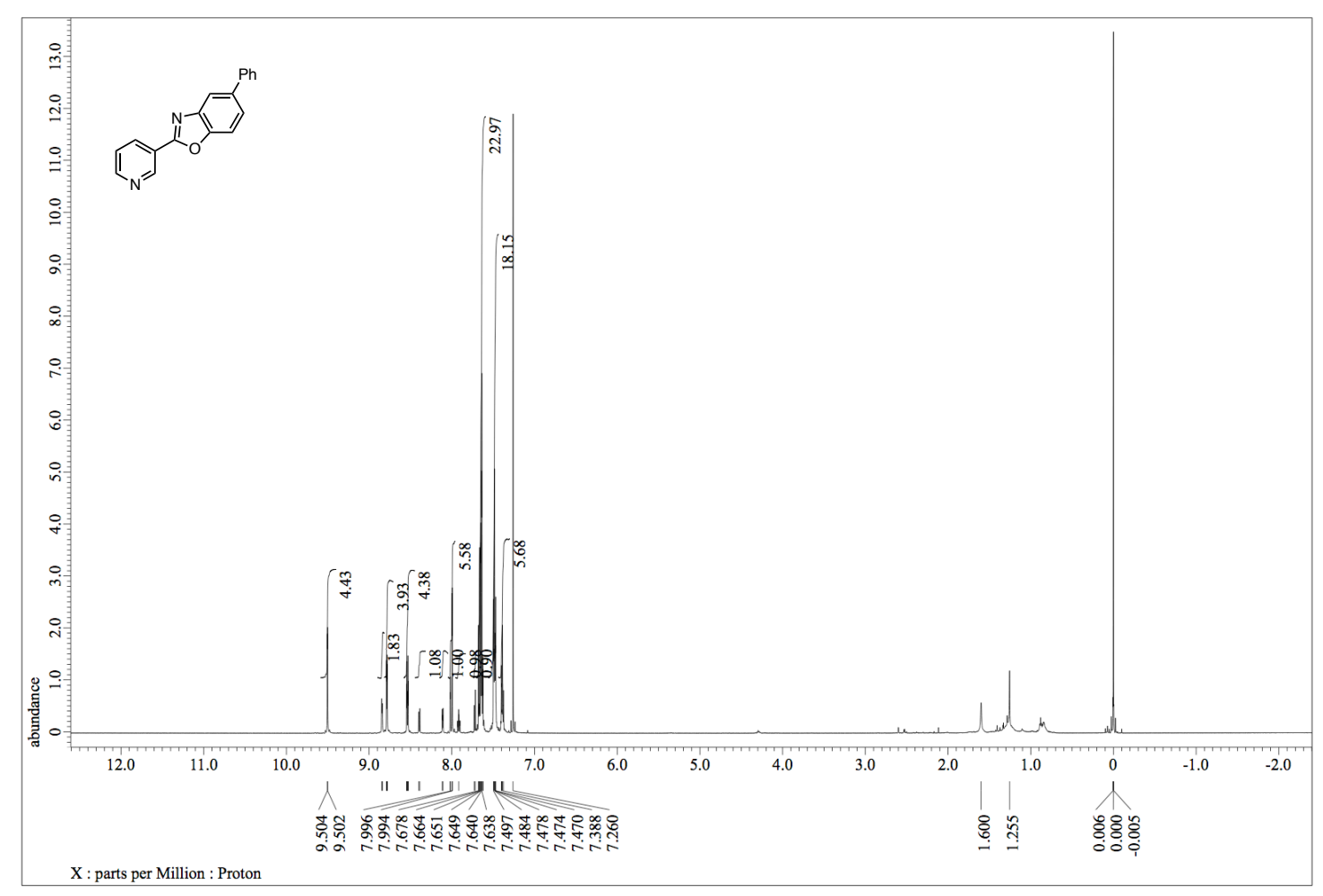

Figure S 5. ${ }^{1} \mathrm{H}$ NMR spectrum of $1 \mathbf{c}(\mathrm{C} 2 / \mathrm{C} 3 / \mathrm{C} 4=18: 73: 9)$.

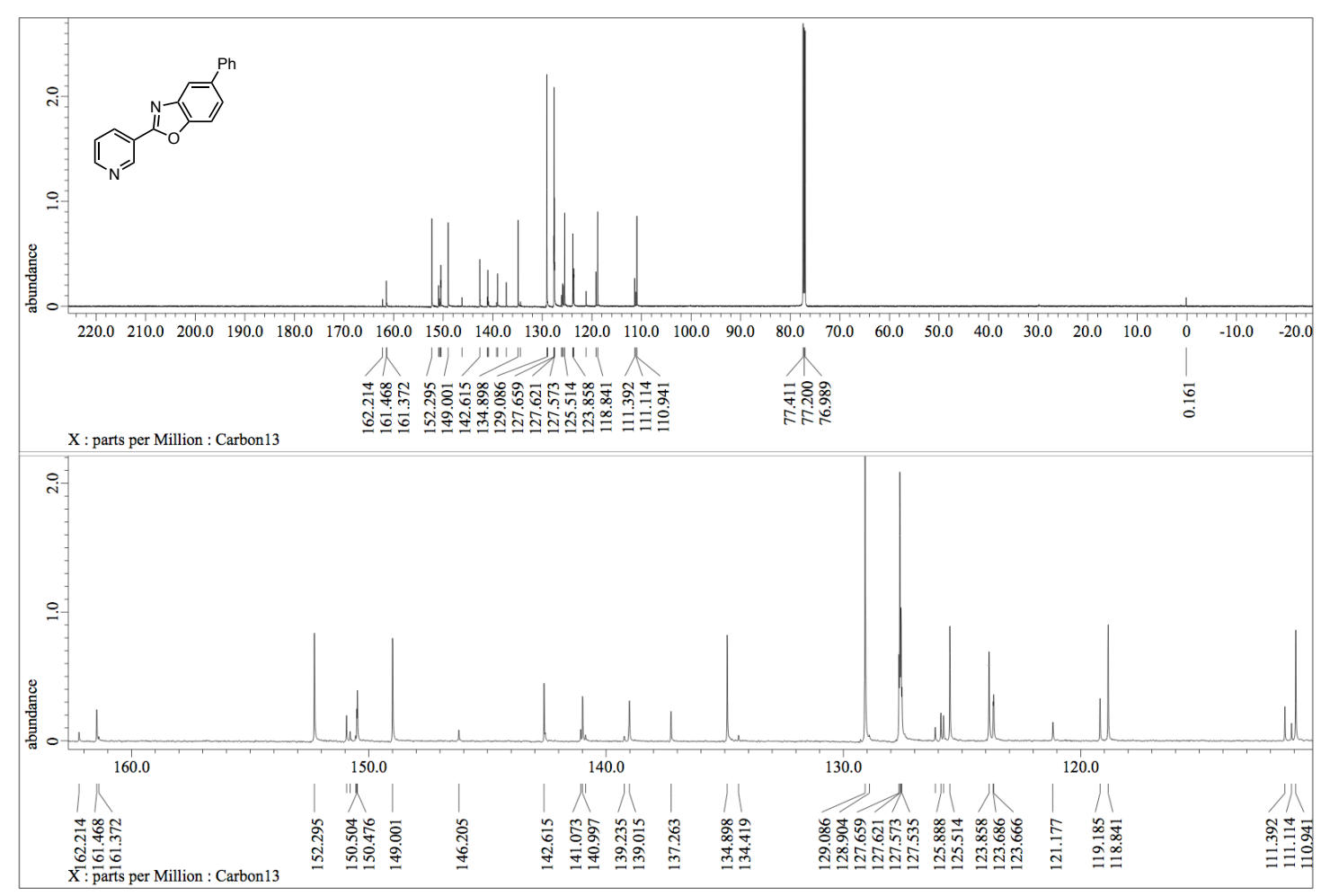

Figure S 6. ${ }^{13} \mathrm{C}$ NMR spectrum of $1 c(C 2 / C 3 / C 4=18: 73: 9)$. 


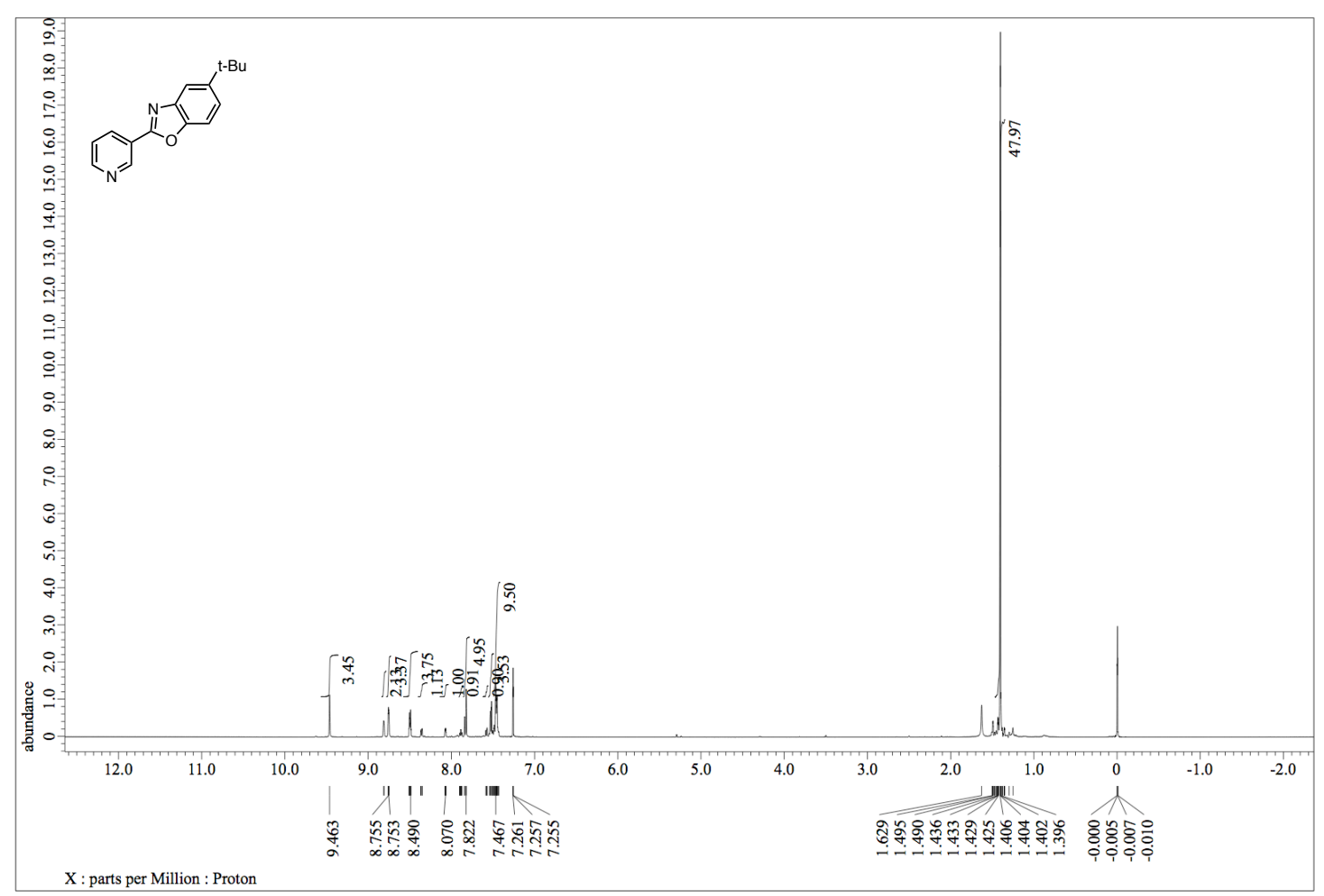

Figure S 7. ${ }^{1} \mathrm{H}$ NMR spectrum of $1 \mathbf{d}(\mathrm{C} 2 / \mathrm{C} 3 / \mathrm{C} 4=21: 69: 10)$.

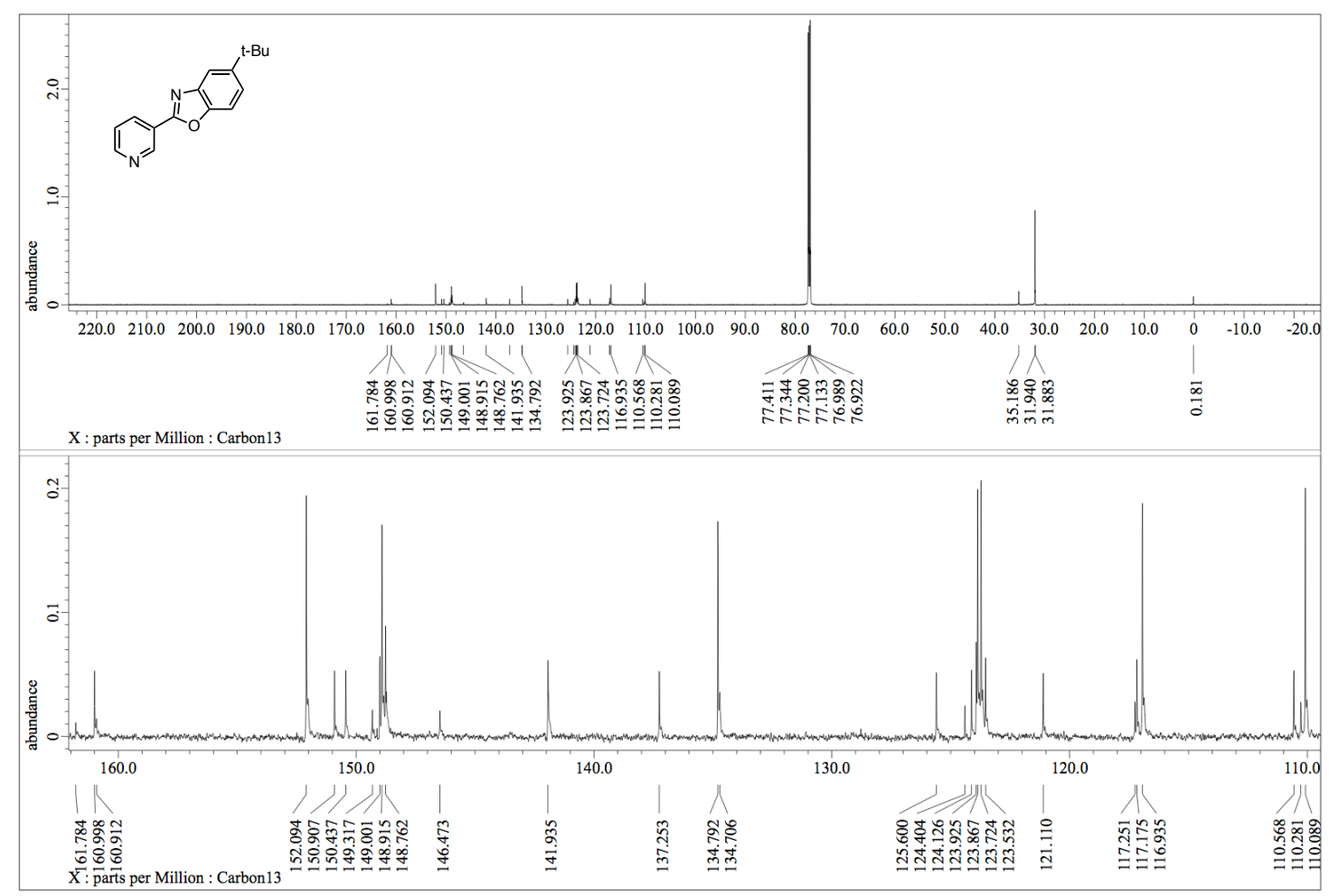

Figure S 8. ${ }^{13} \mathrm{C}$ NMR spectrum of $1 d(C 2 / C 3 / C 4=21: 69: 10)$. 


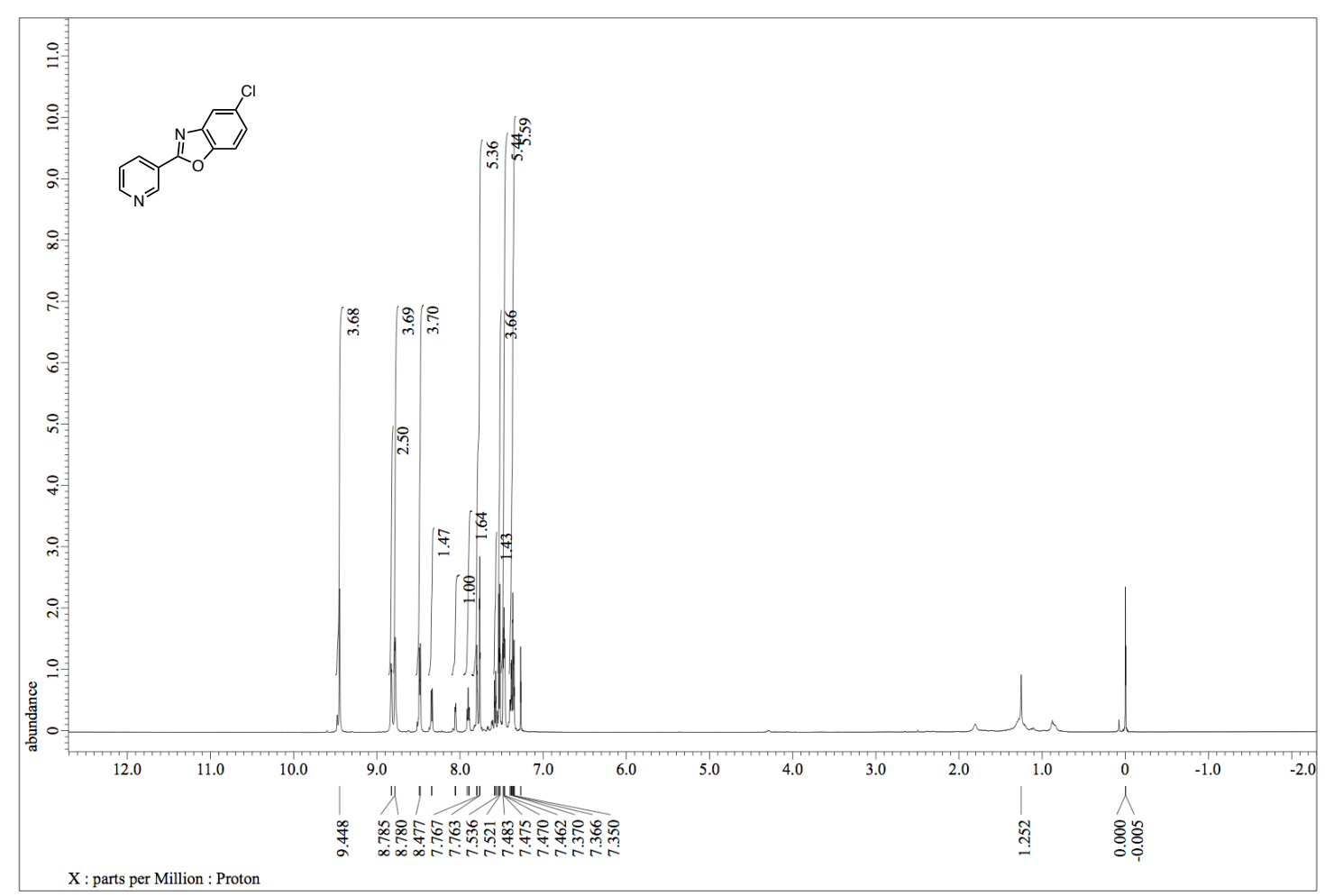

Figure S 9. ${ }^{1} \mathrm{H}$ NMR spectrum of 1 e $(\mathrm{C} 2 / \mathrm{C} 3 / \mathrm{C} 4=27: 65: 8)$.

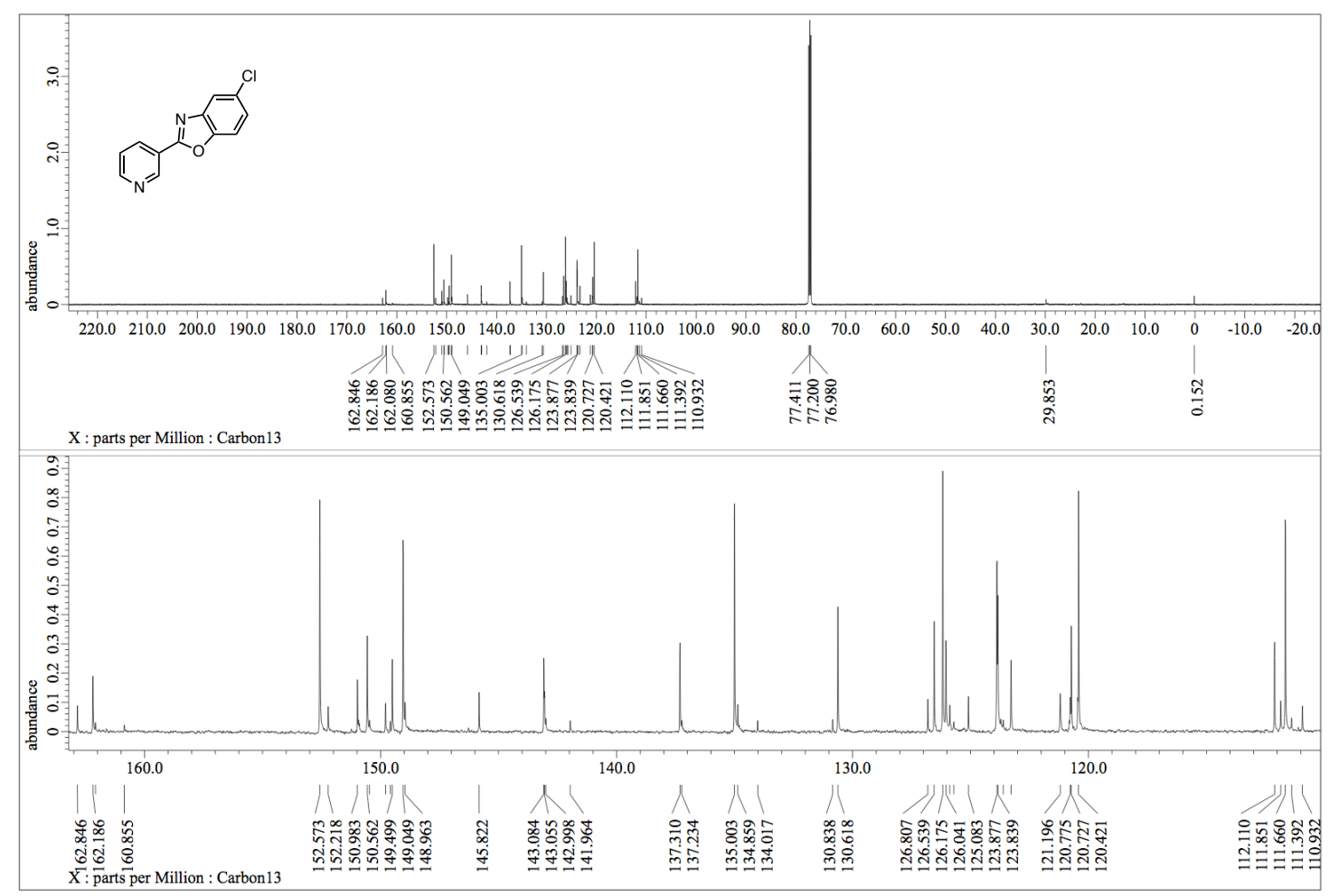

Figure S 10. ${ }^{13} \mathrm{C}$ NMR spectrum of $1 e(C 2 / C 3 / C 4=27: 65: 8)$. 


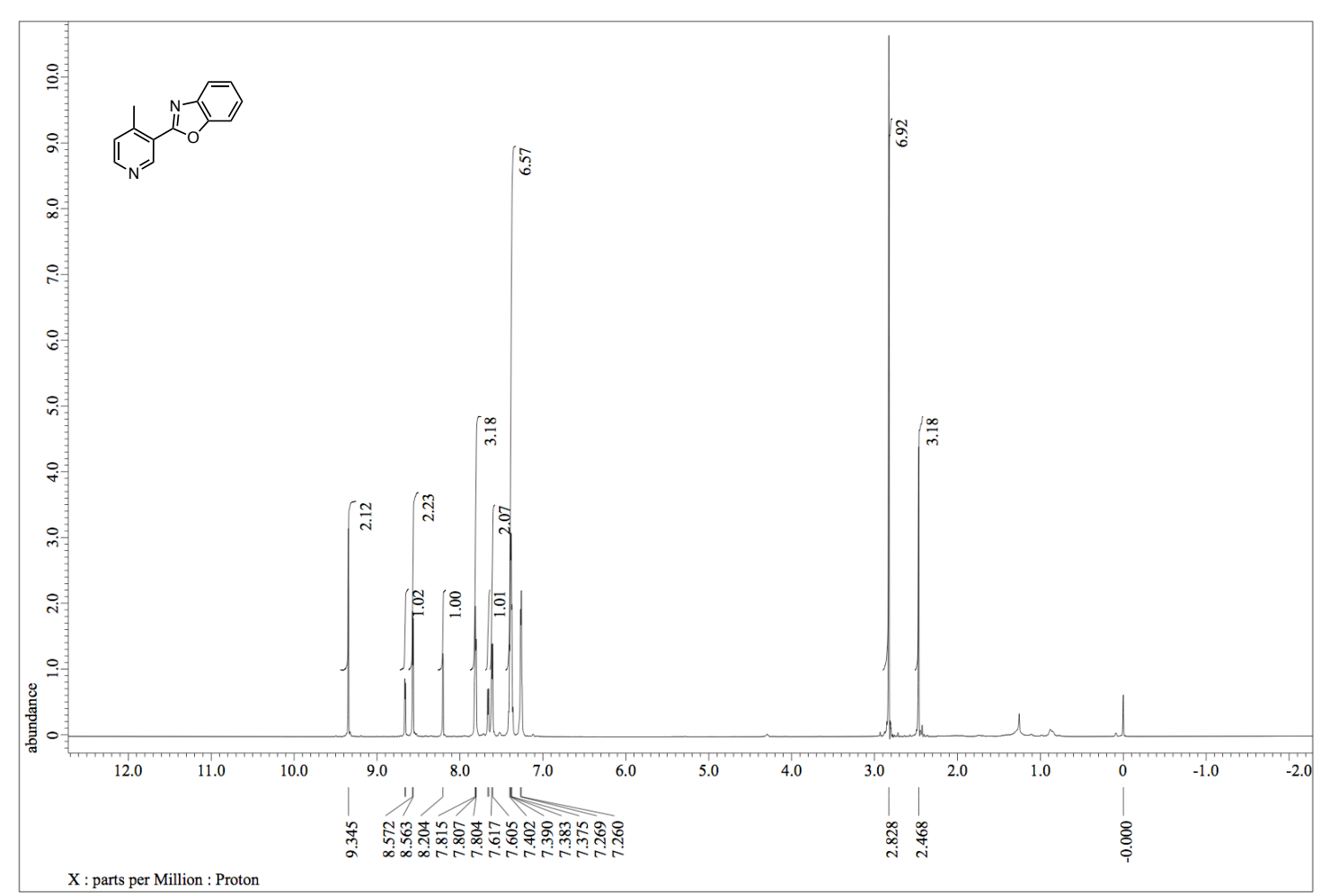

Figure S 11. ${ }^{1} \mathrm{H}$ NMR spectrum of $\mathbf{1 f}(\mathrm{C} 2 / \mathrm{C} 3=33: 67)$.

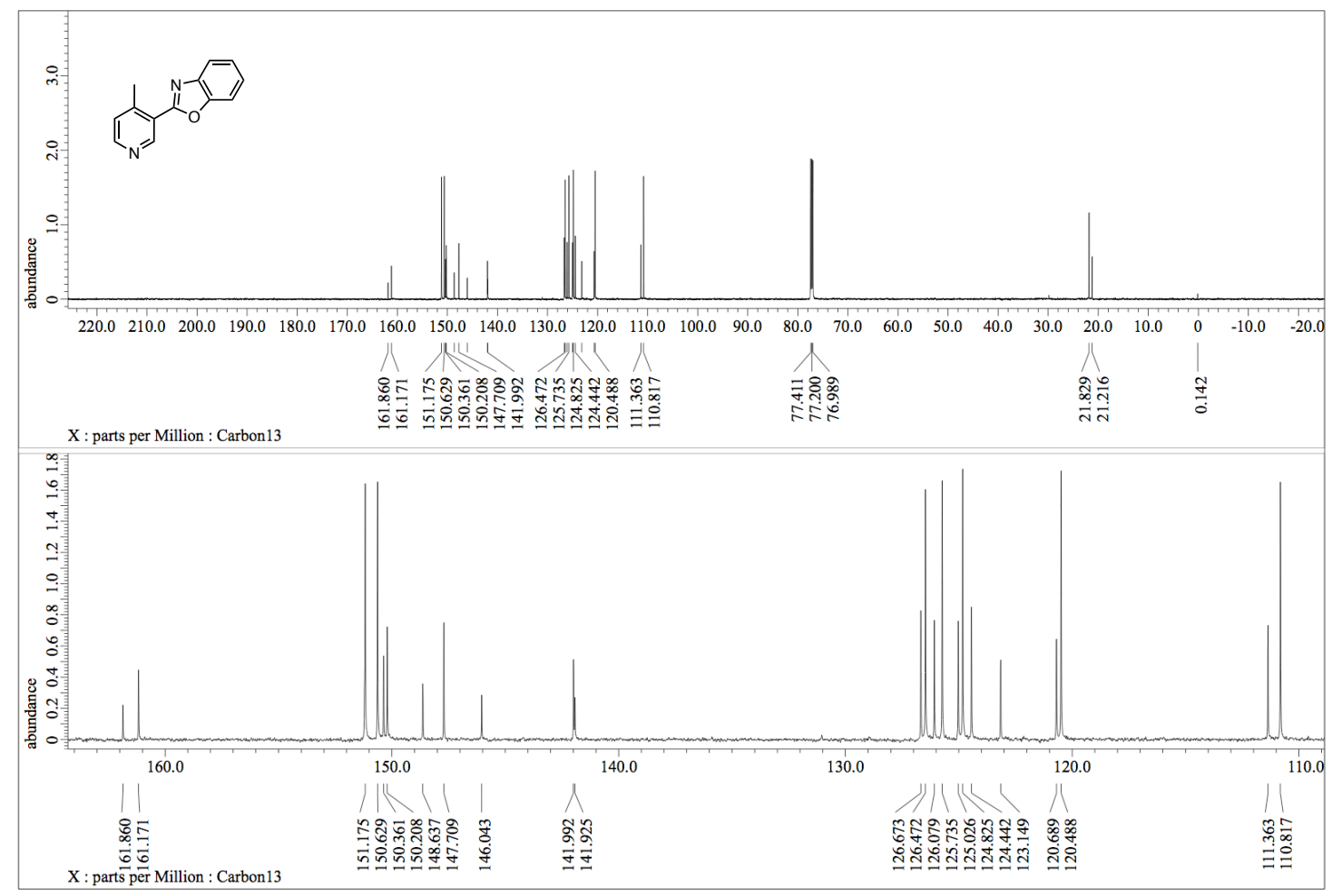

Figure S 12. ${ }^{13} \mathrm{C}$ NMR spectrum of $\mathbf{1 f}(\mathrm{C} 2 / \mathrm{C} 3=33: 67)$. 


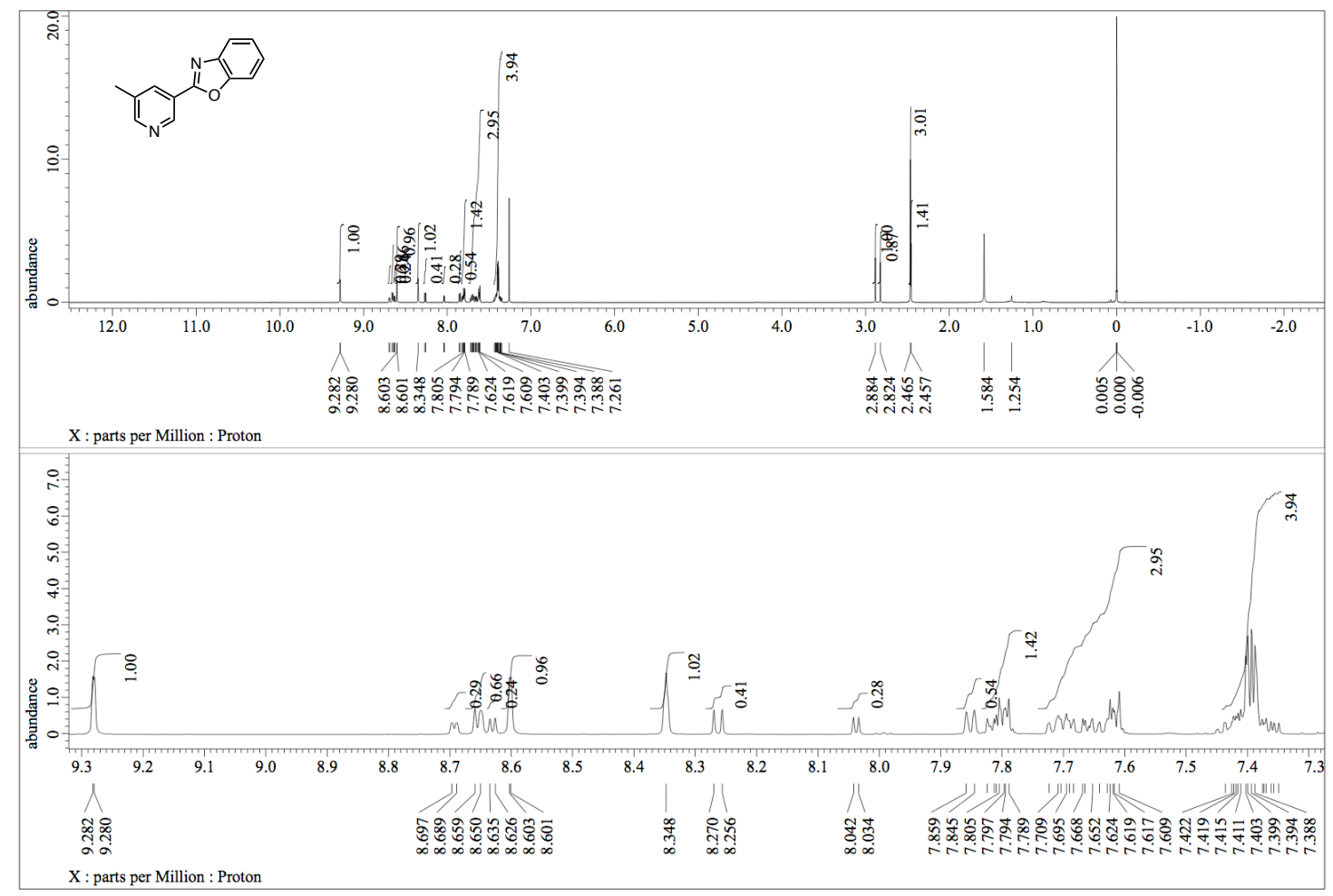

Figure S 13. ${ }^{1} \mathrm{H}$ NMR spectrum of $1 \mathrm{~g}(\mathrm{C} 2 / \mathrm{C} 4 / \mathrm{C} 5 / \mathrm{C} 6=14: 14: 51: 21)$.

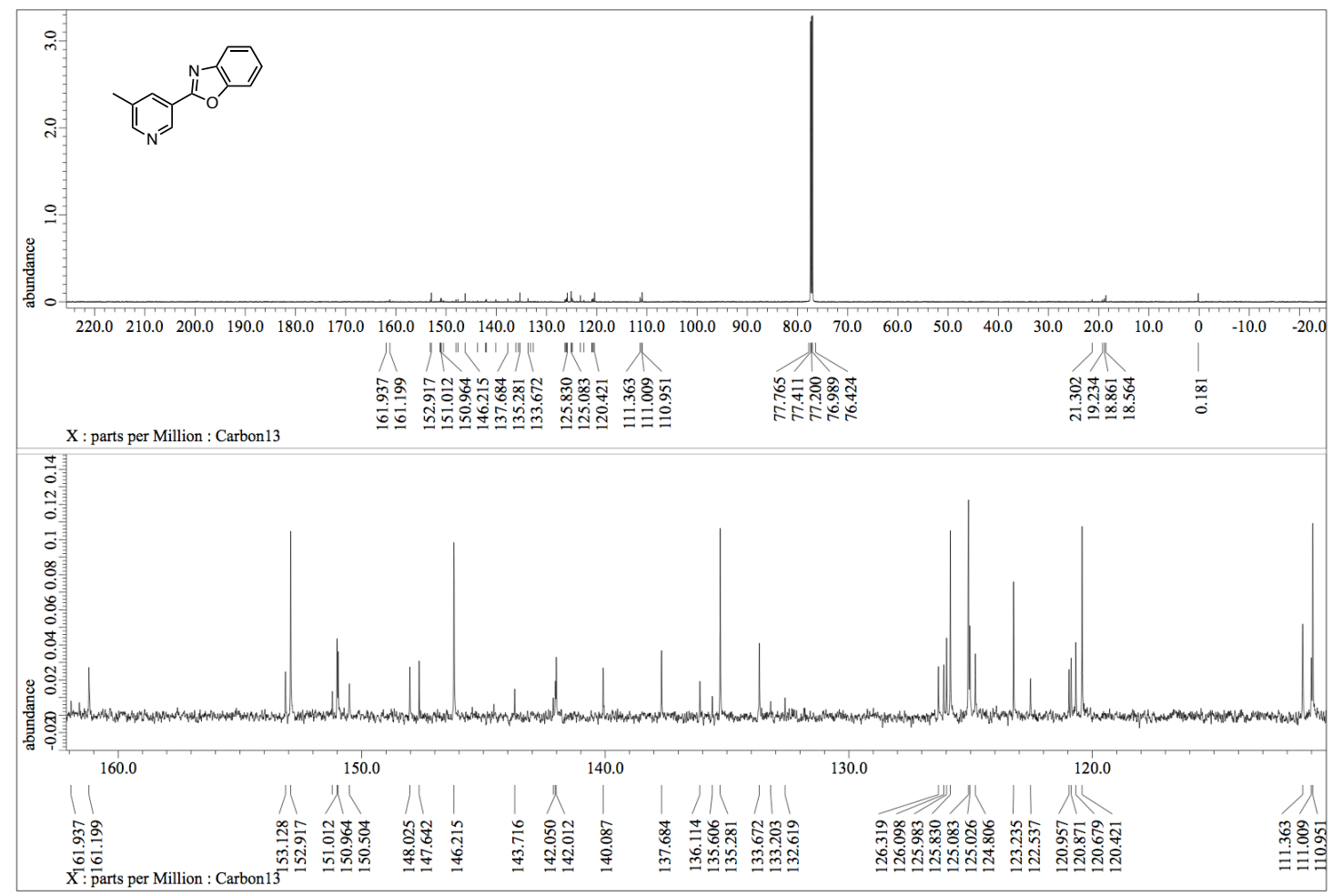

Figure S 14. ${ }^{13} \mathrm{C}$ NMR spectrum of $1 \mathrm{~g}(\mathrm{C} 2 / \mathrm{C} 4 / \mathrm{C} 5 / \mathrm{C} 6=14: 14: 51: 21)$. 


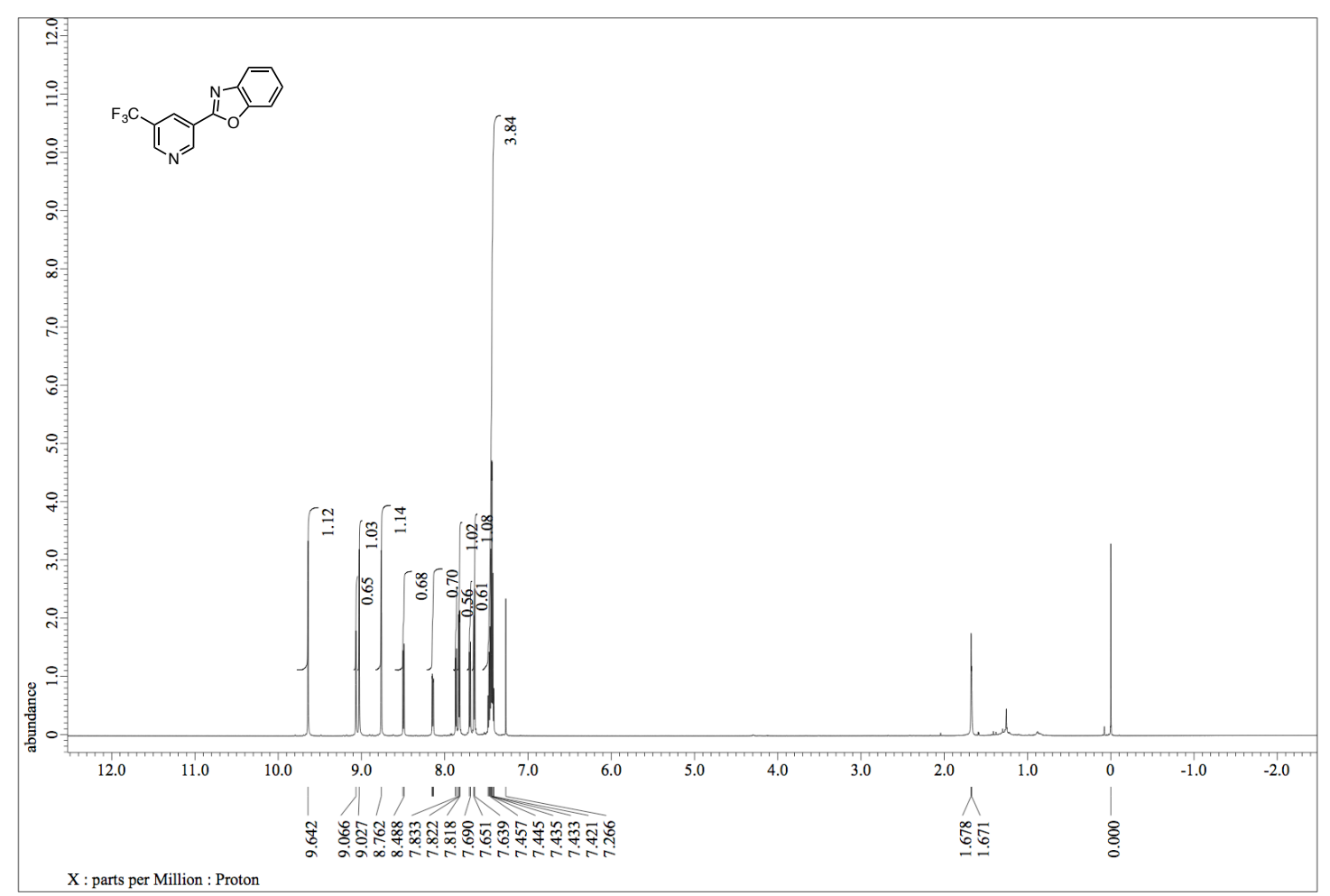

Figure S 15. ${ }^{1} \mathrm{H}$ NMR spectrum of $\mathbf{1 h}(\mathrm{C} 5 / \mathrm{C} 6=63: 37)$.

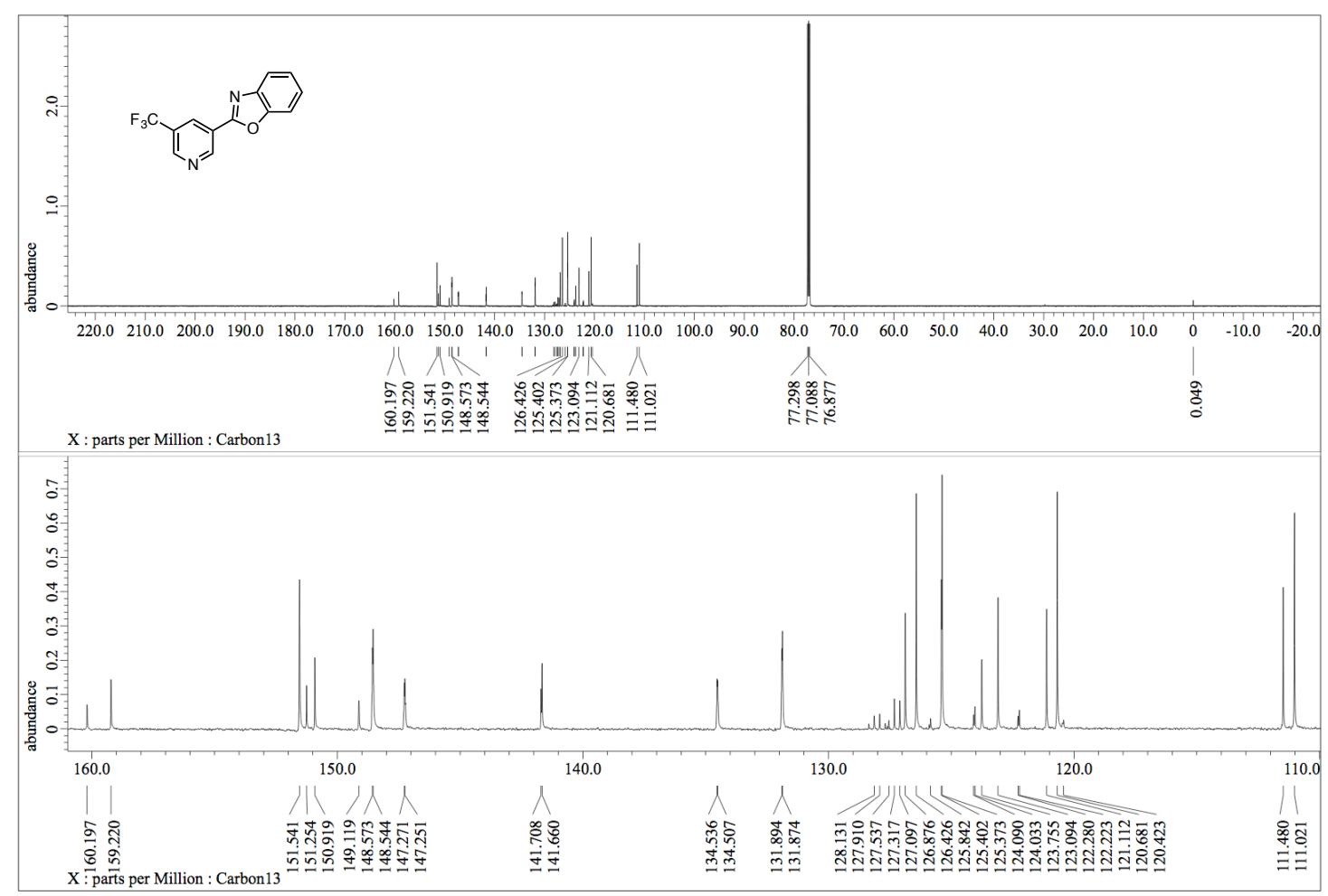

Figure S 16. ${ }^{13} \mathrm{C}$ NMR spectrum of $\mathbf{1 h}(\mathrm{C} 5 / \mathrm{C} 6=63: 37)$. 


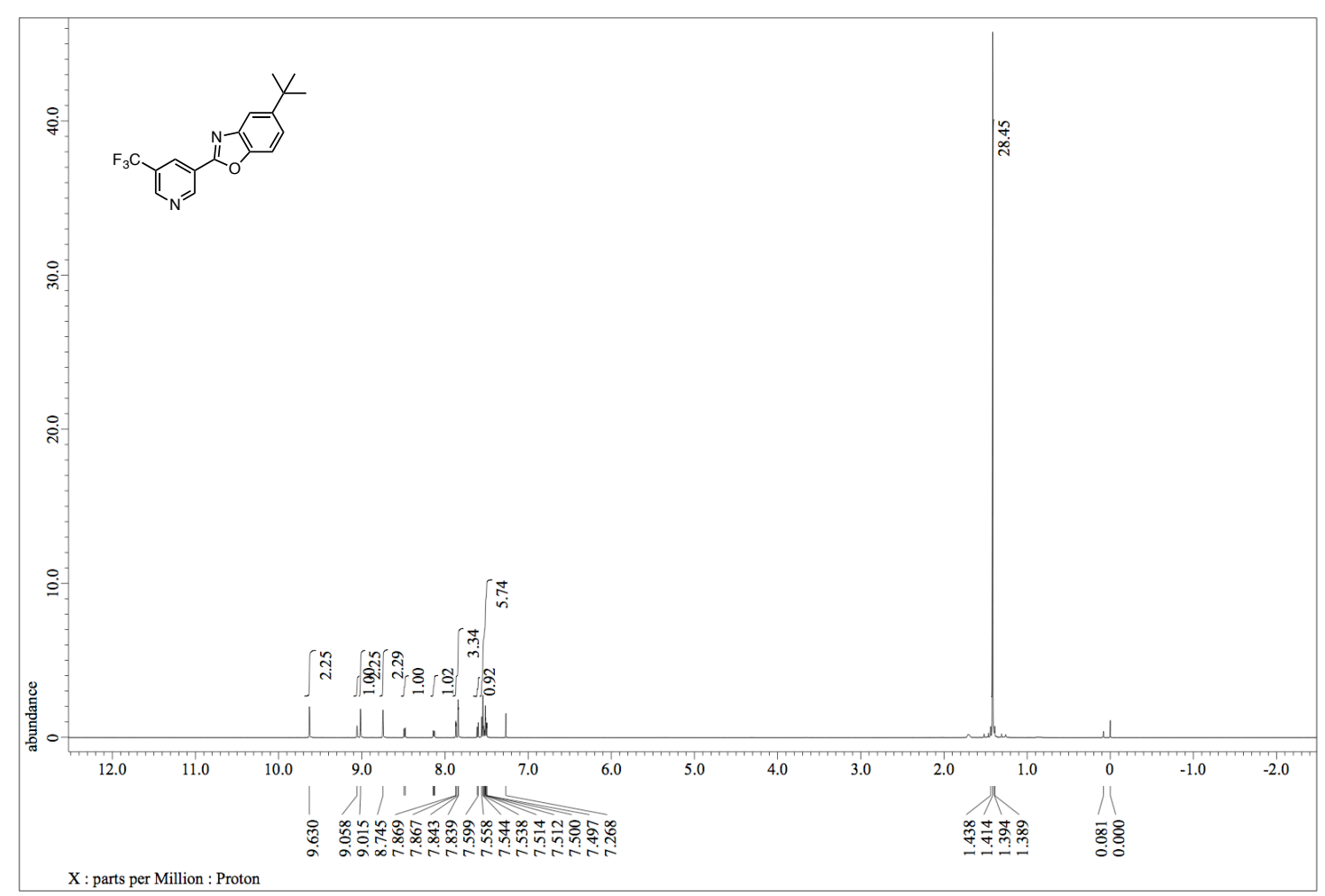

Figure S 17. ${ }^{1} \mathrm{H}$ NMR spectrum of $\mathbf{1 i}(\mathrm{C} 5 / \mathrm{C} 6=67: 33)$.

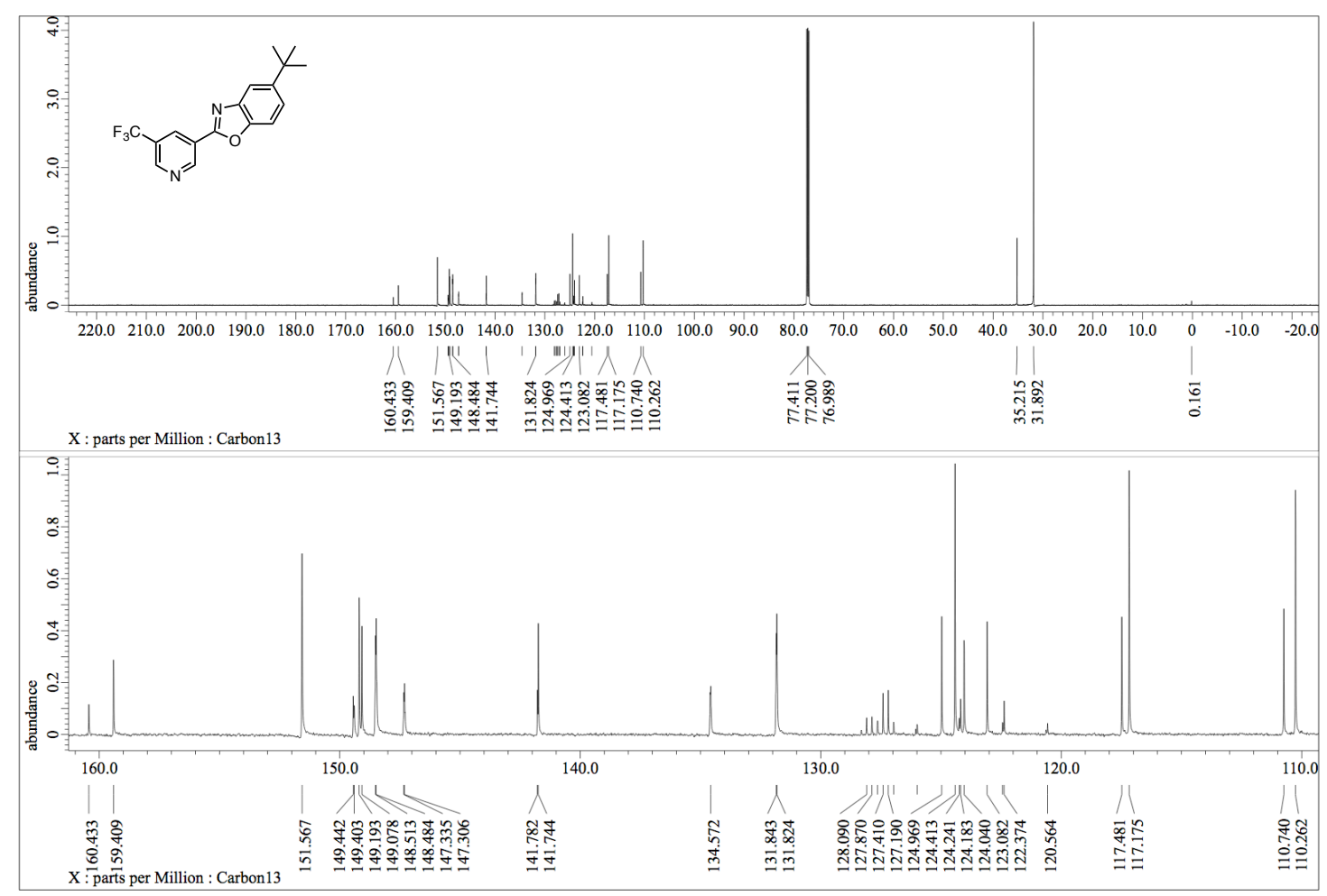

Figure S 18. ${ }^{13} \mathrm{C}$ NMR spectrum of $1 \mathbf{i}(\mathrm{C} 5 / \mathrm{C} 6=67: 33)$. 


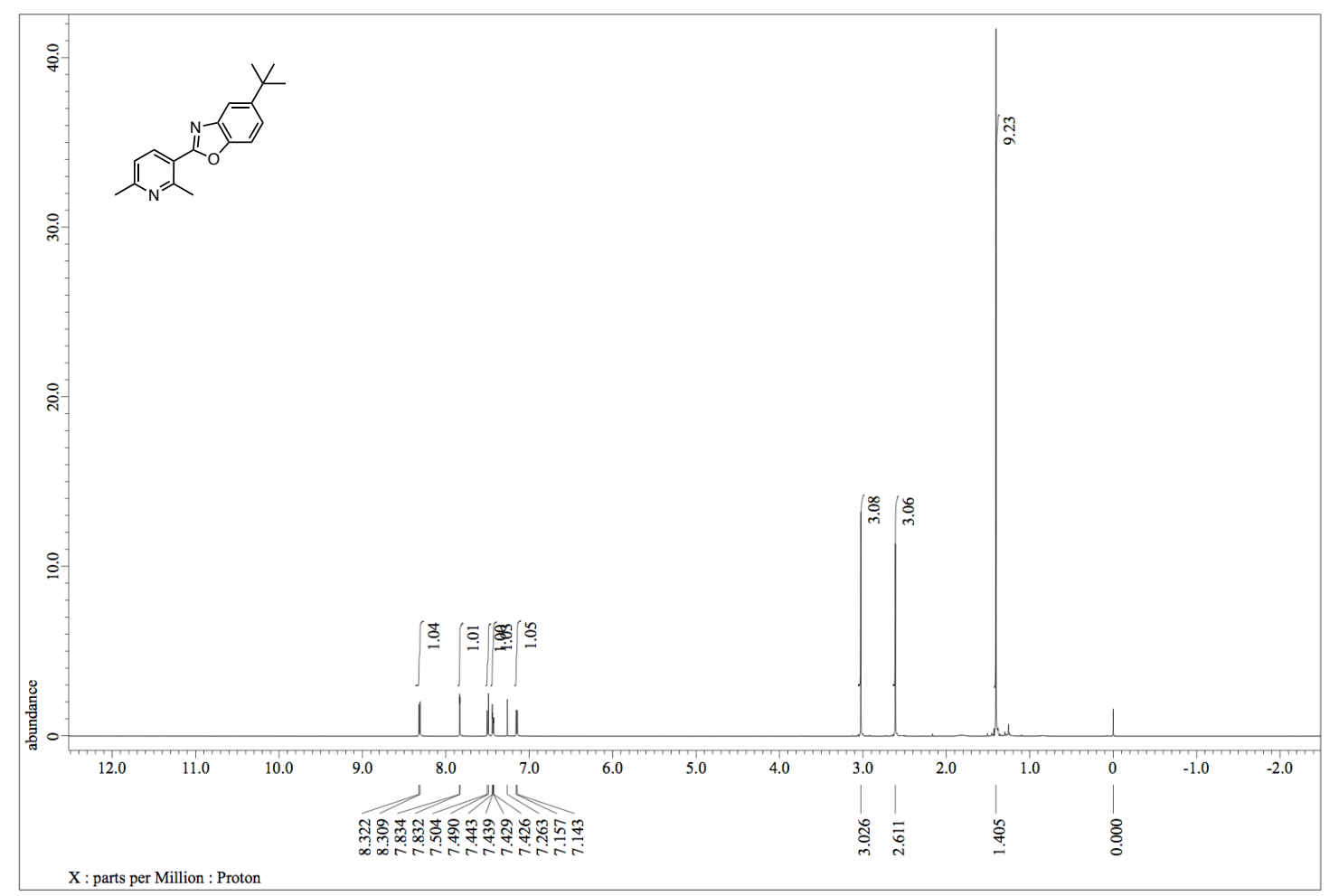

Figure $\mathbf{S}$ 19. ${ }^{1} \mathrm{H}$ NMR spectrum of $\mathbf{1 j}$.

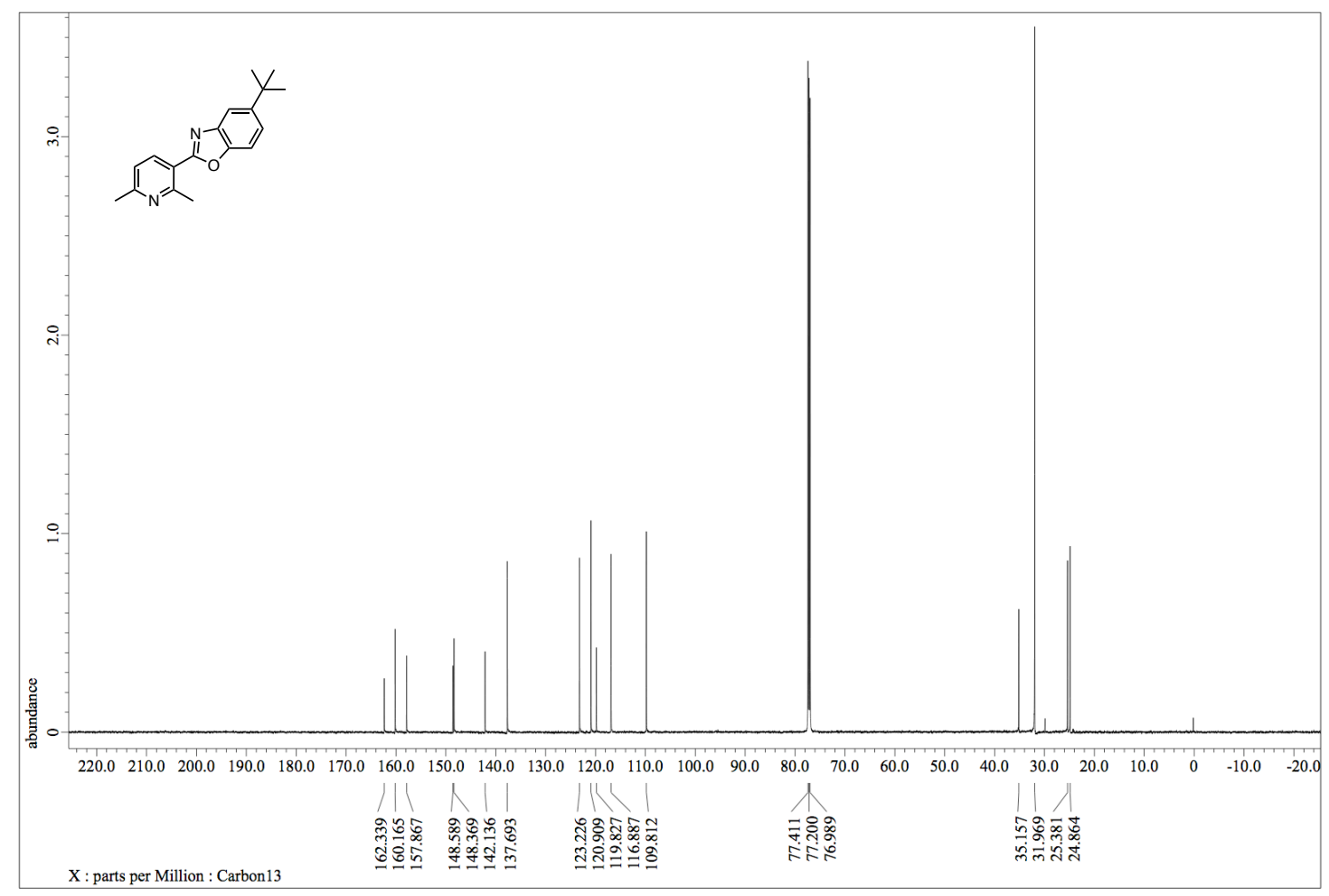

Figure S 20. ${ }^{13} \mathrm{C}$ NMR spectrum of $\mathbf{1 j}$. 


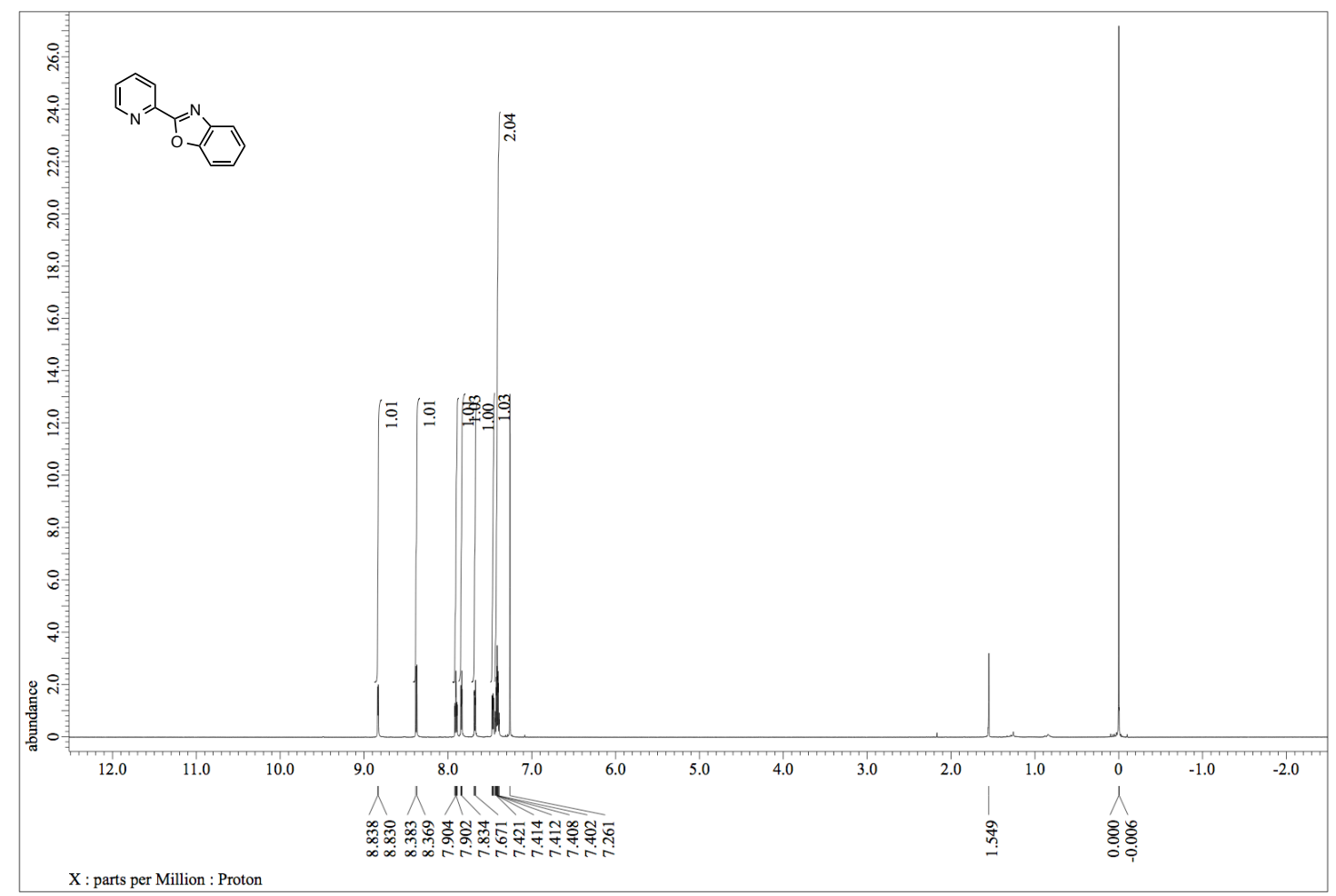

Figure S 21. ${ }^{1} \mathrm{H}$ NMR spectrum of $\mathbf{2 a}$.

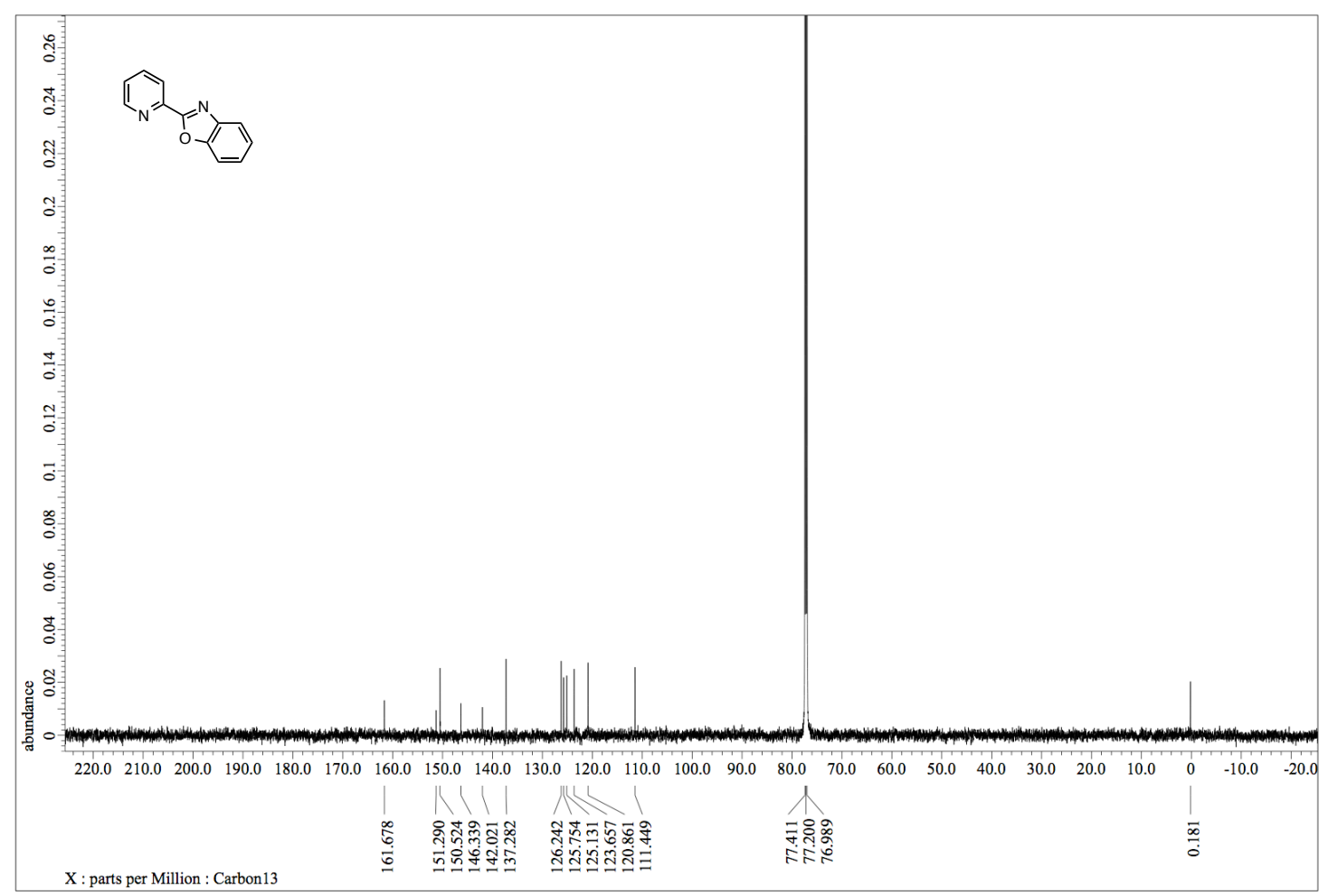

Figure S 22. ${ }^{13} \mathrm{C}$ NMR spectrum of $\mathbf{2 a}$. 


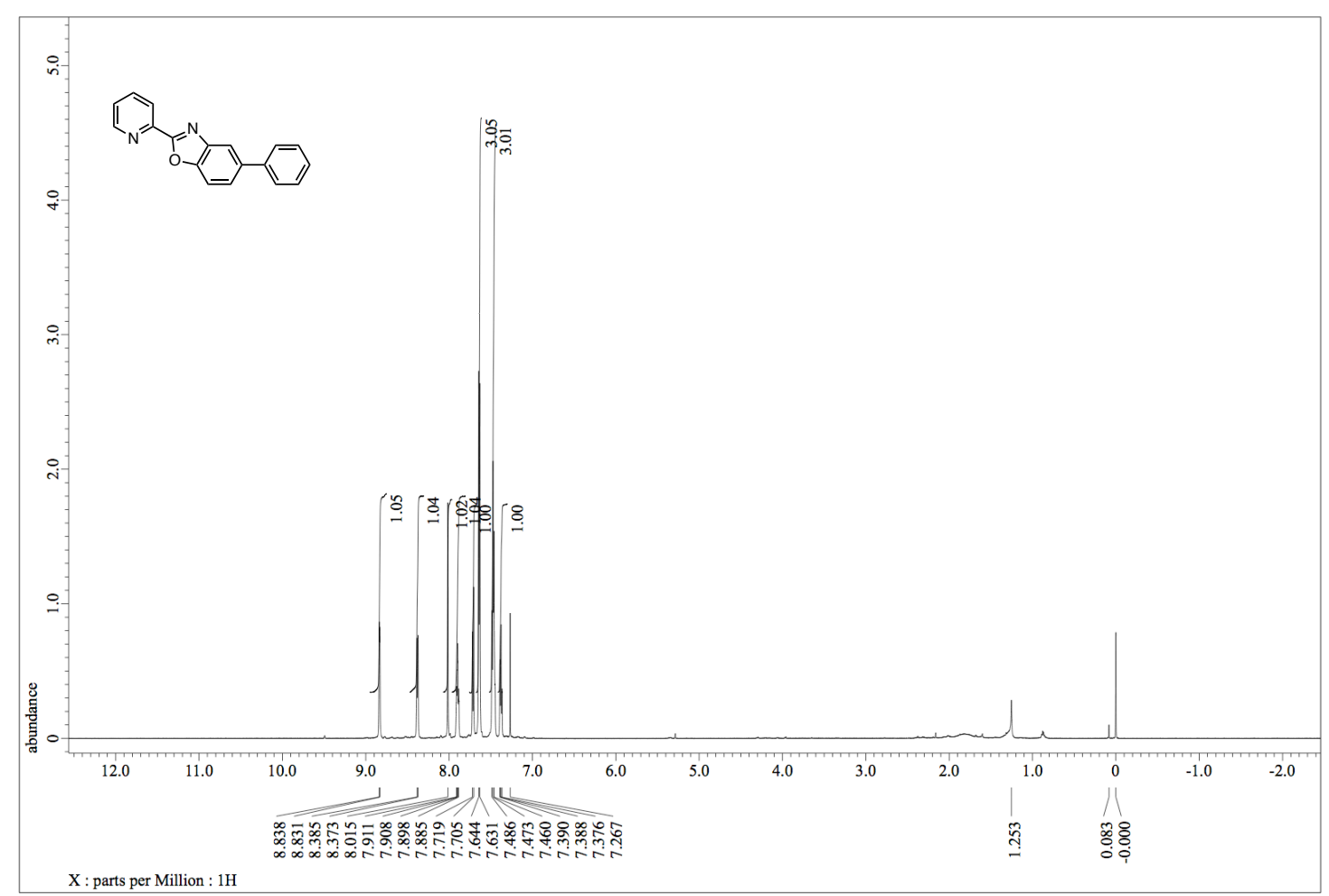

Figure $\mathbf{S} 23 .{ }^{1} \mathrm{H}$ NMR spectrum of $\mathbf{2 b}$.

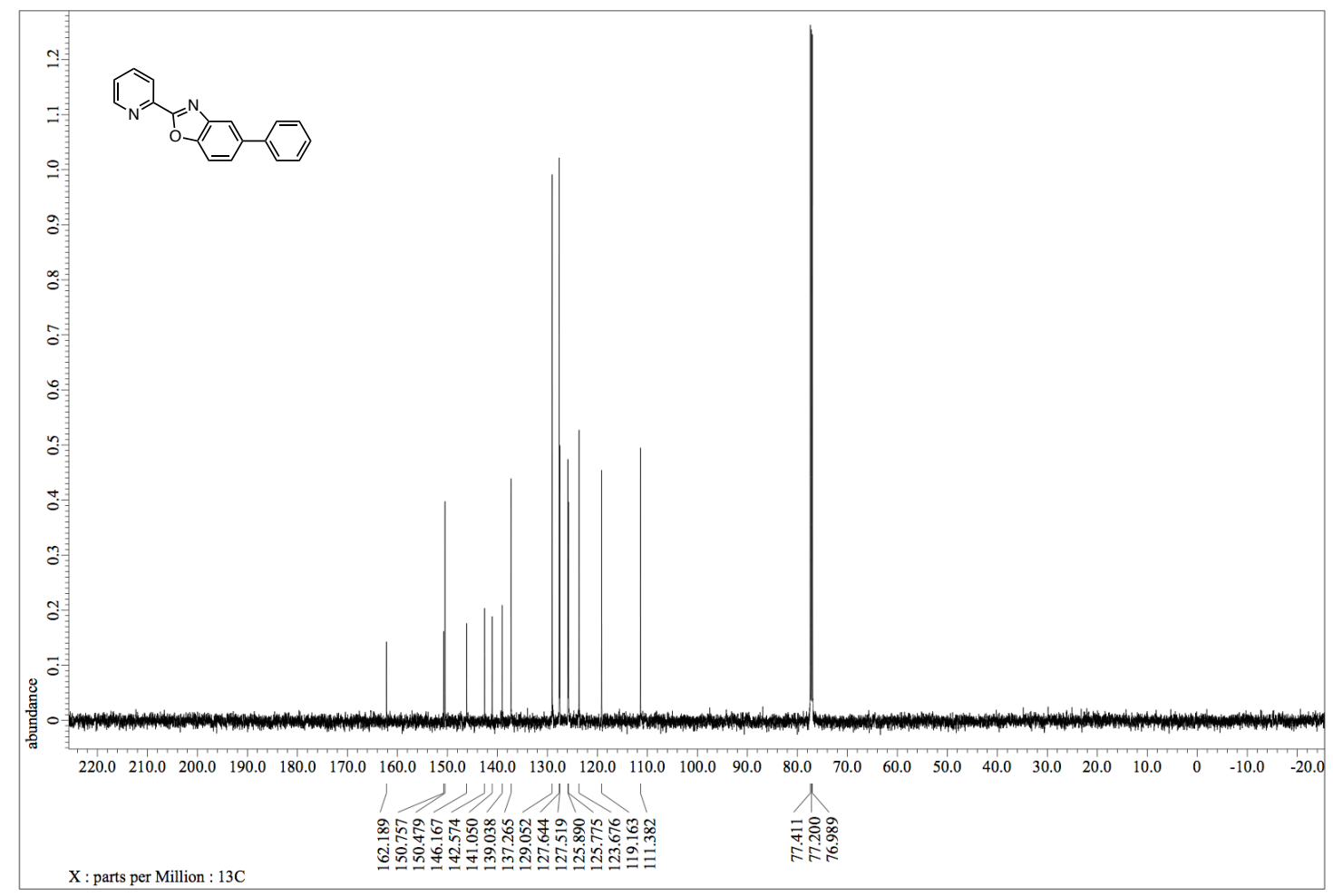

Figure S 24. ${ }^{13} \mathrm{C}$ NMR spectrum of $\mathbf{2 b}$. 


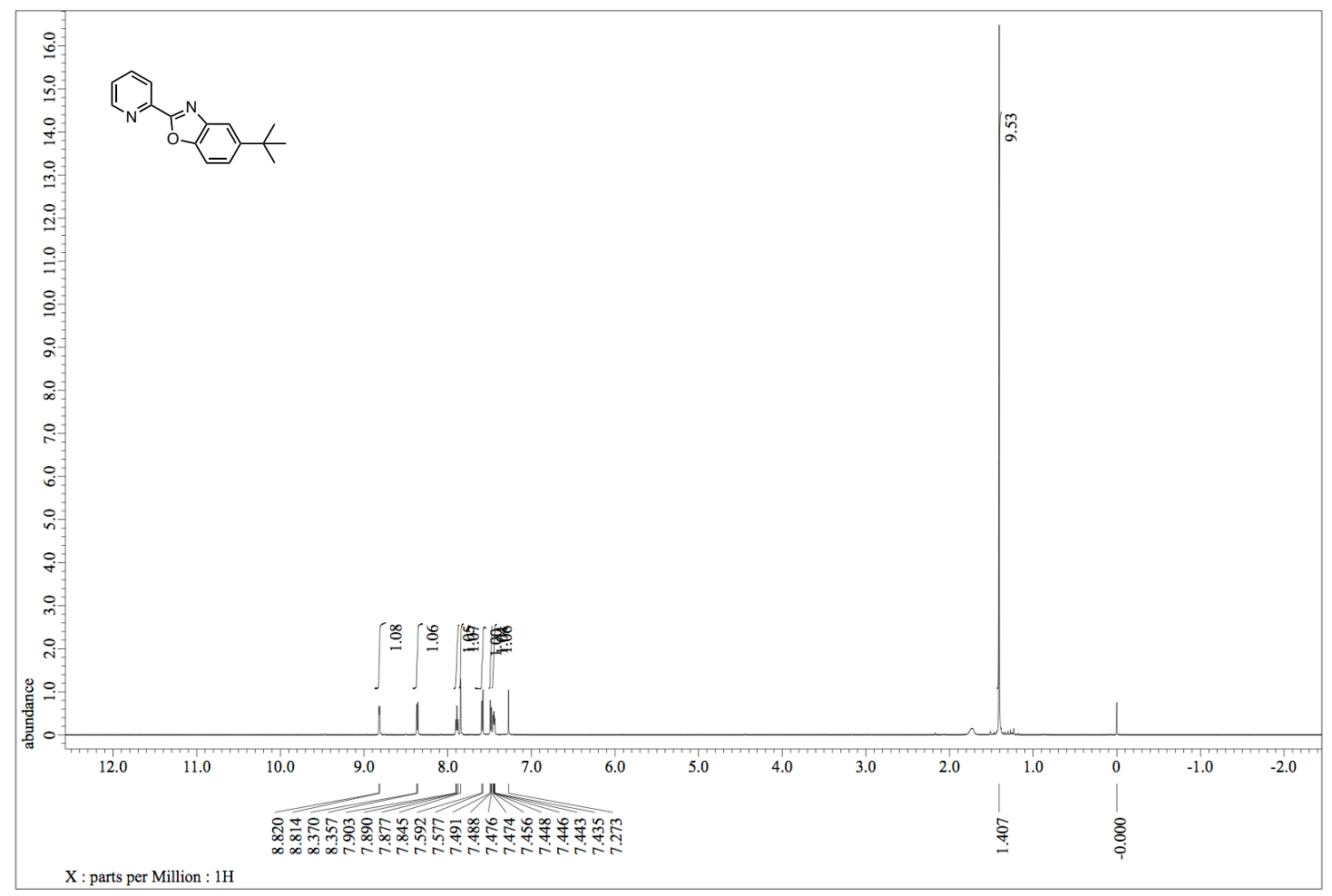

Figure S 25. ${ }^{1} \mathrm{H}$ NMR spectrum of $\mathbf{2 c}$.

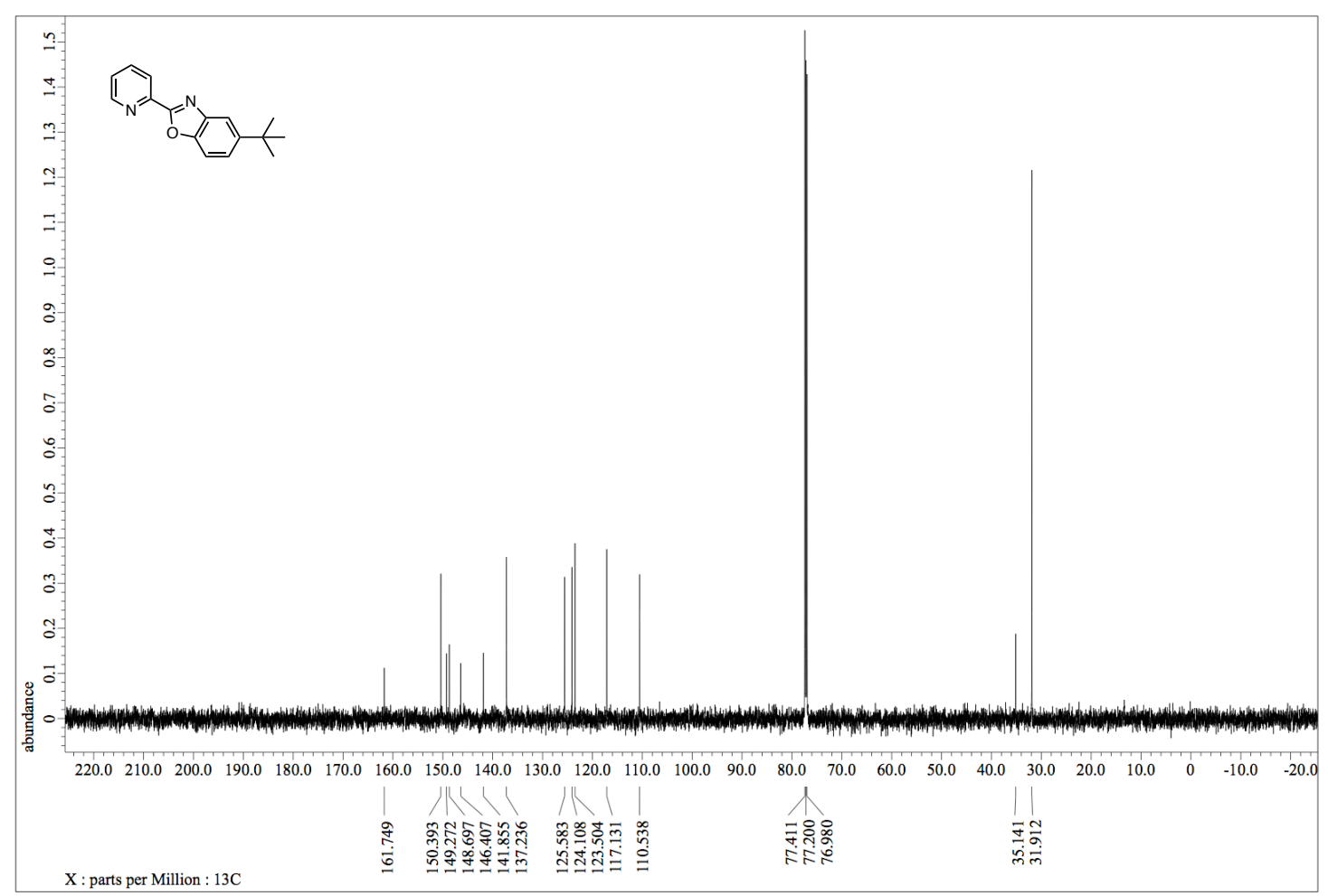

Figure S 26. ${ }^{13} \mathrm{C}$ NMR spectrum of $2 \mathrm{c}$. 


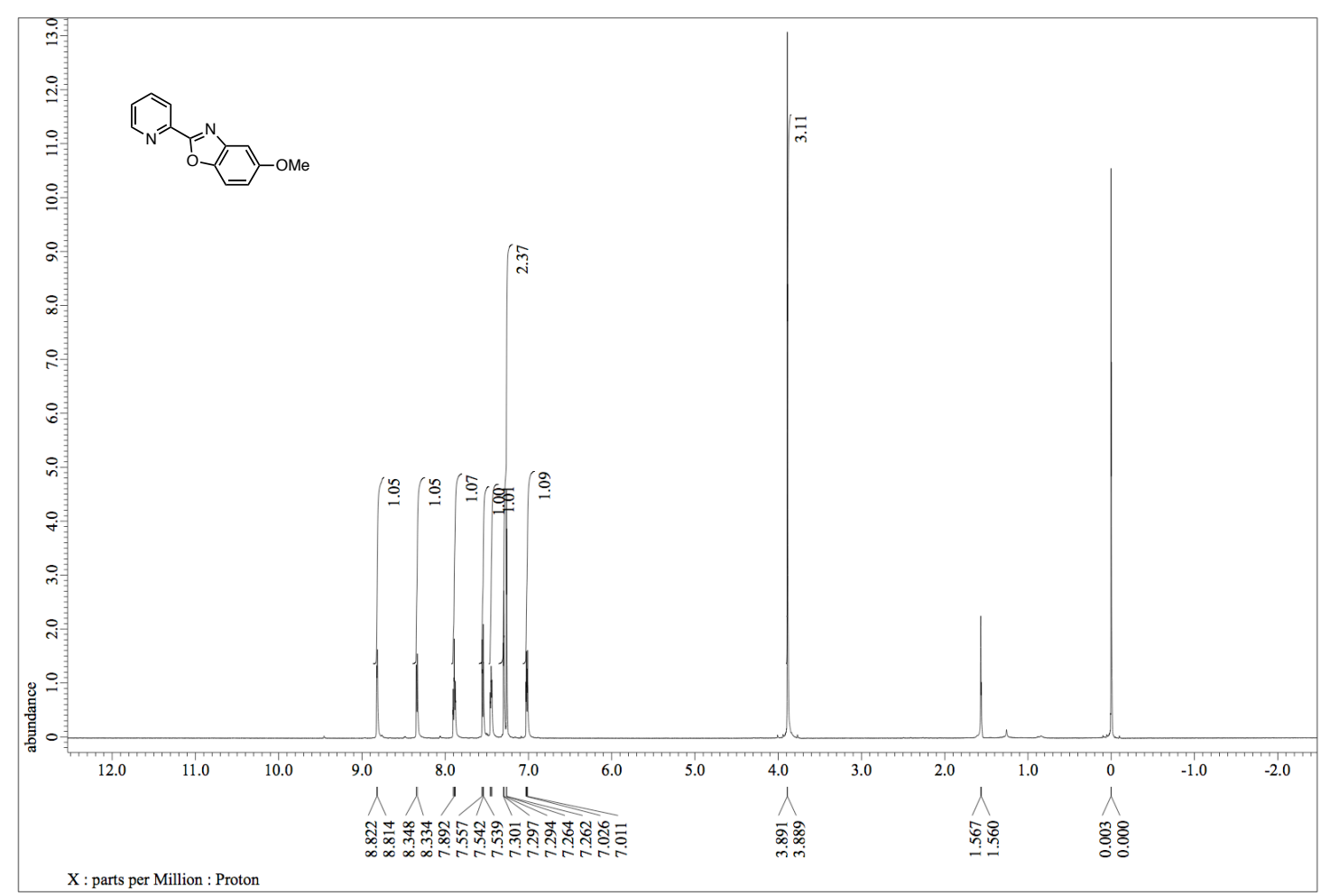

Figure $\mathbf{S} 27 .{ }^{1} \mathrm{H}$ NMR spectrum of $\mathbf{2 d}$.

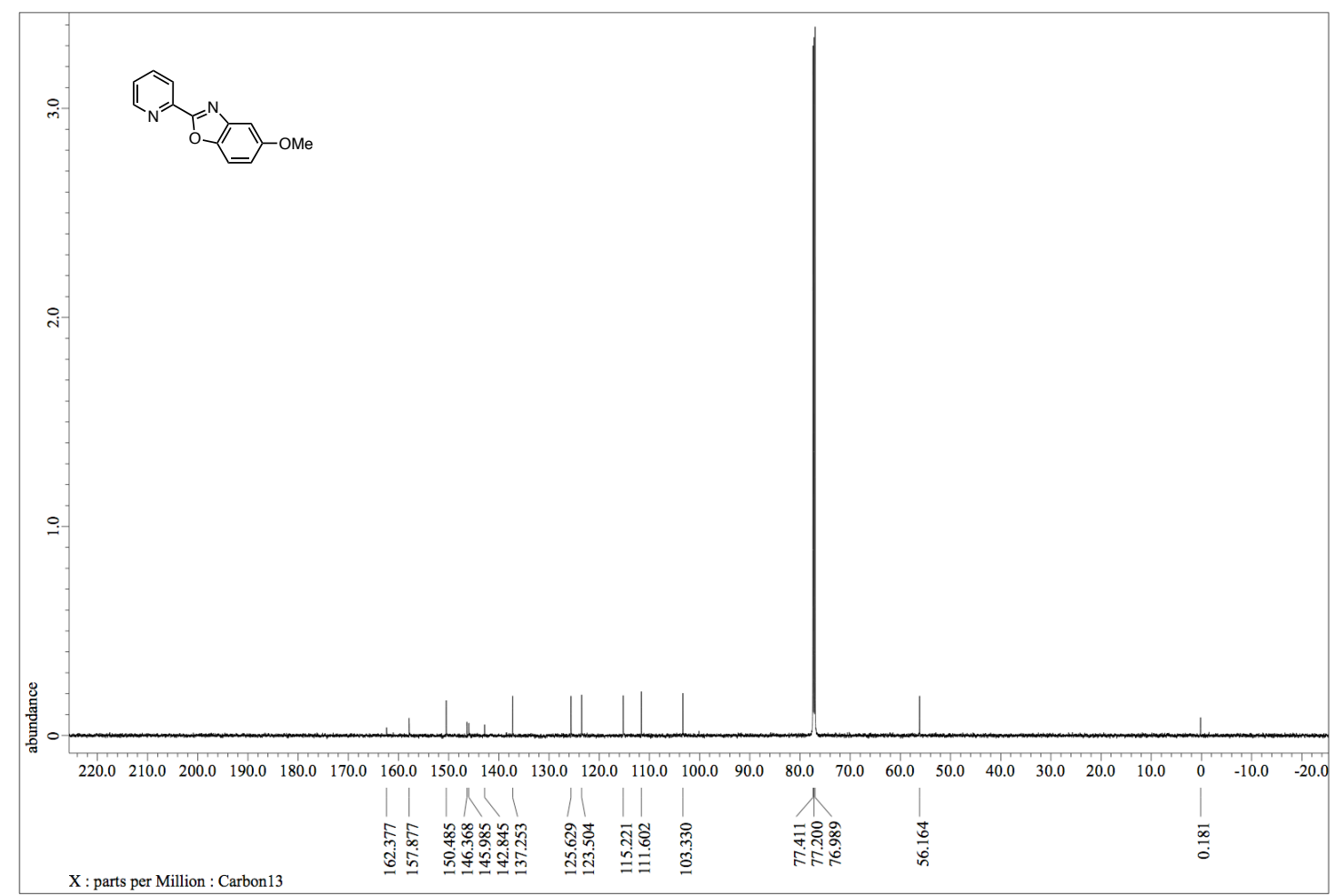

Figure S 28. ${ }^{13} \mathrm{C}$ NMR spectrum of $\mathbf{2 d}$. 


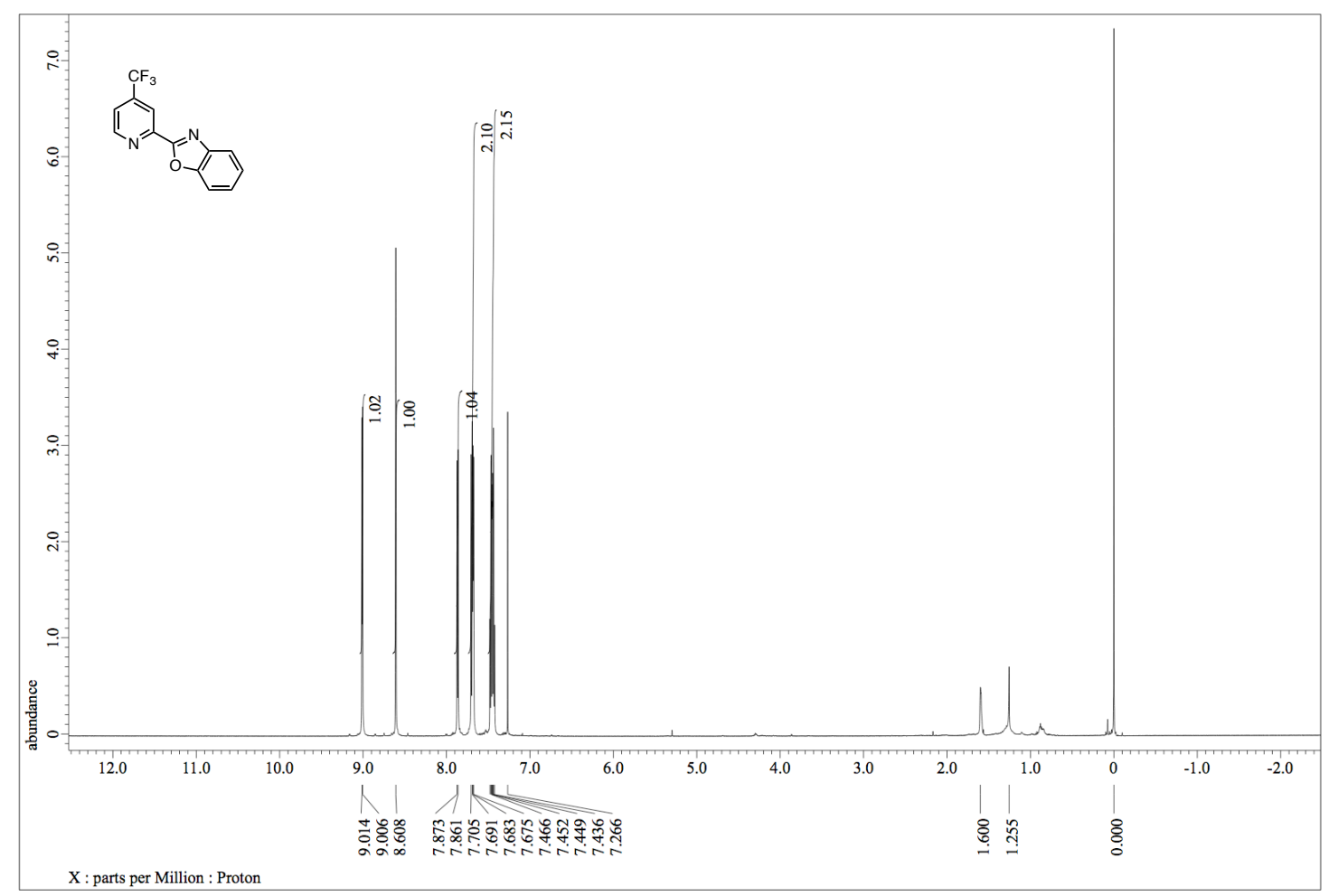

Figure S 29. ${ }^{1} \mathrm{H}$ NMR spectrum of $2 \mathbf{e}$.

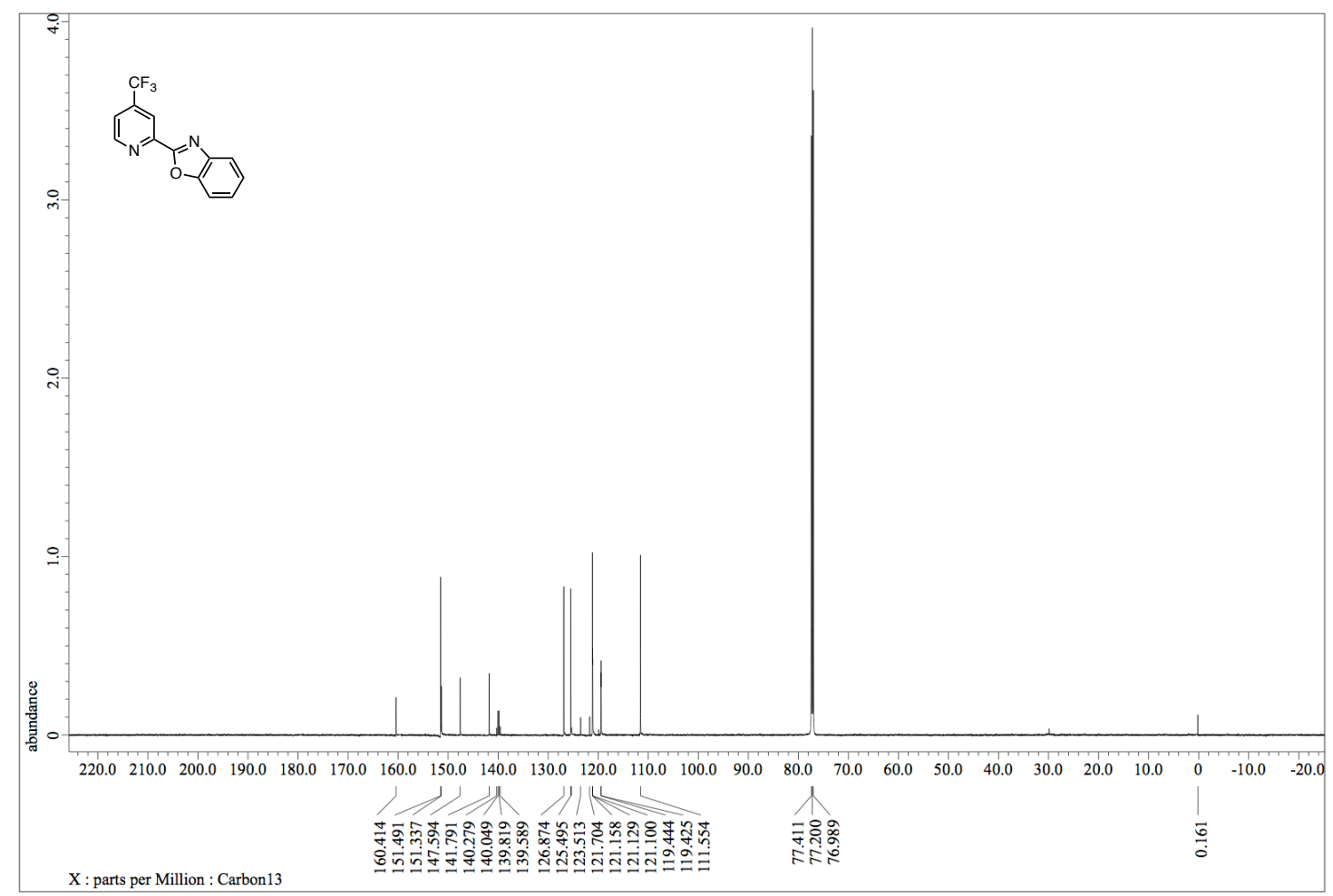

Figure S 30. ${ }^{13} \mathrm{C}$ NMR spectrum of $2 \mathrm{e}$. 
Regiodivergent Cross-Dehydrogenative Coupling of Pyridines and Benzoxazoles: Discovery of Organic Halides as Regio-Switching Oxidants Shuya Yamada, Kei Murakami, Kenichiro Itami

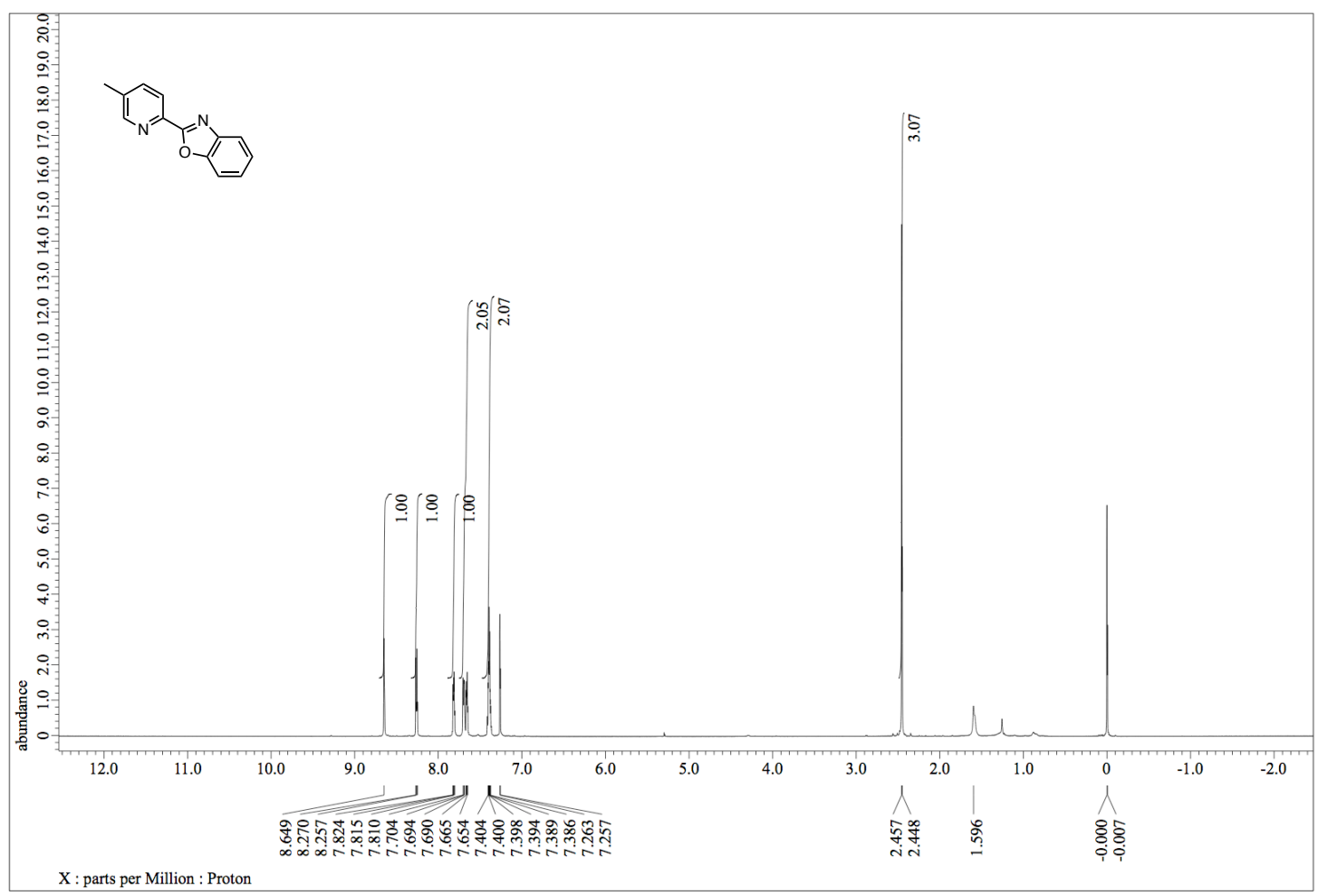

Figure S 31. ${ }^{1} \mathrm{H}$ NMR spectrum of $\mathbf{2 f}$.

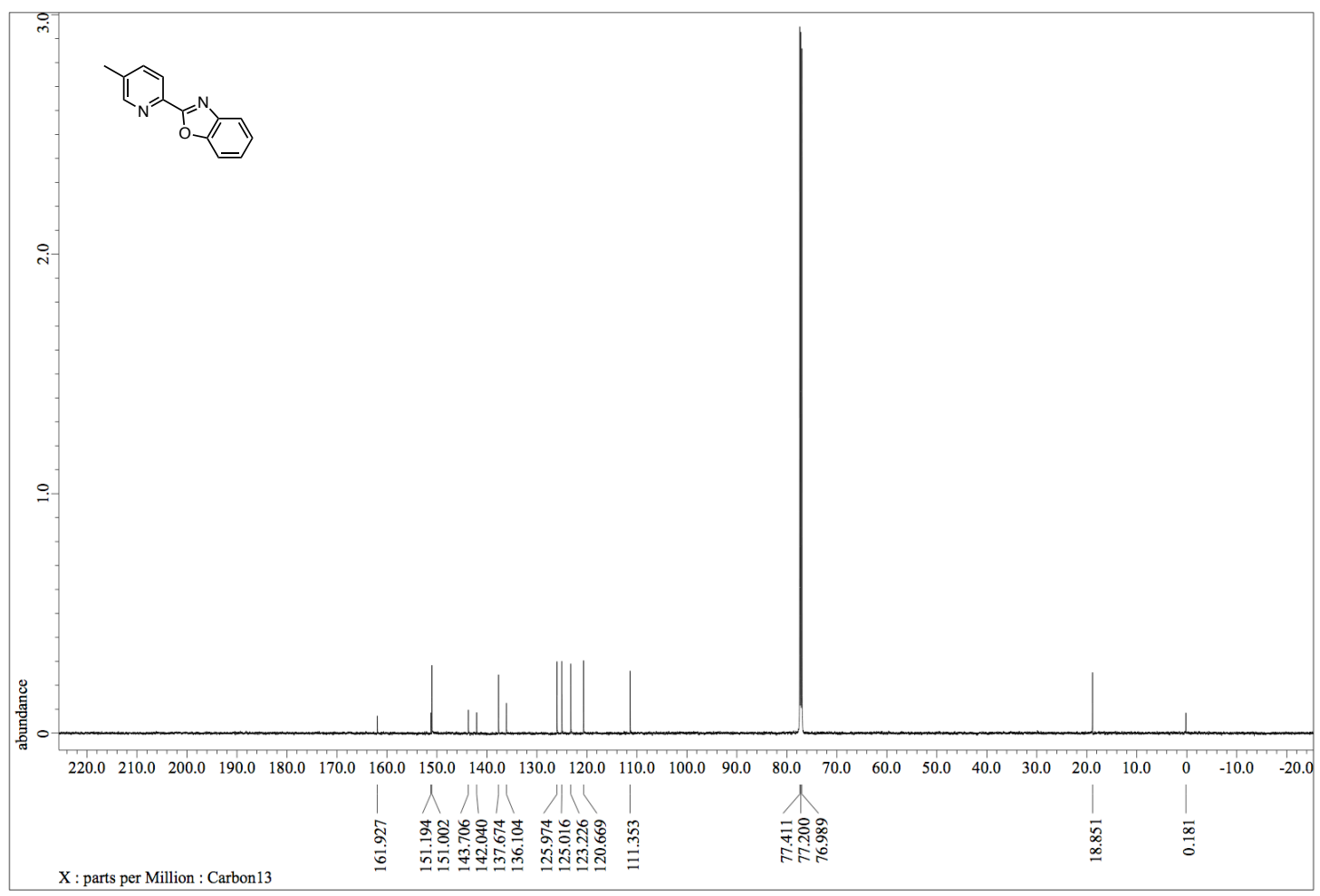

Figure S 32. ${ }^{13} \mathrm{C}$ NMR spectrum of $\mathbf{2 f}$.

S34 
Regiodivergent Cross-Dehydrogenative Coupling of Pyridines and Benzoxazoles: Discovery of Organic Halides as Regio-Switching Oxidants Shuya Yamada, Kei Murakami, Kenichiro Itami

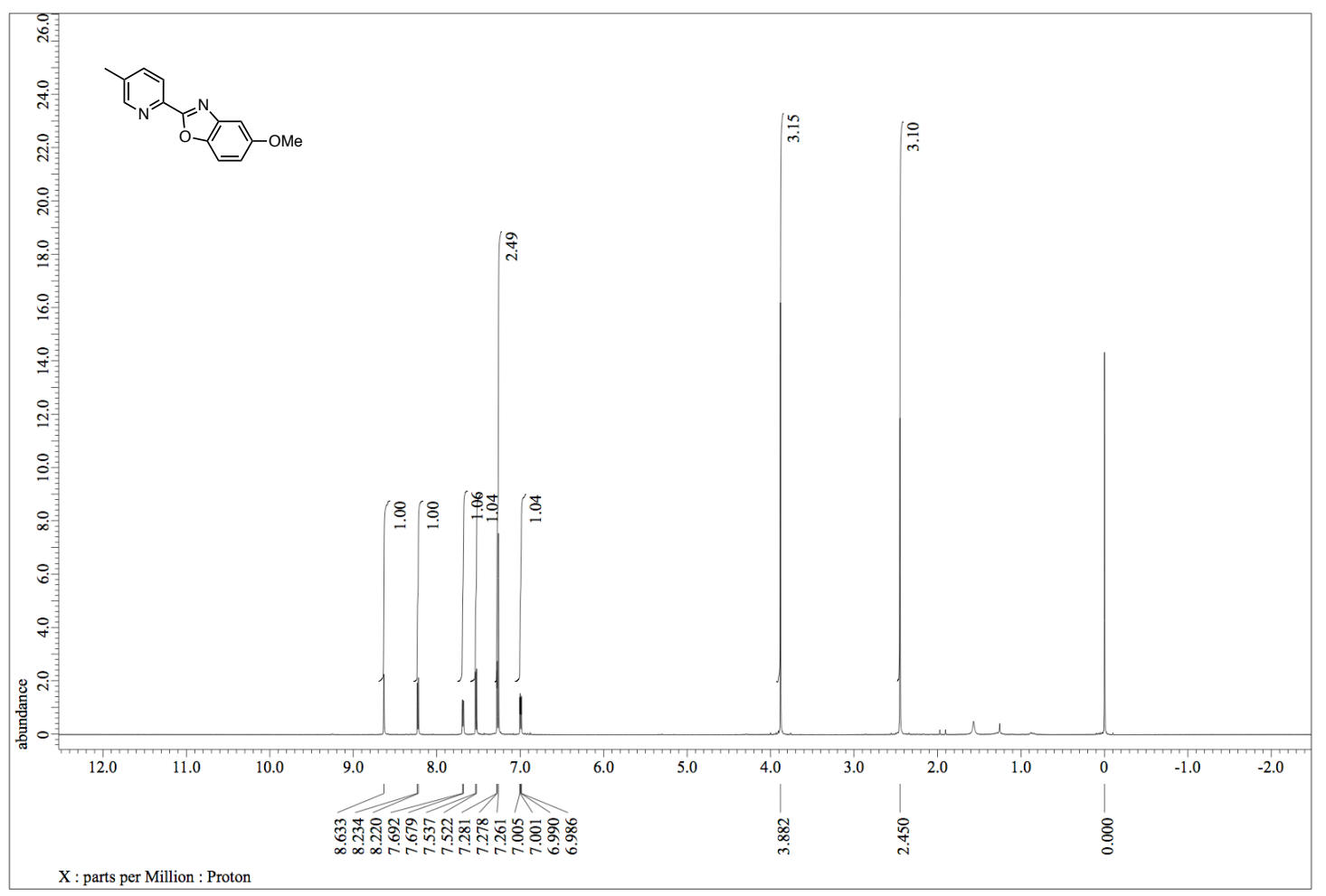

Figure S 33. ${ }^{1} \mathrm{H}$ NMR spectrum of $\mathbf{2 g}$.

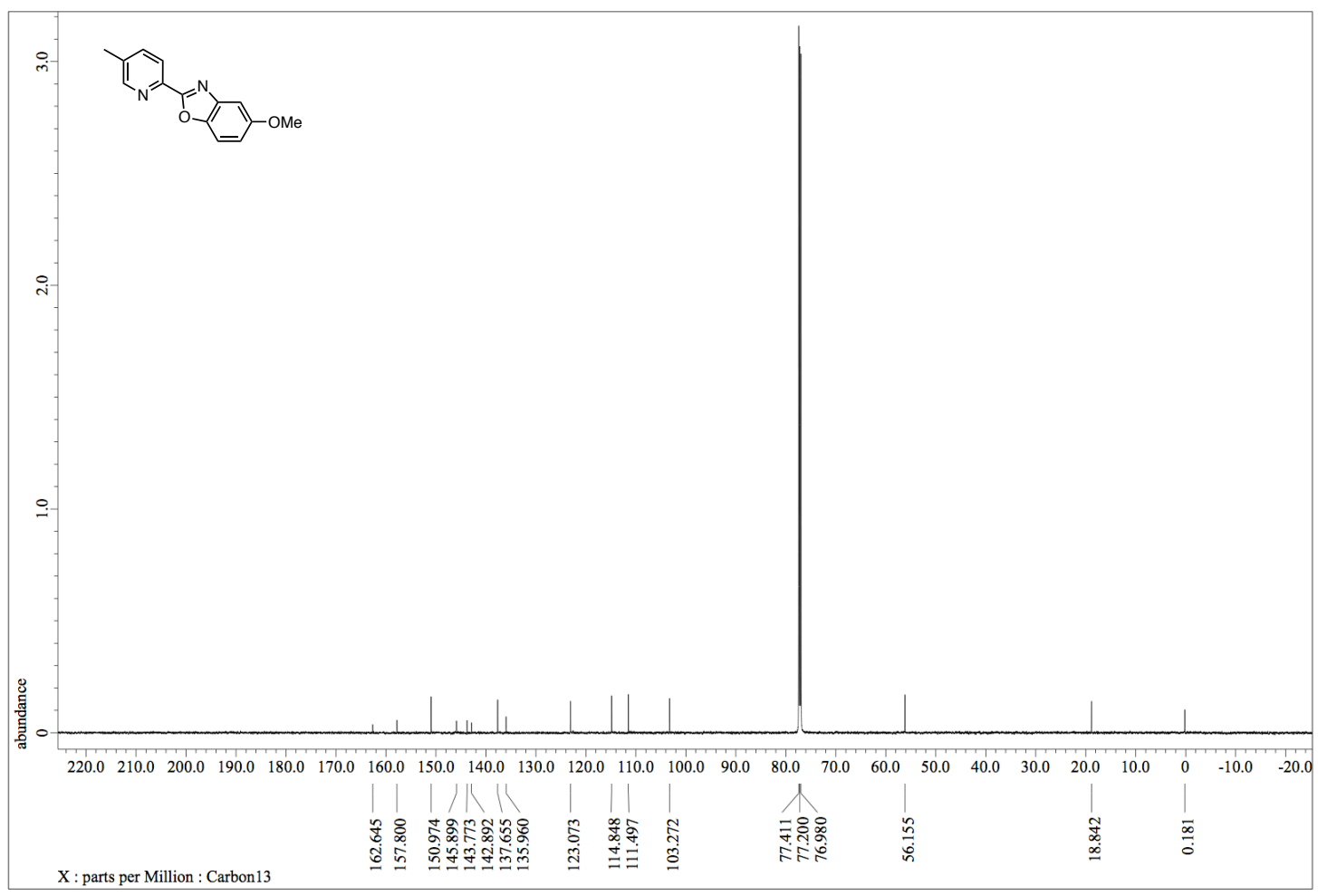

Figure S 34. ${ }^{13} \mathrm{C}$ NMR spectrum of $\mathbf{2 g}$.

S35 


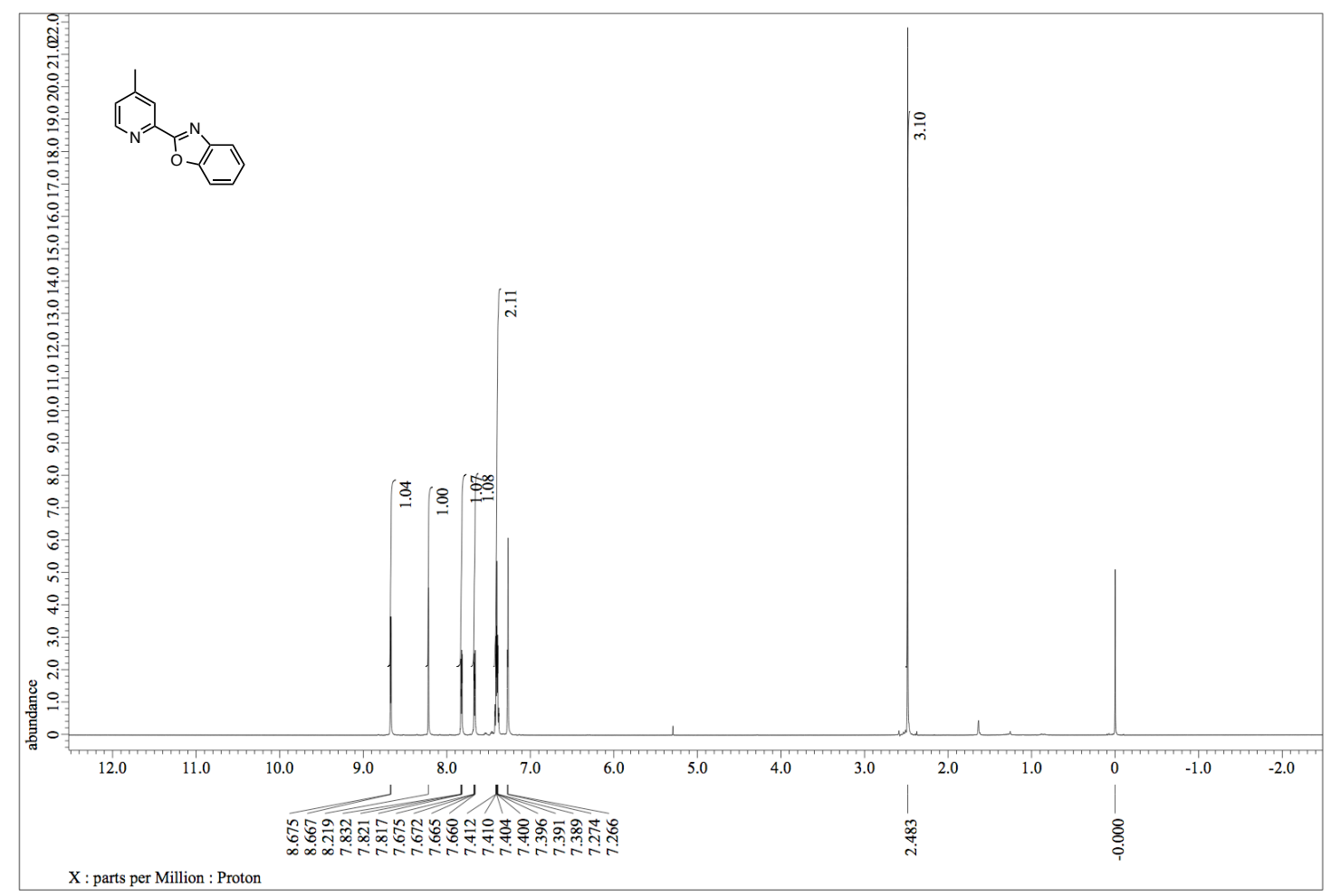

Figure S 35. ${ }^{1} \mathrm{H}$ NMR spectrum of authentic 1f-C2.

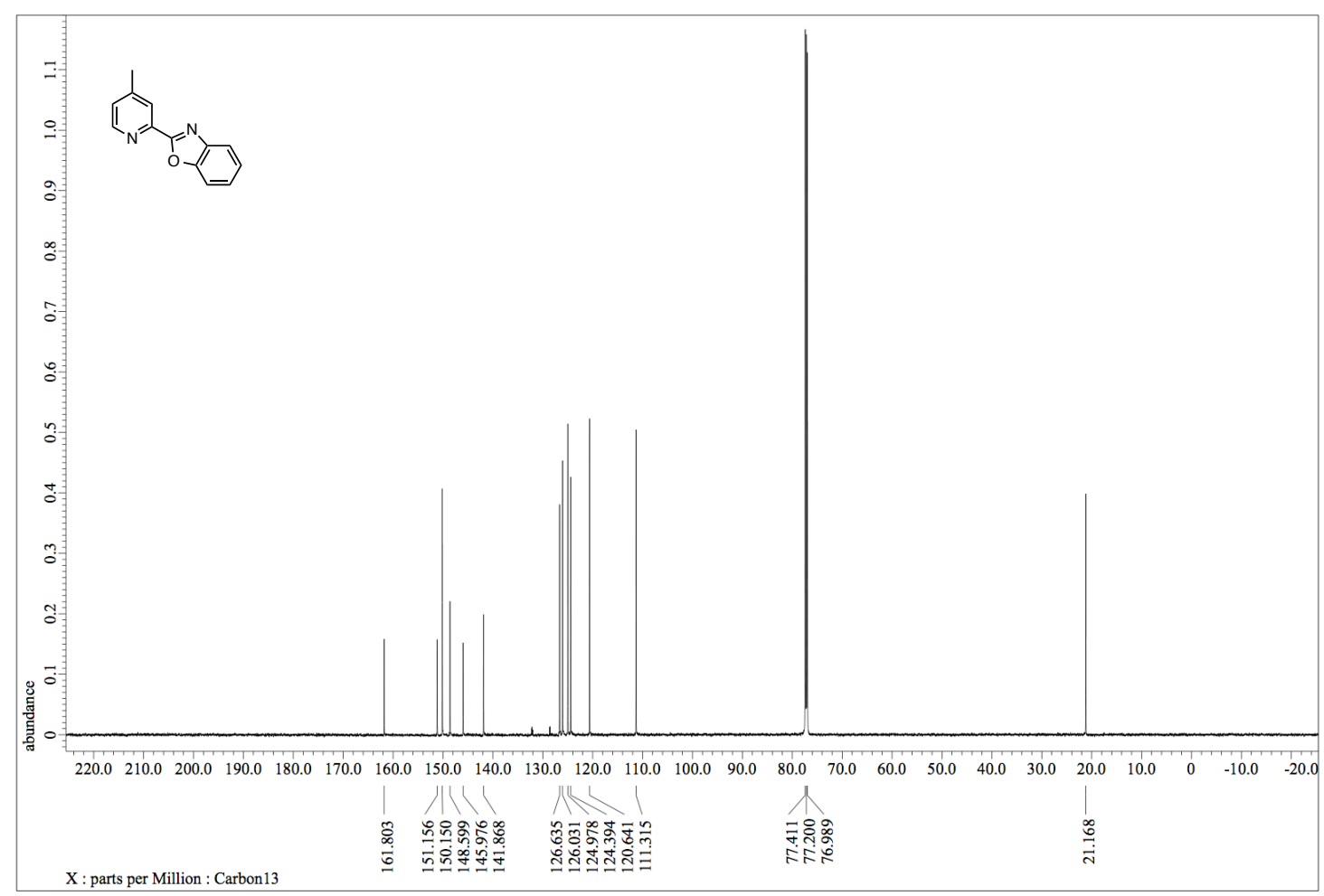

Figure S 36. ${ }^{13} \mathrm{C}$ NMR spectrum of authentic 1f-C2. 


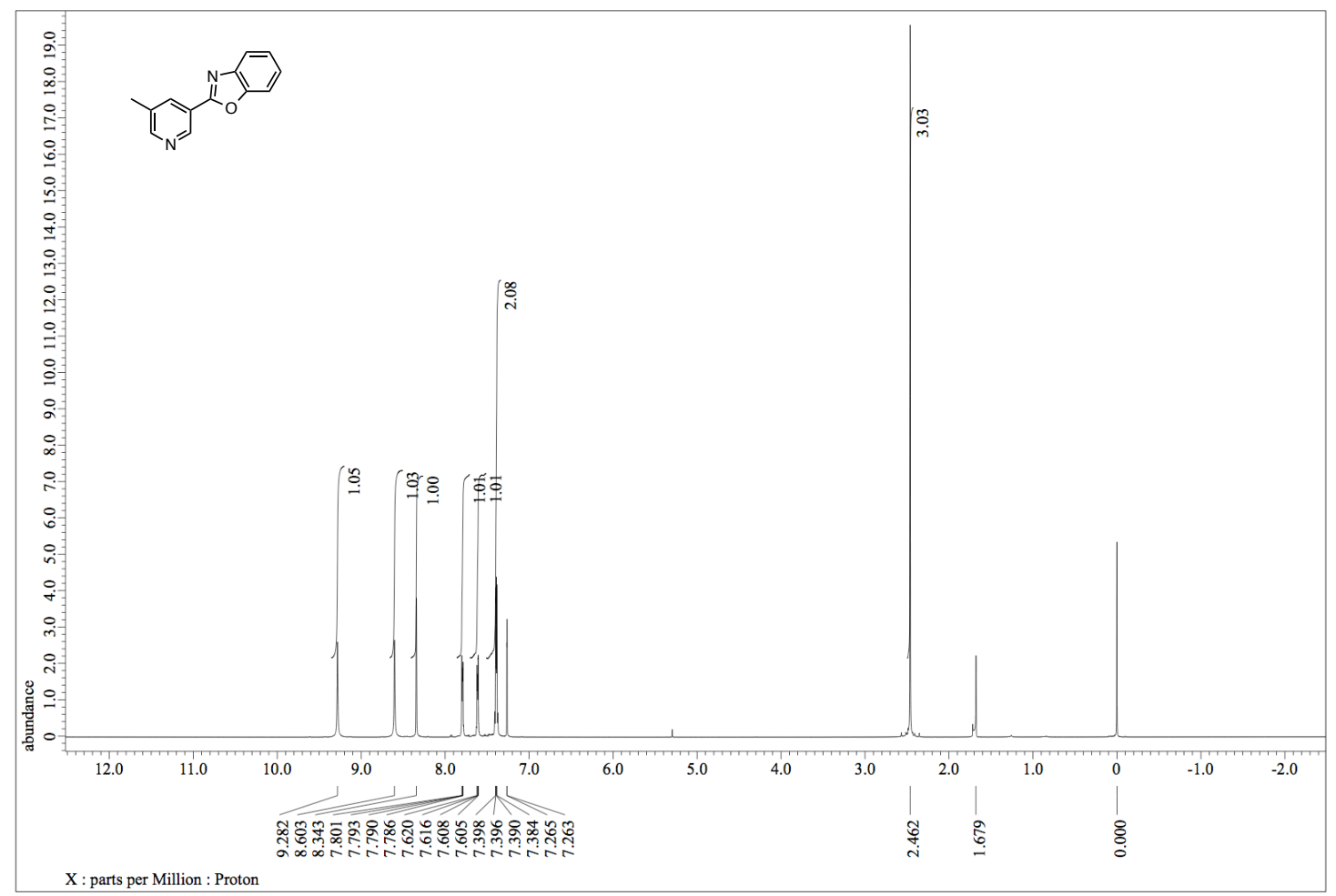

Figure S 37. ${ }^{1} \mathrm{H}$ NMR spectrum of authentic 1g-C5.

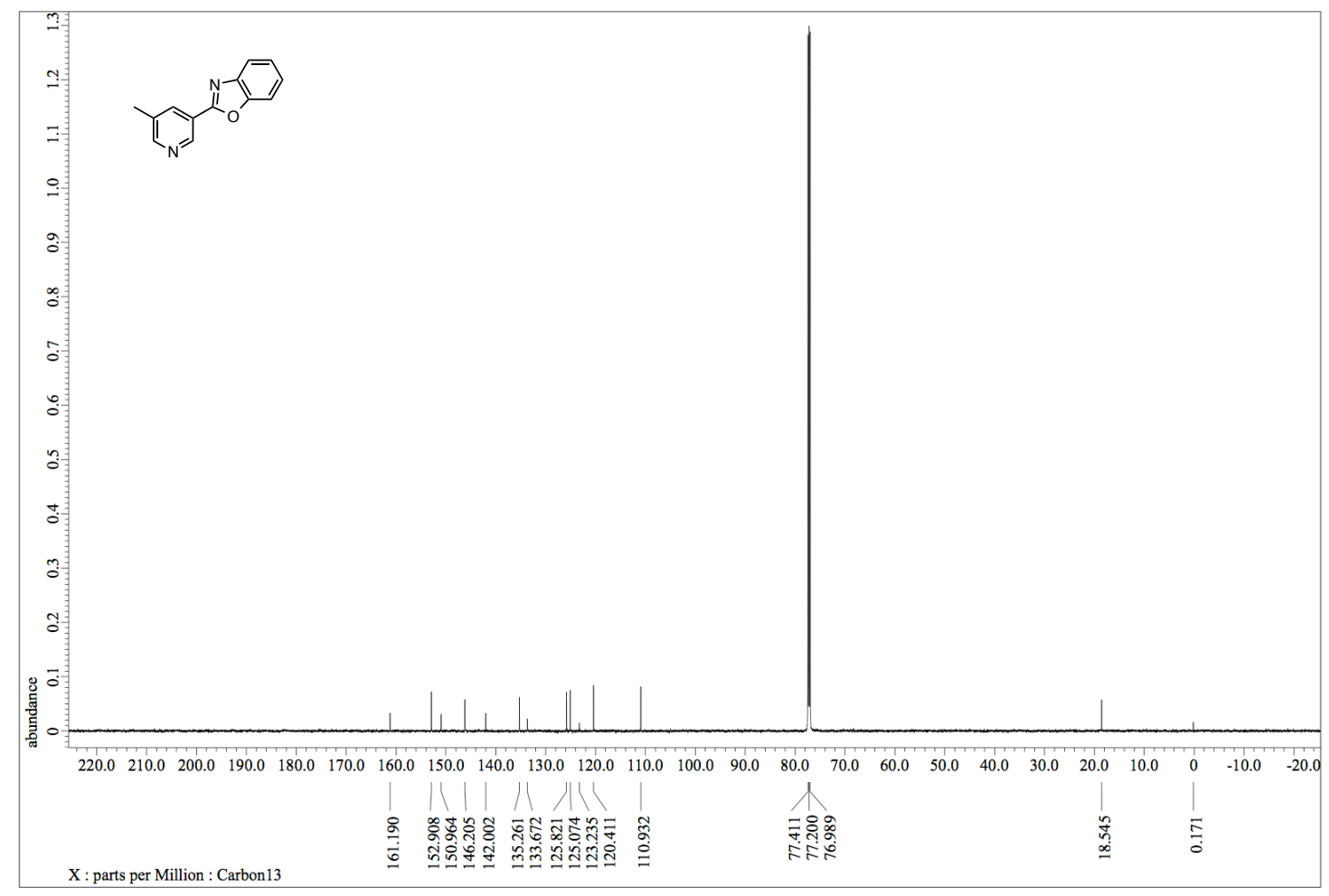

Figure S 38. ${ }^{13} \mathrm{C}$ NMR spectrum of authentic 1g-C5. 


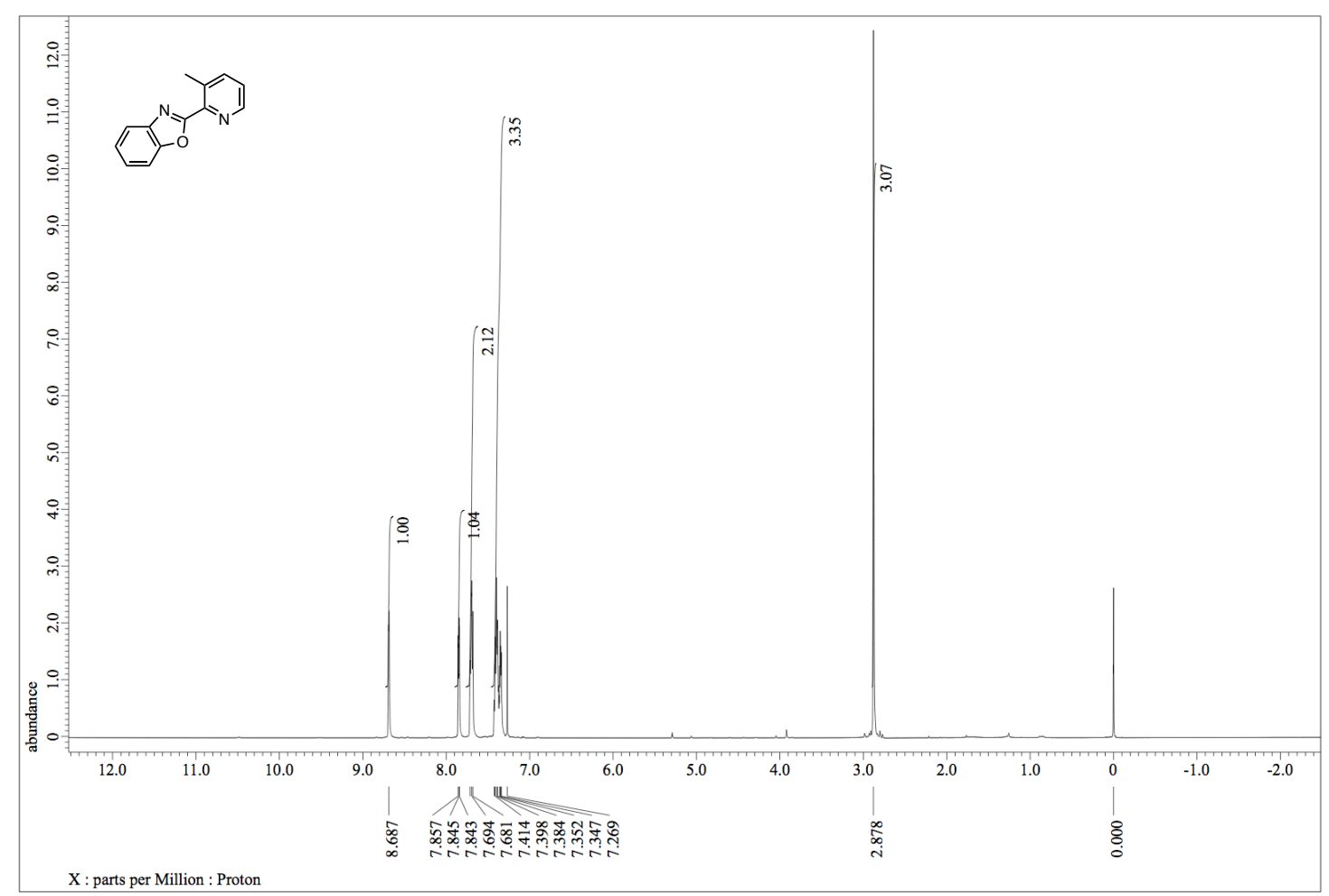

Figure S 39. ${ }^{1} \mathrm{H}$ NMR spectrum of authentic 1g-C2.

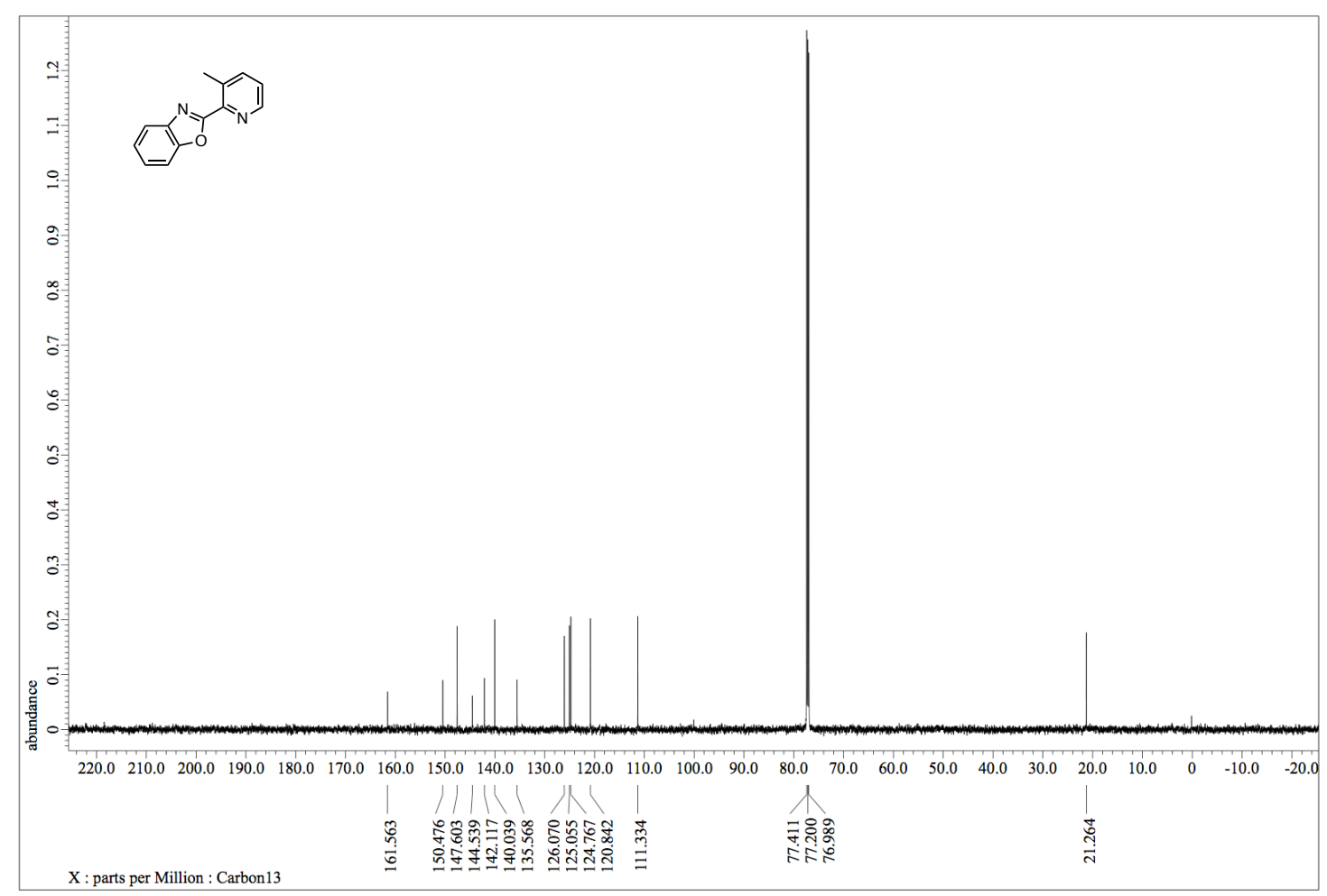

Figure S 40. ${ }^{13} \mathrm{C}$ NMR spectrum of authentic 1g-C2. 


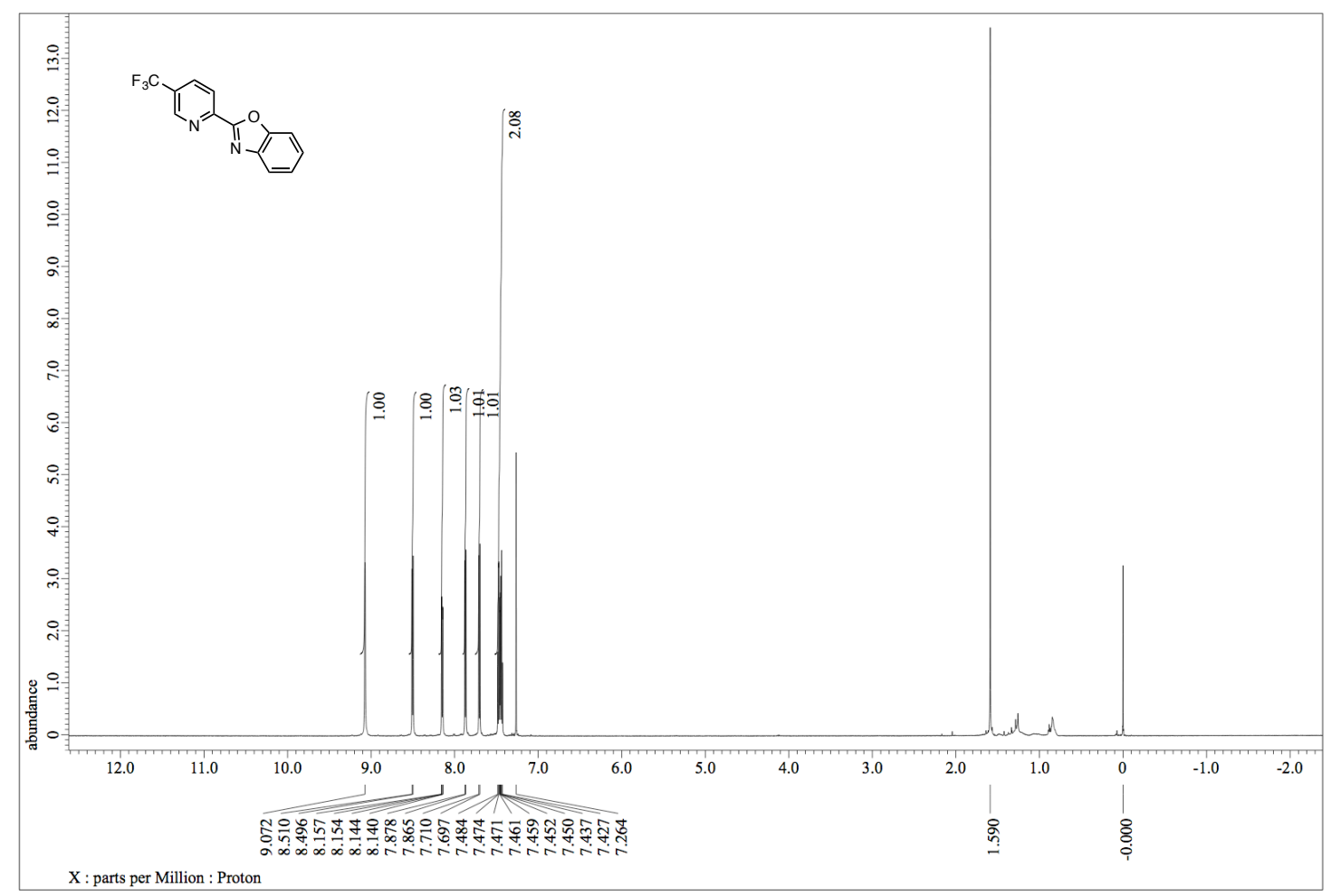

Figure S 41. ${ }^{1} \mathrm{H}$ NMR spectrum of authentic 1h-C6.

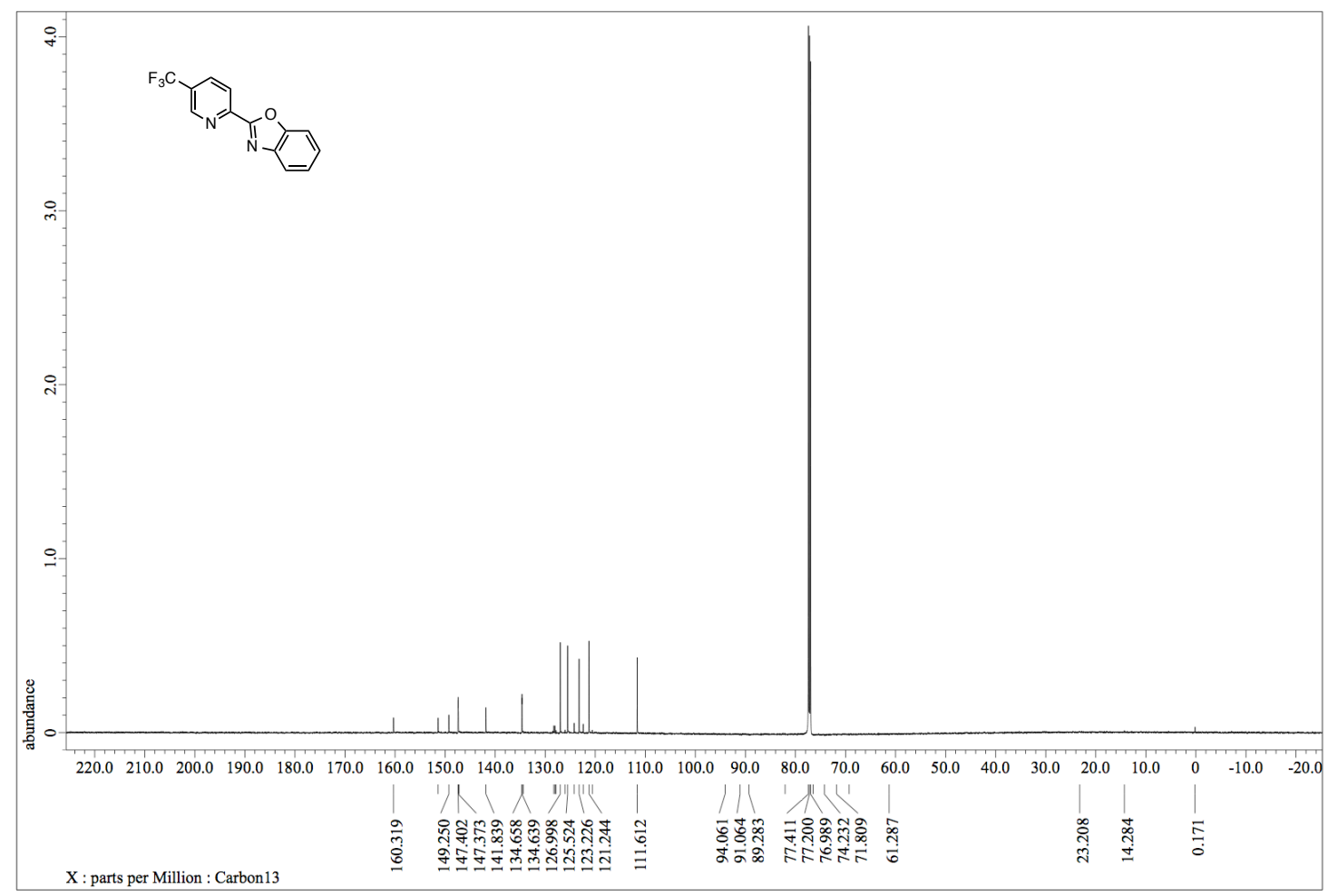

Figure S 42. ${ }^{13} \mathrm{C}$ NMR spectrum of authentic $\mathbf{1 h}-\mathbf{C 6}$. 


\section{Validation of reduction of aryl/benzyl bromide}

Mesityl bromide was reduced to mesitylene during the reaction. Mesitylene was observed by ${ }^{1} \mathrm{H}$ NMR in the crude mixture after evaporation of pyridine.<smiles>c1ccncc1</smiles>

$1.5 \mathrm{~mL}$
$\mathrm{Pd}(\mathrm{OAc})_{2}(10 \mathrm{~mol} \%)$

MesBr (2.0 equiv)

CsOPiv (3.0 equiv)

$170{ }^{\circ} \mathrm{C}, 17 \mathrm{~h}$

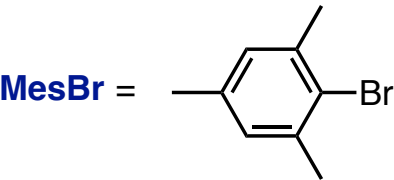<smiles>Cc1cc(C)cc(C)c1</smiles>

1 a $(68 \%$ yield $)$

74\% C3 selectivity (C2/C3/C4 = 19:72:9)

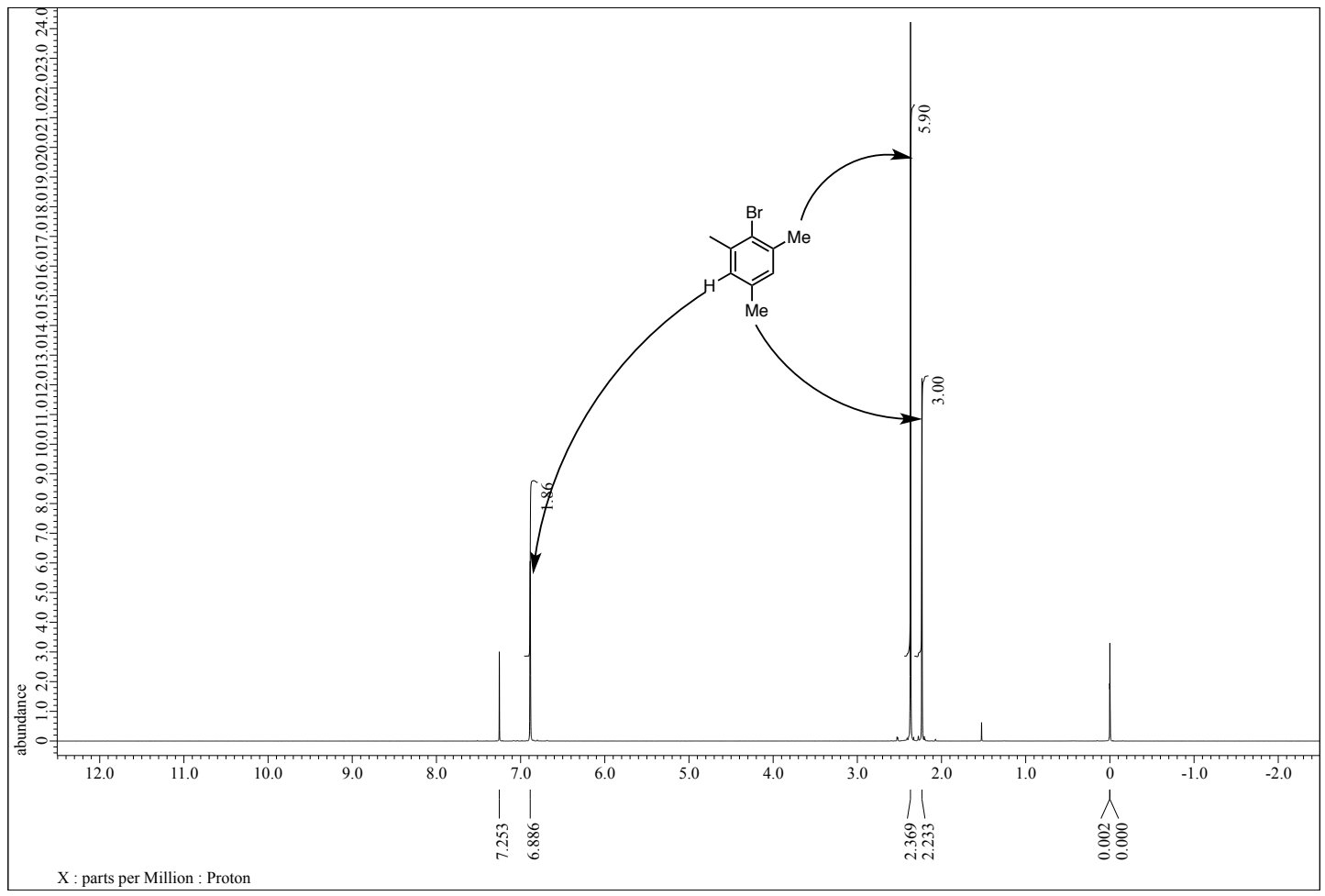

Figure S 43. ${ }^{1} \mathrm{H}$ NMR spectrum of authentic mesityl bromide. 


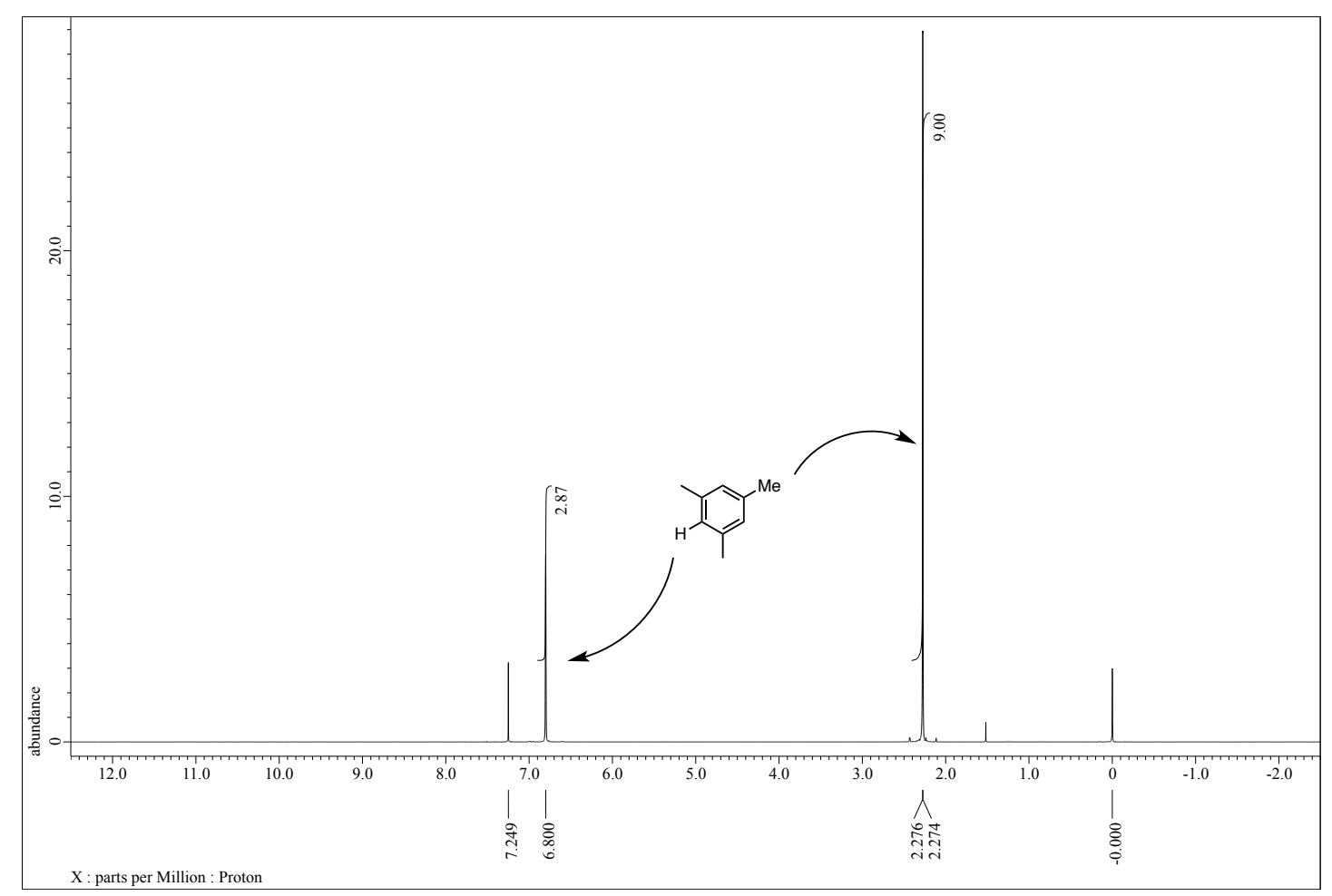

Figure S 44. ${ }^{1} \mathrm{H}$ NMR spectrum of authentic mesitylene.

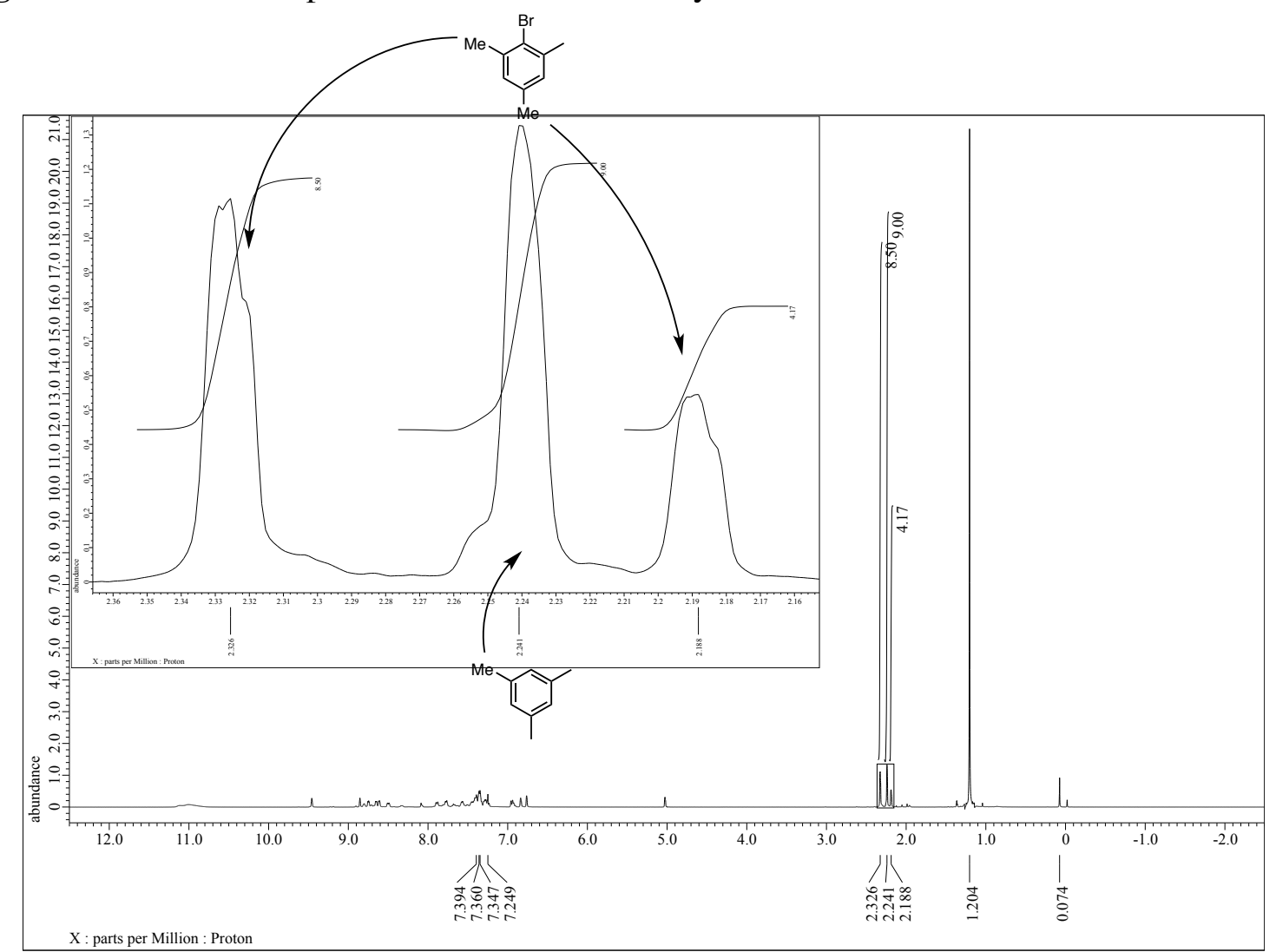

Figure S 45. ${ }^{1} \mathrm{H}$ NMR spectrum of crude reaction mixture of the reaction. 
Benzyl bromide was also reduced to toluene during the reaction. Toluene was detected by GC-MS in the crude mixture.
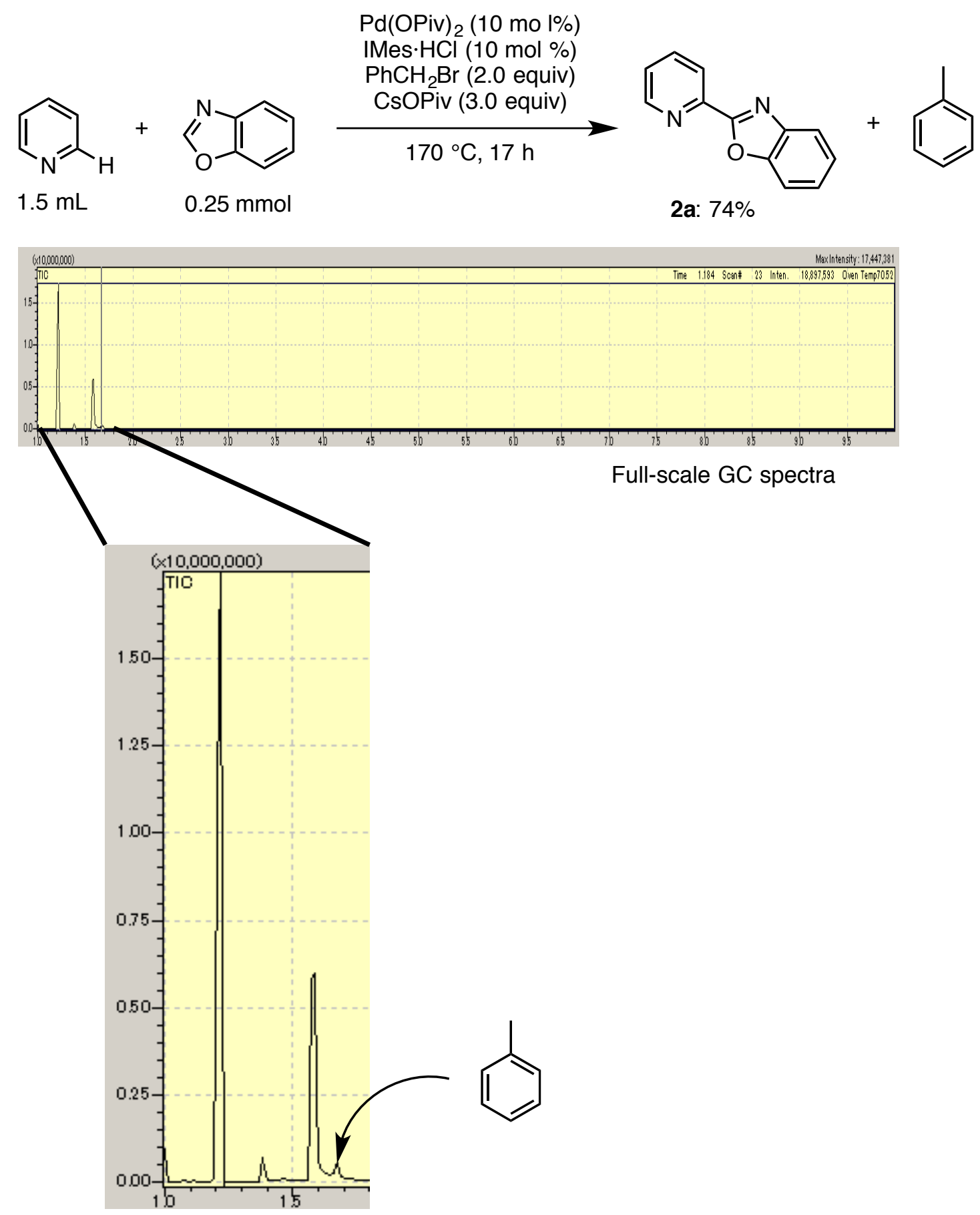

Inset (1.0-1.8 min) GC spectra

Figure S 46. Full-scale and inset (1.0-1.8 $\mathrm{min})$ to GC-MS spectra of the crude mixture. 


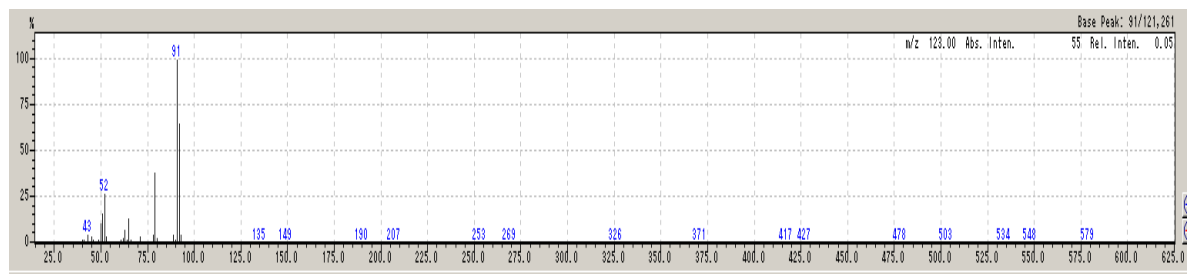

Full-scale MS spectra

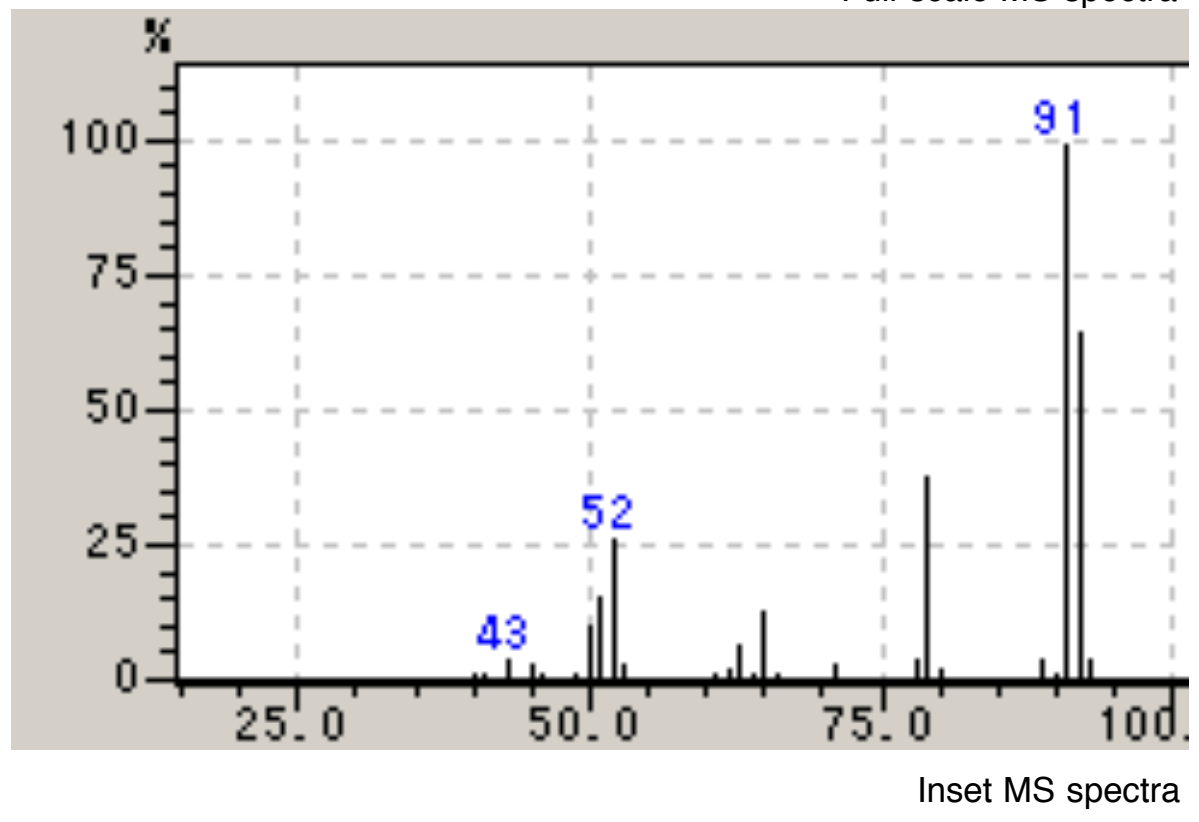

Figure S 47. Full-scale and inset MS spectra at $1.667 \mathrm{~min}$. 